University of Louisville ThinkIR: The University of Louisville's Institutional Repository

Electronic Theses and Dissertations

$5-2016$

\title{
Acrolein is a critical mediator of alcohol-induced liver and intestinal injury in alcoholic liver disease.
}

Wei-Yang (Jeremy) Chen

University of Louisville

Follow this and additional works at: https://ir.library.louisville.edu/etd

Part of the Biochemical Phenomena, Metabolism, and Nutrition Commons, Digestive System Diseases Commons, Medical Cell Biology Commons, Medical Pharmacology Commons, Medical $\underline{\text { Toxicology Commons, and the Translational Medical Research Commons }}$

\section{Recommended Citation}

Chen, Wei-Yang (Jeremy), "Acrolein is a critical mediator of alcohol-induced liver and intestinal injury in alcoholic liver disease." (2016). Electronic Theses and Dissertations. Paper 2477.

https://doi.org/10.18297/etd/2477

This Doctoral Dissertation is brought to you for free and open access by ThinkIR: The University of Louisville's Institutional Repository. It has been accepted for inclusion in Electronic Theses and Dissertations by an authorized administrator of ThinkIR: The University of Louisville's Institutional Repository. This title appears here courtesy of the author, who has retained all other copyrights. For more information, please contact thinkir@louisville.edu. 


\title{
ACROLEIN IS A CRITICAL MEDIATOR OF ALCOHOL-INDUCED LIVER AND INTESTINAL INJURY IN ALCOHOLIC LIVER DISEASE
}

\section{By}

Wei-Yang (Jeremy) Chen

B.S. in Chemistry, Tamkang University, Taiwan, 2003

M.S. in Biochemistry, University of North Carolina-Greensboro, 2009

M.S. in Pharmacology and Toxicology, University of Louisville, 2014

\author{
A Dissertation \\ Submitted to the Faculty of the \\ School of Medicine of the University of Louisville \\ In Partial Fulfillment of the Requirements \\ for the Degree of
}

Doctor of Philosophy in Pharmacology and Toxicology

Department of Pharmacology and Toxicology

University of Louisville

Louisville, Kentucky

May 2016 



\section{ACROLEIN IS A CRITICAL MEDIATOR OF ALCOHOL-INDUCED LIVER AND INTESTINAL INJURY IN ALCOHOLIC LIVER DISEASE}

\section{By}

Wei-Yang (Jeremy) Chen

B.S. in Chemistry, Tamkang University, Taiwan, 2003

M.S. in Biochemistry, University of North Carolina-Greensboro, 2009

M.S. in Pharmacology and Toxicology, University of Louisville, 2014

A Dissertation Approved on

May 19, 2016

By the following Dissertation Committee

Swati Joshi-Barve, Ph.D.

Craig McClain, M.D.

Shirish Barve, Ph.D.

David Hein, Ph.D.

Sanjay Srivastava, Ph.D. 


\section{ACKNOWLEDGMENTS}

I would like to thank my mentors Dr. Swati Joshi-Barve and Dr. Craig McClain, I could not go this far without their full support, dedication in guidance and encouragement throughout my years of research as a graduate student. I would also like to thank Dr. Shirish Barve for his passion in teaching, research advice and encouragement for my project. I would like to thank my graduate committee members: Dr. David Hein, and Dr. Sanjay Srivastava for their guidance, expertise and precious advice in my academic career at the University of Louisville. I would like to express my gratitude to all the members of the McClain/Barve lab for their help and support. And finally, I would like to thank my family in Taiwan for their continuous support, patience and sacrifices in order for me to pursue an excellent opportunity in research and education in the United States and a better future as a scientist. 


\begin{abstract}
ACROLEIN IS A CRITICAL MEDIATOR OF ALCOHOL-INDUCED LIVER AND INTESTINAL INJURY IN ALCOHOLIC LIVER DISEASE

Wei-Yang (Jeremy) Chen
\end{abstract}

May 19, 2016

Alcohol consumption can cause alcoholic liver disease (ALD), which remains a major cause of morbidity and mortality in the United States. Chronic alcohol consumption causes a pro-oxidant environment in the liver and increases hepatic lipid peroxidation. Acrolein is the most reactive and toxic aldehyde generated through lipid peroxidation. Acrolein forms protein adducts and triggers endoplasmic reticulum (ER) stress and hepatocyte apoptosis, which are recognized etiologic factors in ALD. Several studies have established the critical role of the gut-liver axis in ALD pathogenesis, wherein alcohol-induced gut barrier dysfunction contributes to liver injury. This study investigates, in vitro and in vivo, the pathogenic role of acrolein as a major mediator of intestinal barrier dysfunction, and hepatic ER stress and injury in ALD.

Accumulation of acrolein adducts was seen in response to alcohol consumption in mouse livers and intestines. Intestinal acrolein accumulation correlated with serum lipopolysaccharides (LPS), suggesting that elevated 
acrolein is associated with gut permeability. Hepatic acrolein buildup correlated with ER stress, steatosis, JNK activation, apoptosis and liver injury.

Further, hallmark ER chaperones GRP78 and GRP94 were minimally induced, suggesting that ER-adaptive/protective responses were insufficient; the underlying cause was lack of activation of relevant transcription factors. We used cultured hepatic and intestinal cells to examine the direct in vitro effects of acrolein in comparison to alcohol. The in vivo gut-liver effects of alcohol consumption were mimicked by direct in vitro acrolein exposure in intestinal Caco-2 and hepatic H4IIEC cells. Specifically, acrolein down-regulated tight junction proteins, altered their localization, and disrupted barrier function. Similarly, in vitro acrolein exposure in hepatic cells triggered ER stress and induced apoptosis. Notably, these alcohol-induced effects were attenuated by hydralazine, a known acrolein scavenger. 
TABLE OF CONTENTS

PAGE

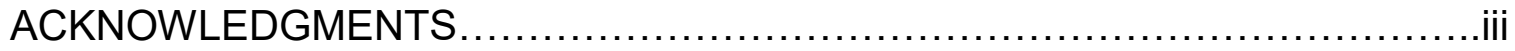

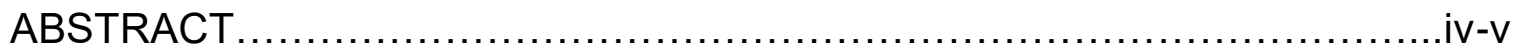

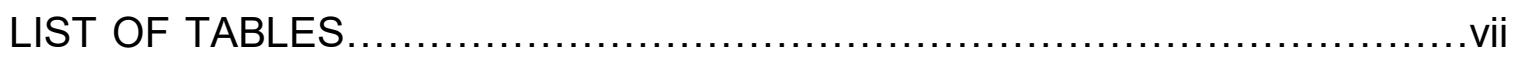

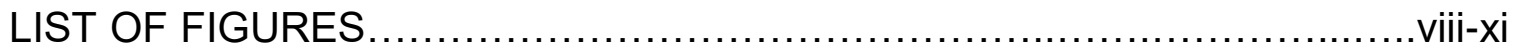

CHAPTER 1: INTRODUCTION..............................................

CHAPTER 2: MATERIALS AND METHODS................................21-27

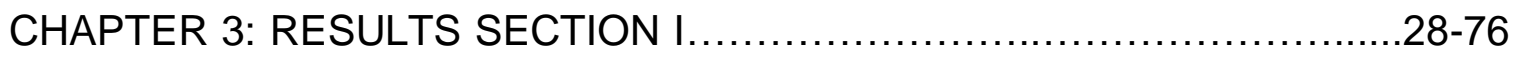

CHAPTER 3: RESULTS SECTION II.........................................77-91

CHAPTER 4: DISCUSSION................................................

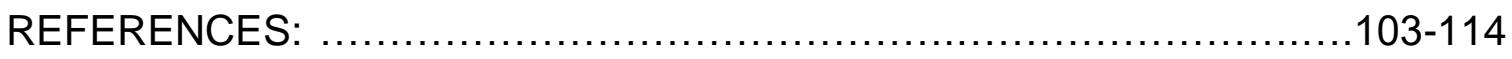

CURRICULUM VITAE .....................................................115-118 


\section{LIST OF TABLES}

$\begin{array}{ll}\text { TABLE PAGE } & \text { PAT }\end{array}$

1. Table 1. Primers for quantitative Real Time-PCR $\ldots \ldots \ldots \ldots \ldots \ldots \ldots \ldots \ldots \ldots \ldots \ldots \ldots \ldots \ldots \ldots \ldots$ 


\section{LIST OF FIGURES}

FIGURE

PAGE

1. Figure 1: Progression of alcoholic liver disease .............................

2. Figure 2. Proposed formation of acrolein from arachidonic acid (PUFA) ........5

3. Figure 3. Chemical structure of acrolein …................................

4. Figure 4. Alcohol metabolism and acrolein detoxification......................14

5. Figure 5. Pathogenesis of the Gut-Liver axis in ALD ........................15

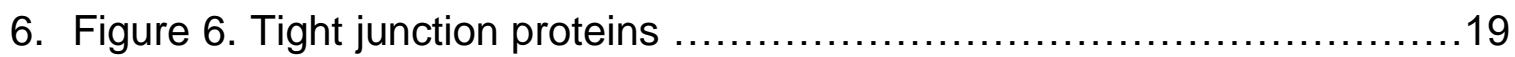

7. Figure 7A. Alcohol consumption leads to accumulation of acrolein-protein adducts in mice livers

8. Figure 7B. Alcohol consumption leads to upregulation of ADH and CYP2E1, and downregulation of GSTP in mice livers

9. Figure 7C. Alcohol consumption leads to downregulation of ADH, CYP2E1, and GSTP in mice livers

10. Figure 8A. Effect of acetaldehyde on alcohol-induced acrolein adduct accumulation in cultured hepatic cells

11. Figure 8B. Effect of pharmacologic modulators of alcohol metabolism on alcohol-induced acrolein adduct accumulation in cultured hepatic cells.......39

12. Figure 8C. Effect of inhibition of GSTP on alcohol-induced acrolein adduct accumulation in cultured hepatic cells. 
13. Figure 8D. Effect of inhibition of GSTP on alcohol-induced acrolein adduct accumulation in cultured hepatic cells.

14. Figure 9A. Alcohol-induced accumulation of acrolein adducts causes hepatic ER stress in mice livers 43

15. Figure 9B. Alcohol-induced accumulation of acrolein adducts causes hepatic ER stress in mice livers .45

16. Figure 9C. Phospho-PERK and phospho-elF2 $\alpha$ protein levels 46

17. Figure 10A. Alcohol-induced accumulation of acrolein adducts induces ER chaperone mRNA expression in mice livers

18. Figure 10B. Alcohol-induced accumulation of acrolein adducts induces minimal ER chaperone proteins in mice livers .50

19. Figure 10C. Alcohol-induced accumulation of acrolein adducts activates ATF6 cleavage and decreases IRE phosphorylation in mice livers

20. Figure 10D. Alcohol-induced accumulation of acrolein adducts does not induce XBP1 splicing in mice livers .52

21. Figure 10E. Alcohol-induced accumulation of acrolein adducts does not induce spliced XBP1 protein in mice livers

22. Figure 11A. Alcohol-induced hepatic acrolein build-up and consequent ER stress leads to proapoptotic signaling in mice livers .56

23. Figure 11B. Alcohol-induced hepatic acrolein build-up and consequent ER stress leads to proapoptotic signaling in mice livers .57

24. Figure 11C. Alcohol-induced hepatic acrolein build-up and consequent ER stress leads to proapoptotic signaling in mice livers 58 
25. Figure 11D. Alcohol-induced hepatic acrolein build-up and consequent ER stress leads to proapoptotic signaling in mice livers

26. Figure 12A. Alcohol-induced acrolein and ER stress leads to steatosis, hepatocyte apoptosis and liver injury in mice livers. .61

27. Figure 12B. Alcohol-induced acrolein and ER stress leads to steatosis, hepatocyte apoptosis and liver injury in mice livers

28. Figure 13A. Acrolein mimics the in vivo effects of alcohol and causes ER stress and cell death in cultured hepatic cells .65

29. Figure 13B. Acrolein mimics the in vivo effects of alcohol and causes ER stress and cell death in cultured hepatic cells .66

30. Figure 13C. ER stress gene expression ATF3, ATF4, GADD/CHOP, GRP78, and GRP94 by alcohol (E) or acrolein (A).

31. Figure 13D. Cell viability of cultured hepatocytes from alcohol $(E)$ or acrolein (A) toxicity. .68

32. Figure 14A. Acrolein scavengers exhibit protective effects in vitro.............71

33. Figure 14B. Acrolein scavengers exhibit protective effects in mice livers......72

34. Figure 14C. Acrolein scavengers exhibit protective effects in mice livers......73

35. Figure 14D. Acrolein scavengers exhibit protective effects in mice livers .....74

36. Figure 14E. Acrolein scavengers exhibit protective effects in mice livers ......75

37. Figure 14F. Acrolein scavengers exhibit protective effects in mice livers ......76

38. Figure 15. Exposure to alcohol causes acrolein adduct buildup in the ileum of

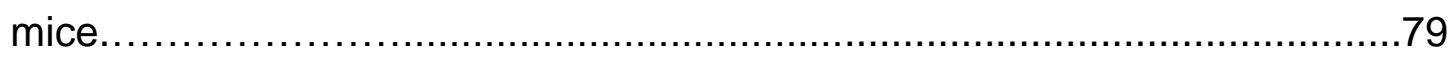

39. Figure 16. Hydralazine attenuated alcohol-induced serum endotoxemia .....80 
40. Figure 17. Exposure to alcohol or acrolein leads to acrolein adduct accumulation in Caco-2 cells monolayers

41. Figure 18. Alcohol exposure decreases barrier function and increases permeability (FD-4) (TEER) Caco-2 cells .84

42. Figure 19. Acrolein exposure decreases and increases permeability (FD-4) barrier function (TEER) in Caco-2 cells .85

43. Figure 20. In vitro alcohol exposure down-regulates TJP mRNAs. (Real time qPCR)

44. Figure 21. In vitro acrolein exposure down-regulates TJP mRNAs. (Real time qPCR) 89

45. Figure 22. Acrolein exposure alters protein levels of TJP....... .90

46. Figure 23. Alcohol treatment causes disrupted distribution of ZO-1 protein in Caco-2. .91

47. Figure 24. Acrolein treatment causes disrupted distribution of ZO-1 protein in Caco-2. 92

48. Figure 25. Schematic: Acrolein mediates alcohol-induced intestinal barrier dysfunction, permeability and hepatic ER stress, apoptosis and injury in ALD, and the scavenger, hydralazine, prevents these effects 102 


\section{CHAPTER 1}

\section{INTRODUCTION}

Alcohol consumption can lead to alcoholic liver disease (ALD), which remains a major cause of morbidity and mortality worldwide and in the United States. Despite the profound economic and health impacts, there is no FDA (Food and Drug Administration) approved therapy for any stage of ALD [1], emphasizing the need for research into therapeutic interventions during the early initiating stages of the disease. Further, only about $20 \%$ of heavy alcohol drinkers develop liver disease, and diet and environment are considered potential determining factors. The pathogenesis of ALD is multi-factorial, and although oxidative stress, lipid peroxidation, and endoplasmic reticulum (ER) stress are known etiologic factors [2], the molecular mediators of hepatic injury remain poorly defined.

\section{Alcohol liver disease (ALD)}

Alcohol consumption is known to cause alcoholic liver disease (ALD) which includes a spectrum of liver disorders (Figure 1), ranging from fatty liver (steatosis) to steatohepatitis, fibrosis, cirrhosis, end stage liver disease and potentially hepatocellular carcinoma (HCC) [3]. Steatosis is commonly found in approximately 90 percent of individuals who consume more than $60 \mathrm{~g}$ per day of alcohol, or about five drinks per day [4]. Steatosis is reversible if the individuals stop or dramatically reduce the amount of alcohol consumption. Histology evidence has shown fat 
accumulation of both small (i.e., microvesicular) and large (i.e., macrovesicular) droplets within liver cells [5]. Some individuals with steatosis progress to more serious liver disease such as alcoholic hepatitis, which is steatosis accompanied by inflammation, neutrophil infiltration, hepatocyte necrosis, and Mallory bodies. Approximately 10 to 35 percent of alcoholic patients may progress from steatosis to fibrosis and eventually cirrhosis followed by end stage liver disease and possibly hepatocellular carcinoma (HCC). The study of mechanisms and mediators that contribute to ALD is critical to understand the development and progression of ALD and to promote therapeutic development.

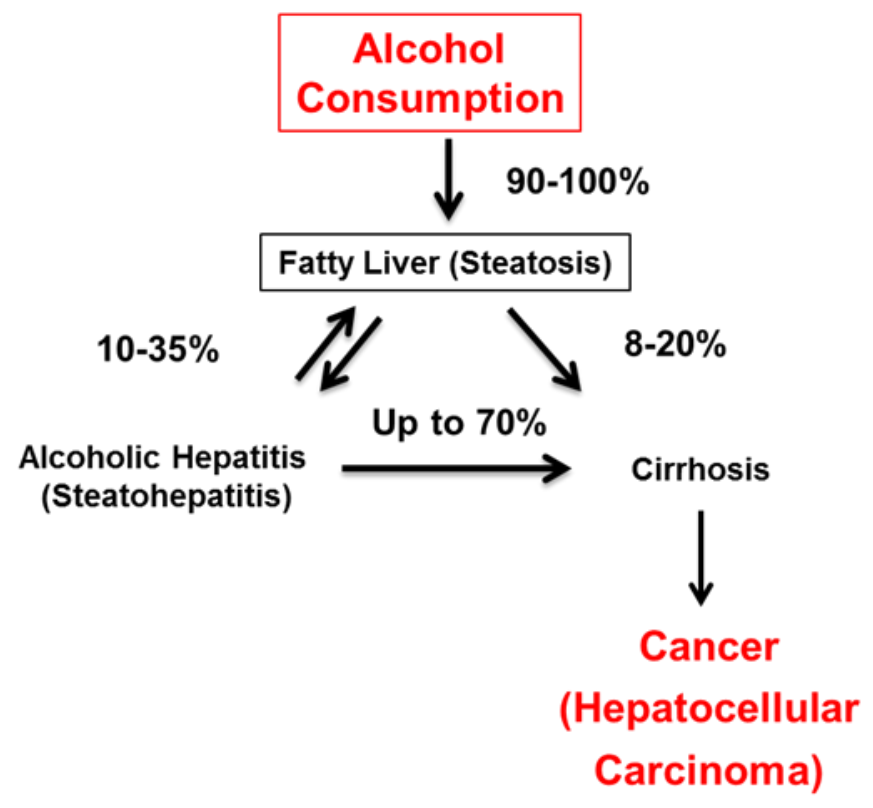

Figure 1: Progression of alcoholic liver disease. 


\section{Alcohol metabolism and oxidative stress}

Ingested alcohol passes through the portal circulation from the intestine and is metabolized primarily in the liver. The process of ethanol metabolism involves at least three distinct enzymatic pathways. Most of the alcohol in the human body is first oxidized in the liver to acetaldehyde which is toxic. This process is catalyzed by the cytosolic alcohol dehydrogenase (ADH), an NAD+-requiring enzyme expressed at high concentrations in hepatocytes. ADH oxidizes ethanol to acetaldehyde, which enters the mitochondria where it is oxidized to acetate by one of several aldehyde dehydrogenases (ALDH) [6]. The microsomal ethanol oxidizing system (MEOS) is the second major pathway for alcohol metabolism and involves the cytochrome P450 enzyme 2E1 (CYP2E1) and the conversion of $\mathrm{NADPH}$ to NADP+. This pathway is known to be highly induced in chronic alcohol drinkers. CYP2E1 also plays an important role in alcohol metabolism; alcohol can be oxidized by CYP2E1 to acetaldehyde, with generation of hydrogen peroxide which causes oxidative stress in the liver cells. Minor pathways for alcohol metabolism involve fatty acid ethyl ester (FAEE) synthase, or catalase in peroxisomes.

Studies have shown that consumption of ethanol disrupts antioxidants function, which leads to elevated oxidative stress and increased interactions between free radicals and cellular components including lipids, proteins, and DNA, which alter cellular structures and functions. The production of reactive oxygen species (ROS) can be compensated for the antioxidant systems of the human body. However, when excessive ROS production exceeds the capacity of 
physiological adaption to eliminate ROS, it could lead to health problems. The superoxide and hydrogen peroxide are the most dominant forms of ROS and they can cause acetaldehyde accumulation due to an imbalance of redox state. Aldehyde dehydrogenase (ADH) is responsible for the oxidation of ethanol by increasing the reduced form of nicotinamide adenine dinucleotide (NADH) which results in a decrease of the $\mathrm{NAD+} / \mathrm{NADH}$ ratio and triggers the conversion of cytosolic xanthine dehydrogenase to xanthine oxidase, which is the enzyme responsible for the production of superoxide radicals. NADH is involved in the conversion of ferritin-bound iron to ferrous (Fe) (II) ions, and these ferrous ions play important roles in free radical reactions which lead to the generation of hydrogen peroxide, hydroxyl radicals, and increased lipid peroxidation [7, 8].

\section{Alcohol-induced lipid peroxidation (LPO) generates Acrolein}

Alcohol metabolism in the liver generates free radicals which cause oxidative degradation of cellular polyunsaturated fatty acids (PUFAs) by LPO. Linoleic acid (LA) is the most common PUFA found in foods in the western diet [9]. The consumption of LA has dramatically increased from $2 \%$ to over $7 \%$ in the last century. Notably, studies by our group and others have shown that consumption of alcohol along with a diet rich in PUFAs (particularly, LA) gives rise to worse alcohol-induced liver injury, compared to alcohol plus saturated fat diet [10]. Moreover, LA is a precursor of arachidonic acid and can be converted to proinflammatory eicosanoids, which are thought to be involved in several chronic diseases, including cancer and cardiovascular disease [11]. Linoleic acid enriched diet is known to elevate lipid peroxidation (LPO). Alcohol-induced LPO is well 
documented in animal models, as well as in patients with ALD. The products of LPO, including malondialdehyde (MDA), 4-hydroxynonenal (HNE) and acrolein, are detected in samples from animals and humans after consuming alcohol. These reactive aldehydes are highly likely to play a critical role in the pathology of ALD. Of particular interest is acrolein which is the most toxic and reactive byproduct of lipid peroxidation [12]. (Figure 2).

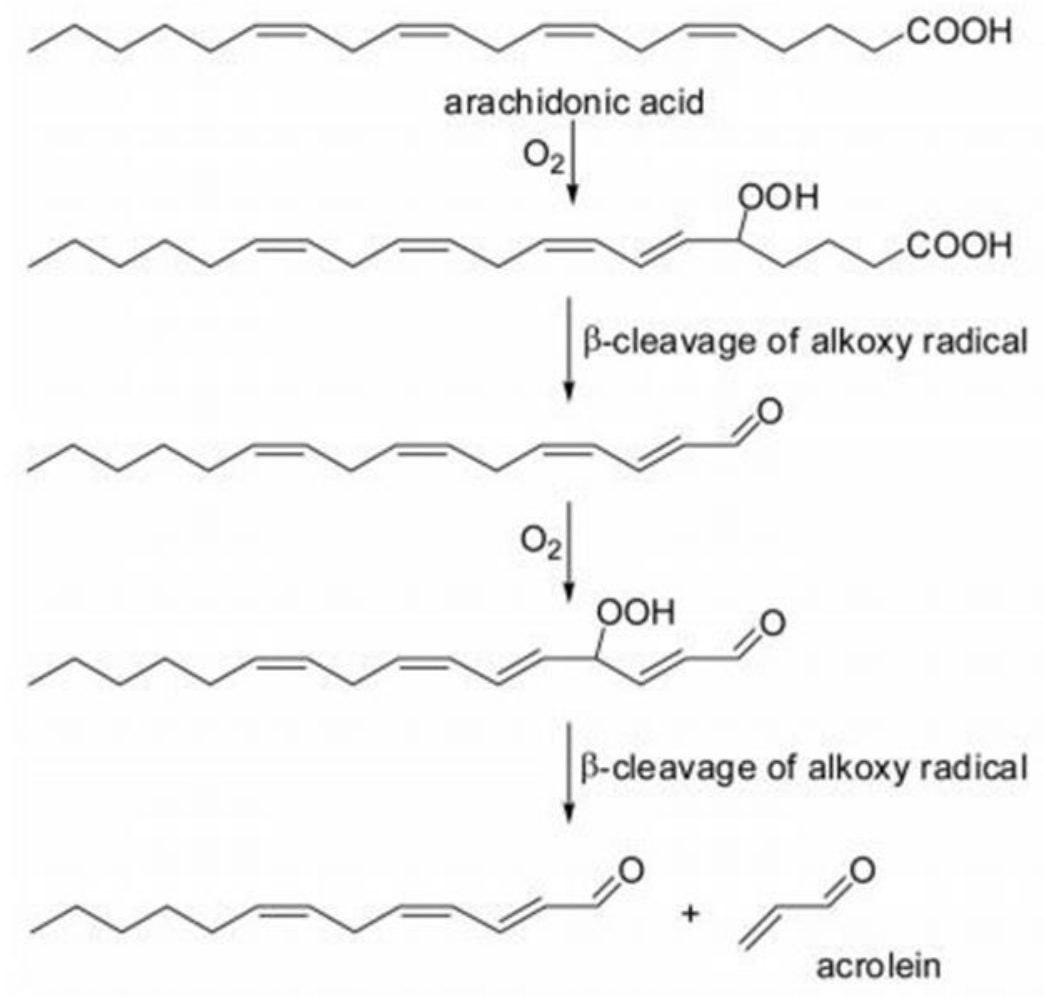

Figure 2: Proposed formation of acrolein from arachidonic acid (PUFA) [13]. 


\section{Acrolein, the reactive aldehyde from lipid peroxidation}

Acrolein is listed as a Harmful and Potentially Harmful Constituent (HPHCs) among volatile carbonyls in tobacco products by Food and Drug Administration (FDA), and inhaled acrolein is a predominant rout of exposure for non-cancer respiratory effects from tobacco smoking. It is a highly reactive $\alpha, \beta$-unsaturated aldehyde with three carbons and a double bond structure (Figure 3) and is formed endogenously during lipid peroxidation [14]. Acrolein is also a biotransformation metabolite formed from certain amino acids, allyl compounds and the anticancer drug, cyclophosphamide [15]. Acrolein has the potential to rapidly deplete cellular glutathione, and it reduces cellular antioxidant capacity [14]. Therefore acrolein may serve as an important oxidative stress biomarker for lipid peroxidation [16]. Acrolein is the most abundant, toxic, and reactive aldehyde among lipid peroxidation derived aldehydes, and it is over 100 times [12] more reactive and forty times greater in concentration than other aldehydes such as 4-HNE [17]. Due to its highly electrophilic nature, acrolein can form adducts with cellular nucleophilic groups in lipids, nucleic acids, and proteins; these acrolein adducts can cause cytotoxicity via irreversible adduction by disrupting cell signaling and mitochondrial function of the cells to produce oxidative stress. Numerous mechanisms of toxicity have been linked to acrolein such as adduct formation, ER stress, oxidative stress, inflammation and mitochondrial dysfunction; acrolein forms covalent adducts with DNA, proteins, and phospholipids. Notably, acrolein is also an environmental pollutant arising from the combustion of wood, paper, fossil fuels, and plastics. It is a major component of cigarette smoke and up to $600 \mu \mathrm{g}$ of acrolein is generated 
per cigarette [18]. This is particularly relevant for ALD given the high prevalence of individuals that use/abuse both alcohol and cigarettes. Additionally, acrolein is produced by overheating fats and oils, and is now considered a significant dietary pollutant occurring in charred meats and fried foods [14, 18]. Thus, both exogenous and endogenous acrolein generation may contribute to the development/progression of liver diseases and the overall level of acrolein may determine the extent of toxic effects and injury.

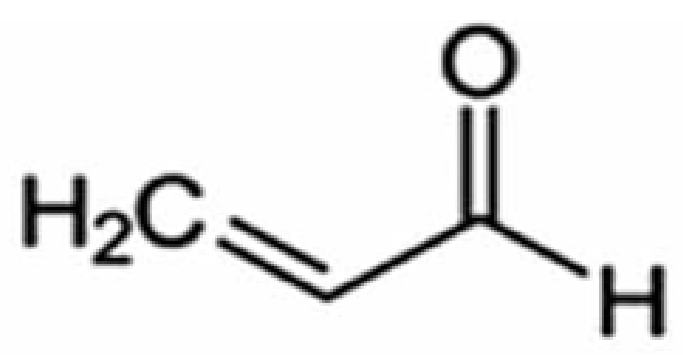

Figure 3: Chemical structure of acrolein.

\section{Acrolein adducts}

Acrolein is a strong electrophile and readily targets critical amino acid residues to form mainly Michael addition-type amino acid adducts with cysteines, histidines and lysines of proteins; the acrolein-lysine adduct, $\mathrm{N} \ll-(3-$ formyl-3,4dehydropiperidino)lysine (FDP-lysine), is readily detectable using specific antibodies [18], and can compromise the function of enzymes, transcription factors, and zinc finger proteins, often with grave consequences. Acrolein is associated with several chronic diseases, and elevated concentrations of acrolein- 
protein adducts are detected in the plasma of patients with several diseases, including Alzheimer's [19], diabetes [20], Parkinson's [21], atherosclerosis [22], chronic obstructive lung disease [14], spinal cord injury, diabetes mellitus, and multiple sclerosis [23]. Accumulation of acrolein-adducted proteins can trigger ER stress and the unfolded protein response [24].

\section{The pathogenic mechanisms of alcohol liver disease}

Some mechanisms postulated to contribute to ALD include inflammation, mitochondrial dysfunction, ER stress, hepatocyte apoptosis and intestinal barrier dysfunction. All of these mechanisms are associated with each other and one mechanism/pathway can lead to other(s).

\section{Inflammation}

Inflammation is an early indicator of more serious liver disease and several inflammatory response pathways are linked to ALD such as NF-kB, JNK, ROS, interleukin-6 (IL-6), and tumor necrosis factor- $\alpha$ (TNF- $\alpha$ ). The inflammatory response is driven primarily by immune and inflammatory cells such as macrophages, monocytes, dendritic cells, neutrophils, and liver-resident Kupffer cells in response to stimuli including LPS, TNFa, etc. The activation of inflammasomes (e.g., NLRP3) also plays an important role in the inflammatory response and results in the production of IL-1 $\beta$, IL-6, IL-18, and TNF- $\alpha$. TNF- $\alpha$ is a cytokine which is responsible for the programmed cell death and apoptosis of hepatocytes in alcohol-induced liver injury. TNF- $\alpha$ also promotes a series of inflammation responses in the hepatocytes and leads to the production of other 
inflammatory cytokines [25]. Persistent cytokine secretion causes chronic inflammation which leads to hepatitis, fibrosis, and cirrhosis [26].

\section{Mitochondrial dysfunction}

Mitochondria are the energy factories of the cells and produce ATP which is required to maintain normal metabolic and repair functions of the cells. Studies have shown that alcohol significantly decreases the viability of hepatocytes due to an inability to maintain a sufficient rate of ATP synthesis. The oxidation of alcohol by ADH and CYP2E1 and acetaldehyde by ALDH leads to a significant increase in the hepatic NADH/NAD+ ratio and an increase in the production of ROS. The enzyme activities of NADH dehydrogenase, succinate dehydrogenase and ATP synthase are depressed when exposed to reactive species, including superoxide, hydrogen peroxide and peroxynitrite, leading to the inactivation of mitochondrial proteins and further decreases in the function of mitochondria in the cells $[27,28]$. Disruption of mitochondrial function can trigger signaling cascades involving the release of pro-apoptotic proteins from mitochondria, and activation of initiator and effector caspases, leading to apoptotic cell death.

\section{Endoplasmic Reticulum (ER) stress and unfolded protein response (UPR)}

The endoplasmic reticulum (ER) is a network of interconnected tubules within the cytoplasm of eukaryotic cells [29]. The ER plays a crucial role in cell homeostasis and survival, and is responsible for synthesis, folding, assembly, and trafficking of proteins; lipid biosynthesis; and regulation of intracellular calcium and redox homeostasis [30]. The molecular chaperones/folding proteins in the ER (e.g., Grp78/BiP, Grp94, protein disulfide isomerase (PDI), calnexin, and calreticulin) 
ensure that only properly folded proteins reach their destinations; inappropriately folded proteins are retained in the ER lumen for refolding or endoplasmic reticulum-associated degradation (ERAD) [30]. Certain stimuli (such as altered redox status, oxidative stress, unbalanced calcium, hypoxia, or energy deprivation) can disturb ER function and lead to the accumulation of unfolded proteins in the ER, thereby triggering ER stress and the unfolded protein response (UPR). To restore ER homeostasis, the UPR adaptive mechanism upregulates the folding capacity of the ER through induction of chaperones and foldases, and downregulates the biosynthetic load through inhibition of protein synthesis and degradation of misfolded proteins [30].

ER stress/UPR signaling pathways involve three major ER resident transmembrane sensor proteins, inositol requiring protein 1 (IRE1), ds-RNAactivated protein kinase (PKR) like ER kinase (PERK), and activating transcription factor 6 (ATF6), which are activated from their inhibitory binding with chaperone GRP78/BiP. The first sensor, IRE1, a type I transmembrane protein with both a serine/threonine kinase domain and endoribonuclease (RNase) activities, is activated by transautophosphorylation. IRE1 transcriptionally forms spliced XBP1 (sXBP1) which activates UPR target genes and ERAD pathway genes. IRE1 also activates the stress-signaling kinase, $\mathrm{JNK}$, and the transcription factor, $\mathrm{NFKB}$, both of which can lead to proinflammatory cytokine production. The second sensor, PERK, a type I transmembrane protein with a cytosolic serine/threonine kinase domain, phosphorylates the eukaryotic initiation factor $2 \alpha$-subunit (elF2 $2 \alpha$ ), leading to inhibition of translation and reduction of the protein load during ER stress. Also, 
p-elF2 $\alpha$ triggers activating transcription factor 4 (ATF4), which regulates ER chaperone genes, ERAD pathway genes, amino acid metabolism genes, and the proapoptotic transcription factor C/EBP homologous protein (CHOP, GADD153). The third sensor, ATF6, a type II ER transmembrane protein with a CREB/ATF bZIP transcription factor domain at the amino terminus, is activated by cleavage in the Golgi complex and can translocate to the nucleus to activate chaperone gene expression [31, 32]. Additionally, ATF6 also leads to upregulation of CHOP, and affects SREBP activity and consequently, lipogenesis.

\section{ER stress induces apoptosis}

In order to counter ER stress in the cells, the UPR adaptive signaling pathway is triggered. However, excessive or prolonged ER stress can overwhelm the adaptive pathways, and cause apoptotic and necrotic cell death. The apoptosis pathway is triggered to protect the cells if the PERK, ATF6, and IRE1 adaptive pathways fail to mitigate ER stress [33]. Several mechanisms are involved in ER stress-induced apoptosis such as the transcription factor C/EBP homologous protein (CHOP), JNK, Bcl-2 family proteins, calcium and redox homeostasis, and caspase activation [12].

ER stress triggers the JNK pathway via phosphorylation of IRE-1 with the adaptor protein TRAF2 (tumor necrosis factor receptor (TNFR)-associated factor2) which phosphorylates and activates JNK [34]. Phosphorylated JNK activates proapoptotic Bim and inactivates antiapoptotic $\mathrm{Bcl}-2$ proteins, and triggers release of cytochrome $C$ through the mitochondrial death pathway. Calcium homeostasis is associated with apoptosis; excess calcium release from the ER results in 
calcium accumulation in the mitochondria and results in release of apoptosis effectors from mitochondria into the cytosol. Bcl-2 has the ability to reduce the overflow of free calcium stored in the ER and prevent cell apoptosis.

The transcription factor CHOP/GADD153 is the best-known proapoptotic pathway associated with ER stress. CHOP is a bZIP-containing transcription factor that is a common point of convergence for all three arms of the UPR, with binding sites for ATF6, ATF4 and XBP1s present within its promoter. CHOP is primarily considered a pro-apoptotic transcription factor that mediates ER stress induced cell death through the regulation of $\mathrm{Bcl}-2$ family members. Also, $\mathrm{CHOP}$ transcriptionally induces GADD34 and dephosphorylates $p$-elF2 $\alpha$ to enable protein synthesis, concomitantly generating ROS and leading to oxidative stress and inflammation [12]. The activation of ER stress, and consequent inflammation and apoptosis may be a significant contributor to many forms of liver injury, including ALD.

ER stress has been reported to contribute to alcohol-induced liver injury and the progression of alcoholic liver disease in both animal and cell models [35-38]. Upregulation of ER stress related genes such as Grp78, Grp94, and CHOP has been observed in acute and chronic alcohol feeding animal models and in patient biopsies $[39,40]$. Some factors that are thought to contribute to alcohol-induced ER stress include acetaldehyde, oxidative stress, disrupted calcium homeostasis, impaired one carbon metabolism, and epigenetic modifications. 


\section{Metabolism and removal of acrolein and scavengers}

Acrolein can deplete intracellular glutathione (GSH), GSH-linked glutathione-Stransferases, and aldose reductase rapidly for its detoxification [41], and acrolein can be toxic unless removed or metabolized [42]. The primary pathway of acrolein metabolism is conjugation with glutathione catalyzed by glutathione S-transferaseP (GSTP) [43, 44]. Acrolein is also metabolized by aldehyde dehydrogenase (ALDH2), which is primarily responsible for clearance of acetaldehyde in the alcohol metabolism. A recent study has shown that GSH is a major acroleindetoxifying compound. In the brain infarction of aging there is an increase in acrolein and a decrease in GSH [45] (Figure 4). Acrolein scavengers have been used clinically for other conditions and have demonstrated protection against aldehyde-induced injury in experimental disease models. Hydralazine, a vasodilator and hypertension drug, was chosen due to it is effectiveness in preventing acrolein-mediated oxidative stress and cell death in PC12 cells, and tissue injury in spinal cord injury models [46]. Notably, the cytoprotective effects of hydralazine are shown to occur not merely by acrolein scavenging but also by effective trapping of acrolein-protein adducts [47]. Another acrolein scavenger, carnosine, is a dipeptide and inhibits acrolein from forming protein-protein crosslinking and forming protein carbonyl groups [48]. 


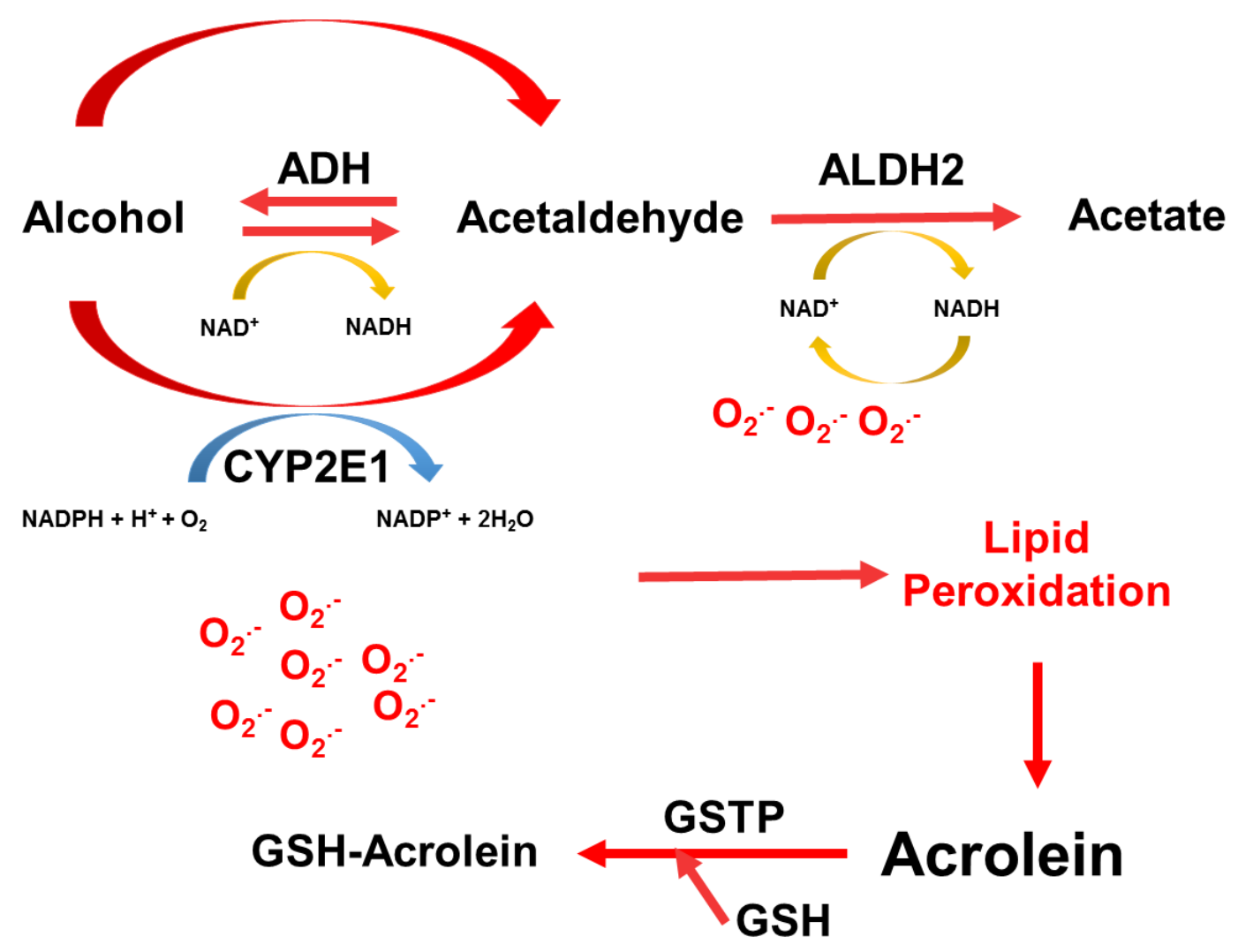

Figure 4: Alcohol metabolism and acrolein detoxification.

\section{Gut-liver axis in alcoholic liver disease}

Alcohol quickly distributes into the portal circulation and across biological membranes to organs throughout the body. Alcohol-induced injuries are observed in many organs including brain, immune system, kidney, lung, heart, pancreas, and particularly, the gastrointestinal tract and liver. Increasing evidence suggests that interactions between the intestine and liver (gut-liver axis) play a critical role in the development/progression of ALD [49]. Alcohol is known to disrupt intestinal epithelial barrier function and increase intestinal permeability, allowing translocation of bacteria and bacterial products such as endotoxin, flagellin, etc. Endotoxin (lipopolysachharide, LPS) is recognized by toll-like receptors (TLRs) on 
liver Kupffer cells (and possibly hepatocytes), resulting in inflammation and release of proinflammatory cytokines such as TNFa, IL-1 $\beta$, IL-8 and IL-18. These cytokines, in turn, can affect both the liver and the intestine, and promote further injury. In the liver, alcohol is known to cause ER stress, inflammation and hepatocyte apoptosis and injury. Thus, interactions of the intestine and liver involving altered intestinal permeability and systemic bacterial translocation, as well as hepatic ER stress, inflammation and injury are critical in the development and progression of ALD (Figure 5).

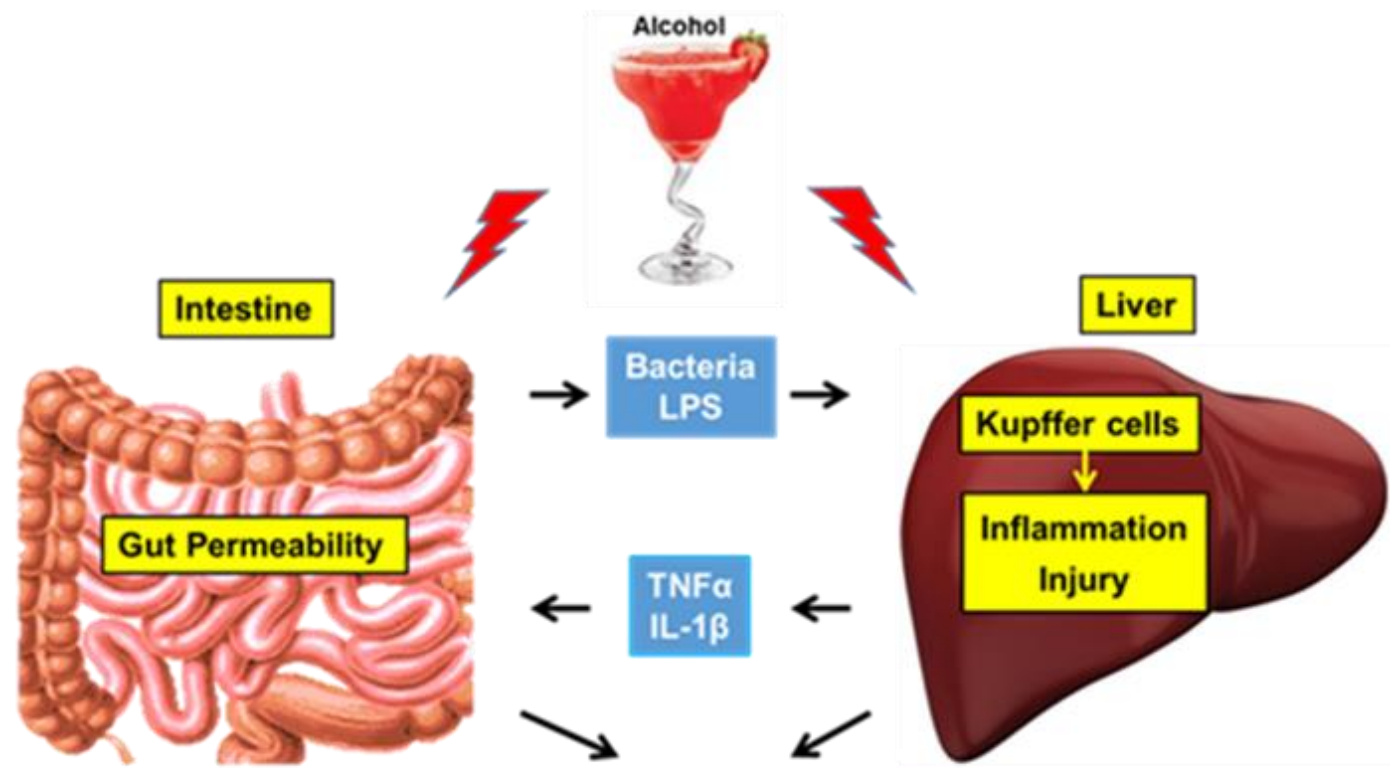

Alcoholic Liver Disease

Figure 5: Pathogenesis of the Gut-Liver axis in ALD.

\section{Intestinal barrier dysfunction and intestinal permeability}

Intestinal barrier dysfunction is linked to various intestinal disorders including inflammatory bowel disease, celiac disease, and diarrheal infection [50]. Several disorders such as multiple sclerosis, Alzheimer's, Parkinson's and autism are 
linked to intestinal barrier dysfunction and involve the activation of the inflammatory response due to altered intestinal permeability (or leaky intestine) [51]. Importantly, altered intestinal permeability is considered as a major causal factor in the pathology of ALD [52]. Intestinal barrier function is maintained by several components: mucus layer, anti-microbial peptides, tight junctions, and adherence junctions. The tight junctions are controlled by the tight junction proteins, including ZO proteins, claudins, and occludins [53]. The pathogenesis of several gastrointestinal diseases relies mostly on disruption of tight junction proteins $[54,55]$. The intestinal barrier is primarily maintained by a single layer of epithelial cells formed between the lumen and mucosal tissues which play a critical role in maintaining normal human biological function [56]. It is the key barrier in the body to prevent invasion of toxins, antigens and microorganisms and loss of water and electrolytes. Moreover, it facilitates digestion and absorption of nutrients from the diet $[53,57]$. The intestinal epithelium is comprised of several different cell types organized into crypts and villi. The cellular components of the intestinal barrier includes the vascular endothelium, the epithelial cell lining, and the mucus layer [53]. The principal cell types consist of paneth cells, goblet cells, enterocytes, and enteroendocrine cells [58]. Among these cells, the paneth cells in the crypts of the small intestine produce digestive secretions, immune molecules, cell products such as cytokines, inflammatory mediators and antimicrobial peptides (small peptides which can function as chemoattractants), dendritic cell activators, and direct antimicrobial effectors $[53,59]$. Intestinal permeability is closely associated with the intestinal 
commensal microbiota and the innate immune system. The alterations of intestinal permeability include gut microbiota modifications, mucus layer alterations, and epithelial damage, which allow translocation of luminal contents to the inner layers of the intestinal wall [53] or the circulation.

\section{Tight junction proteins}

All intestinal epithelial cells are connected at lateral membranes through formation of an impermeable seal to the paracellular space with three major types of junctional complexes--tight junctions, adherens junctions, and desmosomes. These junctional complexes maintain intestinal barrier function and cell polarity by separating the apical from basolateral membranes. Among these major junctional complexes, the tight junctions are controlled by the tight junction proteins (TJPs) which include claudins, occludin, zonula occludens occludins (ZO), junctional adhesion molecules (JAM), and tricellulin proteins (Figure 6). The adherens junctions are located below tight junctions and are important in cell-cell signaling, epithelial restitution. Desmosomes support epithelial stability [56]. TJPs are multiprotein complexes located at the most apical end of the lateral membrane and are composed of both transmembrane and cytoplasmic plaque proteins that interact with the cytoskeleton directly. Moreover, TJs are the critical element of mucosal epithelial permeability because of their ability to selectively control the paracellular permeability for small molecules, ion, and water [57]. Therefore, the expression of TJPs determines the normal function of the intestinal barrier and alterations are associated with changes of intestinal permeability. 
The intercellular adhesion and paracelluar permeability are maintained and regulated by the interactions of these TJPs in the cell surface [60]. Among these key TJPs, claudins, occludin, junctional adhesion molecules (JAM), and tricellulin are the major transmembrane proteins for establishing the selective paracellular barrier. Intracellular molecules such as zonula occludens (ZO) and cingulin are the major cytoplasmic plaque proteins located at the peripheral membrane [61]. In the intestinal barrier structure, occludin, claudins, and tricellulin proteins are linked to the adjacent cells in the actin cytoskeleton through cytoplasmatic scaffolding proteins like ZO proteins [56]. Claudins are the backbone for constructing the tight junction structure [62]. Interestingly, in the different segments of the GI tract, each claudin shows a unique and diverse tissue expression pattern [57]. The ZO proteins include three members (ZO-1, ZO-2, and ZO-3) [63], and the JAM family includes three classical members (JAM-1, -2, and -3) and four related molecules (JAM-4, JAM-L, CAR, and ESAM) [64]. Alterations in the levels and/or the subcellular localization of these TJPs can result in intestinal barrier dysfunction. Thus, TJPs are critical components of intestinal barrier function. 


\section{Epithelial Tight Junctions}

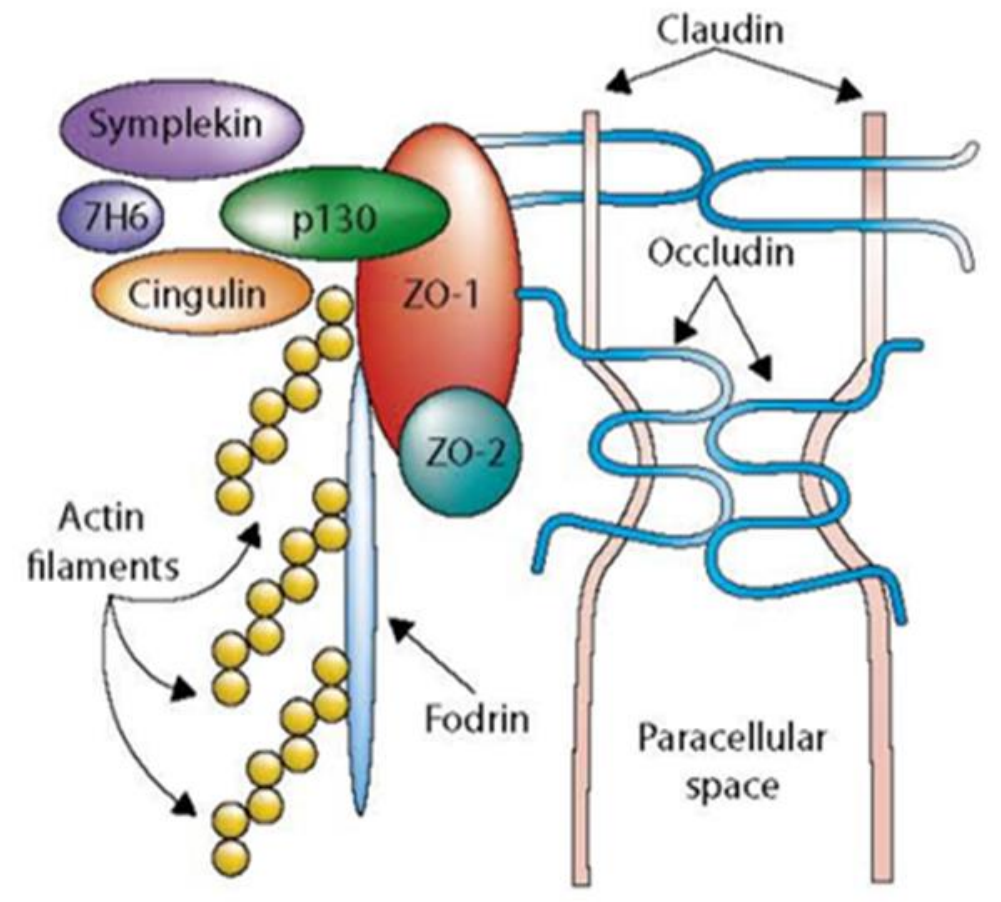

Figure 6: Tight junction proteins [65]. 


\section{HYPOTHESIS}

We hypothesize that acrolein plays a pathogenic role in the gut-liver injury of ALD. Specifically, our hypotheses are:

1) Acrolein generation/accumulation in the liver contributes to alcohol-induced liver injury by causing ER stress and consequent apoptotic cell death of hepatocytes.

2) Acrolein generation/accumulation in the intestine causes intestinal epithelial barrier dysfunction leading to increased gut permeability and systemic bacterial translocation by disruption of tight junctions, induction of ER stress and epithelial cell death/dysfunction.

3) Acrolein clearance/removal protects against alcohol-induced gut-liver injury in ALD. Acrolein scavengers (hydralazine) will alleviate accumulation of acrolein on alcohol-induced intestine and liver injury in ALD.

\section{SPECIFIC AIMS}

Aim 1: To determine the impact of acrolein in alcohol-induced liver ER stress, apoptosis and injury in ALD.

Aim 2: To determine the therapeutic potential of acrolein scavengers in protection against alcohol-induced liver injury in ALD.

Aim 3: To determine the role of acrolein in alcohol-induced intestinal dysfunction in ALD. 


\section{CHAPTER 2 \\ MATERIALS AND METHODS}

\section{Animal studies}

Male C57BL/6J mice (10 weeks of age) were obtained from Jackson laboratories (Bar Harbor, ME). They were maintained at $24^{\circ} \mathrm{C}$ with a $12 \mathrm{~h}: 12 \mathrm{~h}$ light/dark cycle and had free access to normal chow diet and tap water for 5 days before the start of the experiment. The mice were fed (ad libitum) a Lieber-DeCarli liquid diet (BioServ Inc., Frenchtown, $\mathrm{NJ}$ ) containing $5 \%$ ethanol (w/v or $35 \%$ of calories) or maltose dextrin as control for 10 days, followed by a single oral gavage of ethanol (5g/kg body weight) on day 11 . After 9 hours, the mice were anesthetized with Avertin. Plasma and tissue samples were collected for assays. Part of the liver from the left lobe was harvested and fixed in 10\% neutral-buffered formalin, while the remaining liver tissue was snap frozen in liquid $\mathrm{N}_{2}$ and stored at $-80^{\circ} \mathrm{C}$. All mice were treated according to the protocols reviewed and approved by the Institutional Animal Care and Use Committee of the University of Louisville.

\section{Reagents}

General chemicals, carnosine, hydralazine, acrolein, and $\beta$-actin antibody were purchased from Sigma Aldrich (St. Louis, MO). All other antibodies were purchased from Cell Signaling (Beverly, MA). Cell culture supplies were obtained from Invitrogen (Carlsbad, CA).

\section{Cell culture}


H4IIEC, a rat hepatoma cell line was obtained from American Type Culture Collection (Rockville, MD). All treatments were performed on sub-confluent monolayers of cells. Cells were plated at the following densities: (i) 20,000 cells per well for 96 -well plates; (ii) $0.25 \times 10^{6}$ cells per well for 24 -well plates; (iii) $0.5 \times 10^{6}$ cells per well for 6 -well plates; (iv) $2.5 \times 10^{6}$ cells per $100 \mathrm{~mm}$ plates. Cells were cultured in the DMEM media ( $10 \%$ horse serum, $5 \%$ fetal bovine serum) at $37^{\circ} \mathrm{C}$ in a $5 \% \mathrm{CO}_{2}$ environment. Cells were counted using a hemocytometer.

Caco-2 cells are colon carcinoma cells, which undergo a process of spontaneous differentiation in culture and fully differentiate into mature enterocytes in 21 days [66]. Caco-2 cells were obtained from the ATCC and were cultured in Eagle's minimal essential medium supplemented with $100 \mathrm{U} / \mathrm{mL}$ penicillin, 100 $\mu \mathrm{g} / \mathrm{mL}$ streptomycin, and $10 \%$ fetal bovine serum at $37{ }^{\circ} \mathrm{C}$ in a $5 \% \mathrm{CO}_{2}$ environment.

\section{Caco-2 monolayer barrier function analysis}

Resistance (TEER)

Caco-2 cells were cultured in Transwell inserts (Costar, Corning) for 21 days to polarize and differentiate into an epithelial cell-like monolayer. Caco-2 monolayers were measured with an epithelial volt ohmmeter (World Precision Instruments, Sarasota, FL). Electrical resistance was recorded with three consecutive measurements after subtracting the resistance value of the filters alone.

FITC-Dextran Assay (FD4)

Caco-2 cells were cultured in transwell inserts (Costar, Corning) for 21 days to polarize and differentiate into epithelial cell-like monolayer. Fluorescein 
isothiocyanate-dextran-4 (FD-4) was added to the apical top of the culture and incubated for $30 \mathrm{~min}$. The basal medium was collected for Caco-2 cell permeability measurement using a microplate fluorescence reader with an excitation wavelength of $485 \mathrm{~nm}$ and an emission wavelength of $530 \mathrm{~nm}$.

\section{Isolation of RNA and RT-PCR}

The messenger RNA (mRNA) levels of ER stress genes were analyzed by real time polymerase chain reaction (RT-PCR). Total RNA (300ng), isolated using Trizol (Invitrogen, Carlsbad, CA), was used in reverse transcription reactions as described by the manufacturer. The resulting complementary DNA was then subjected to PCR using specific primer pairs (Table 1), with 18s and TATA-binding protein as internal controls. SYBR Green I dye was used for Real Time PCR in an $A B I$ prism 7500 sequence detection system.

\section{Small interfering RNA transfection}

Prior to alcohol or acrolein treatment, siRNAs specific for rat GSTP or scrambled RNA (Thermo Fisher Scientific) as a negative control were transfected into H4IIEC cells with Lipofectamine® RNAiMAX Transfection Reagent (Thermo Fisher Scientific) according to the manufacturer's instructions. The final concentration of each transfected RNA was $25 \mathrm{nM}$. Inhibition of GSTP was confirmed by examining GSTP mRNA and protein. Cells were treated $72 \mathrm{~h}$ after transfection with alcohol or acrolein for $24 \mathrm{~h}$. 


\begin{tabular}{|l|l|}
\hline Mouse & \\
\hline mATF3_F1 & GCGCTGGAGTCAGTTACCGTCA \\
\hline mATF3_R1 & TTCTTCAGGGGCCGCCTCAGAC \\
\hline mATF4_F1 & AAGCCATGGCGCTCTTCACGA \\
\hline mATF4_R1 & AGTCCCCCGCCAACACTTCG \\
\hline mDDIT3_GADD_CHOP_F1 & CCGGAACCTGAGGAGAGAGTGTT \\
\hline mDDIT3_GADD_CHOP_R1 & AGCTGCCATGACTGCACGTGG \\
\hline mGRP78_F1 & ACCACCTATTCCTGCGTCGGTGT \\
\hline mGRP78_R1 & AGGCCACATACGACGGCGTG \\
\hline mGRP94_F1 & AAAGGACTTGCGACTCGCCGG \\
\hline mGRP94_R1 & TCTGACGAACCCGAAGGTCAGC \\
\hline mCyp2e1_F & AGGGGACATTCCTGTGTTCC \\
\hline mCyp2e1_R & TTACCCTGTTTCCCCATTCC \\
\hline mGstp1_F1 & CCCTCTGTCTACGCAGCACT \\
\hline mGstp1_R1 & CTCACACCGCCCTCGAAC \\
\hline mAdh1_F & TGTTGAGAGCGTTGGAGAAG \\
\hline mAdh1_R & CGCTTCGGCTACAAAAGTTG \\
\hline Rat & \\
\hline rATF3_F1 & GGGCCACCTCAGACTTGGTGACT \\
\hline rATF3_R1 & CATCGGATGTCCTCTGCGCTGG \\
\hline rATF4_F1 & GACAAGGCGGGCTCCTCAGAA \\
\hline rATF4_R1 & AAAGGCATCCTCCTTGCCGGTGT \\
\hline rDDIT3_GADD_CHOP_F1 & TGTTGAAGATGAGCGGGTGGCAG \\
\hline rDDIT3_GADD_CHOP_R1 & TGGACCGGTTTCTCTCTCCTCAGGT \\
\hline rGRP78_F1 & GGACCACCTATTCCTGCGTCGGT \\
\hline rGRP78_R1 & TGGCCGCATCGCCAATCAGA \\
\hline rGRP94_F1 & CGTCCTGCTGACCTTCGGGTTT \\
\hline rGRP94_R1 & CCAGGTCCTCTTCTACCGTGCCA \\
\hline Human & \\
\hline hsTj1_F (ZO-1) & AGCACAGCAATGGAGGAAAC \\
\hline hsTjp1_R (ZO-1) & CCCCACTCTGAAAATGAGGA \\
\hline hsCldn1_F & GGCAGATCCAGTGCAAAGTC \\
\hline hsCldn1_R & GGTGGCCACAAAGATTGCTA \\
\hline hsOcln_F & ATGCCCTCTGCAACCAATTT \\
\hline hsOcln_R & CCACAATAATCATGAACCCCAGT \\
\hline Human/Rat/Mouse & \\
\hline 18Sreg3F & CTCAACACGGGAAACCTCAC \\
\hline 18Sreg3R & CGCTCCACCAACTAAGAACG \\
\hline
\end{tabular}

Table 1: Primers for quantitative Real Time-PCR. 


\section{MTT assay}

Cell viability was assessed by the 3-(4,5-dimethythiazol-2-yl)-2,5diphenyltetrazolium bromide (MTT) assay. After treatments, cells were washed twice with PBS; then cell culture medium was replaced with medium containing 1 $\mathrm{mg} / \mathrm{ml} \mathrm{MTT}$. After $1 \mathrm{~h}$, cells were lysed with $100 \mathrm{ul}$ lysis buffer containing $20 \%$ SDS and $50 \% \mathrm{~N}, \mathrm{~N}$-dimethyformamide (DMF), and incubated at $37^{\circ} \mathrm{C}$ overnight. The OD values were read at $570 \mathrm{~nm}$.

\section{Western blot analysis}

Cells were lysed in lysis buffer $(50 \mathrm{mM}$ Tris $\bullet \mathrm{HCl}, \mathrm{pH} 7.4,150 \mathrm{mM} \mathrm{NaCl}, 2 \mathrm{mM}$ EDTA, 4 mM Na3VO4, 40 mM NaF, 1\% Triton X-100, 1 mM PMSF, 1\% protease inhibitor cocktail) and centrifuged at $14,000 \mathrm{~g}$ for $10 \mathrm{~min}$. The supernatants were collected and $26 \mu \mathrm{g}$ of equivalent protein in total cell lysates was resolved by SDSpolyacrylamide gel electrophoresis and transferred onto a polyvinylidene difluoride membrane (Bio-Rad, Hercules, CA). Membranes were blocked for $1 \mathrm{~h}$ in blocking buffer $(5 \%$ nonfat dry milk in 0.1\%TBST (10 mM Tris-HCl, pH 8.0, $150 \mathrm{mM} \mathrm{NaCl}$, and $0.1 \%$ Tween 20)) and incubated overnight at $4{ }^{\circ} \mathrm{C}$ with the primary antibodies diluted in blocking buffer. After washing with $0.1 \%$ TBST, the membranes were incubated with appropriate secondary antibodies for $1 \mathrm{~h}$ at room temperature. Proteins were visualized using an enhanced chemiluminescence system (ECL, GE Healthcare, Piscataway, NJ) and quantified by densitometry analysis using UNSCANIT (Silk Scientific, Inc., Orem, UT). The density ratio of each band compared to its corresponding $\beta$-actin band was determined. The density ratio was normalized to the untreated value which was set to 1 . 


\section{Histology:}

Liver or intestinal sections were fixed in $10 \%$ buffered formalin for 24 hours and embedded in paraffin. Tissue sections were deparaffinized, stained with hematoxylin-eosin (H\&E), and examined by light microscopy $(\times 200$ final magnification).

\section{ALT \& AST assay}

Alanine aminotransferase (ALT) and aspartate aminotransferase (AST) activity were measured as markers of liver injury using commercially available reagents from Thermo Fisher Scientific Inc. (Middletown, VA).

\section{Oil Red $O$ staining}

Frozen liver sections were washed in phosphate buffered saline twice for 5 minutes. Oil-Red-O and $85 \%$ propylene glycol were added with agitation for 15 minutes, followed by washing in tap water.

\section{Detection of apoptosis}

TUNEL staining for apoptosis was performed using ApopTag® Peroxidase In Situ Apoptosis Detection Kit (EMD Millipore Corporation, Billerica, MA).

\section{Detection of acrolein adducts}

Acrolein adducts were detected using an antibody specific for FDP-Lysine acrolein adducts obtained from Cell Sciences. (Canton, MA). Quantitation was done and calculated by ImageJ and MetaMorph microscope. Ten pictures from each liver were used for analysis. 


\section{Immunofluorescence microscopy}

lleum sections were fixed in $10 \%$ buffered formalin for 24 hours and embedded in paraffin. Tissue sections were deparaffinized and incubated with polyclonal rabbit anti-claudin-1, occludin, or ZO-1 antibodies from Invitrogen (Carlsbad, CA) overnight at $4^{\circ} \mathrm{C}$, followed by incubation of fluorescein isothiocyanate-conjugated antibody from Invitrogen (Carlsbad, CA) for 30 minutes at room temperature. Images were examined using a Nikon A1R-A1confocal microscope equipped with a digital image analysis system (Pixera, San Diego,CA)

\section{Statistical analysis}

Statistical analysis was performed using GraphPad Prism version 5.01 for Windows (GraphPad Software, Inc., La Jolla, CA). One-way analysis of variance (ANOVA) followed by Bonferroni post test analysis (for greater than two groups) was used to evaluate significant differences between the compared groups. A pvalue of $<0.05$ was considered statistically significant. Data were expressed as mean \pm SEM. 


\section{CHAPTER 3}

\section{RESULTS SECTION I: LIVER STUDIES.}

In this study, we examined the contributory role of the lipid-derived aldehyde, acrolein, to alcohol-induced liver injury using cultured rodent hepatoma cells (H4IIEC), and the chronic+binge murine model of ALD, also called the NIAAA (National Institute on Alcohol Abuse and Alcoholism) model. This is a wellaccepted mouse model for hepatic steatosis and hepatocellular injury in ALD, and reflects a common drinking pattern in humans, particularly in patients with ALD who are often chronic and binge drinkers $[67,68]$.

\section{Alcohol consumption in mice leads to hepatic acrolein generation and accumulation of acrolein adducts}

In order to investigate the theory that acrolein is a pathogenic mediator of ALD, we first examined whether alcohol consumption led to acrolein buildup in the liver. Although free acrolein is extremely labile and difficult to quantify, it readily reacts with cellular proteins to form covalent adducts which can then be assessed. A marked increase was observed in the levels of acrolein-protein adducts (brown staining of acrolein-FDP-lysine adducts) in the livers of alcohol-fed mice (Figure 7A) compared to controls, showing that alcohol consumption led to the hepatic generation/accumulation of acrolein. The alcohol-induced acrolein adduct accumulation was largely not zone-specific, but was slightly increased close to the portal vein in some animals. Also, both cytoplasmic and nuclear accumulation was 
seen, suggesting that proteins in many subcellular locations were adducted by acrolein. Quantification of acrolein adducts revealed a 3-fold, statistically significant difference between control and alcohol-fed livers (Figure 7A).

To determine the mechanisms underlying alcohol-induced acrolein accumulation, we examined enzymes that primarily metabolize alcohol, namely, $\mathrm{ADH}$ and CYP2E1. Alcohol is known to upregulate CYP2E1 and increase its own metabolism, thereby leading to oxidative stress and enhanced LPO. Hence, it is likely to generate higher levels of the LPO-derived aldehydes, such as acrolein. Accordingly, we examined CYP2E1 and ADH expression in the livers of control versus alcohol-fed mice. As anticipated, alcohol feeding led to a robust increase in CYP2E1 protein levels in the alcohol-fed mice (Figure 7B). However, CYP2E1 mRNA was decreased by alcohol feeding (Figure 7C). Similar effects were seen in hepatic ADH, wherein ADH mRNA was decreased (Figure $7 C$ ) and ADH protein levels were slightly increased by alcohol (Figure 7B). Alcohol-induced increase in CYP2E1 protein in the absence of transcriptional upregulation of the mRNA, has been proposed to be due to stabilization of the protein [69]; whether the same occurs with $A D H$ remains to be investigated. The concentration of acrolein in the liver is modulated not only by its generation but also by the rate of detoxification or removal. Since acrolein is primarily metabolized and cleared via conjugation with glutathione catalyzed by the enzyme glutathione-s-transferase-Pi (GSTP) [18], we also examined the effects of alcohol on hepatic GSTP levels. Alcohol consumption resulted in a decrease in the hepatic levels of both GSTP mRNA (Figure 7C) and GSTP protein (Figure 7B) as seen in alcohol-fed mice compared to control, 
suggesting that the decrease in GSTP may contribute to acrolein accumulation in the livers of alcohol-fed mice. 


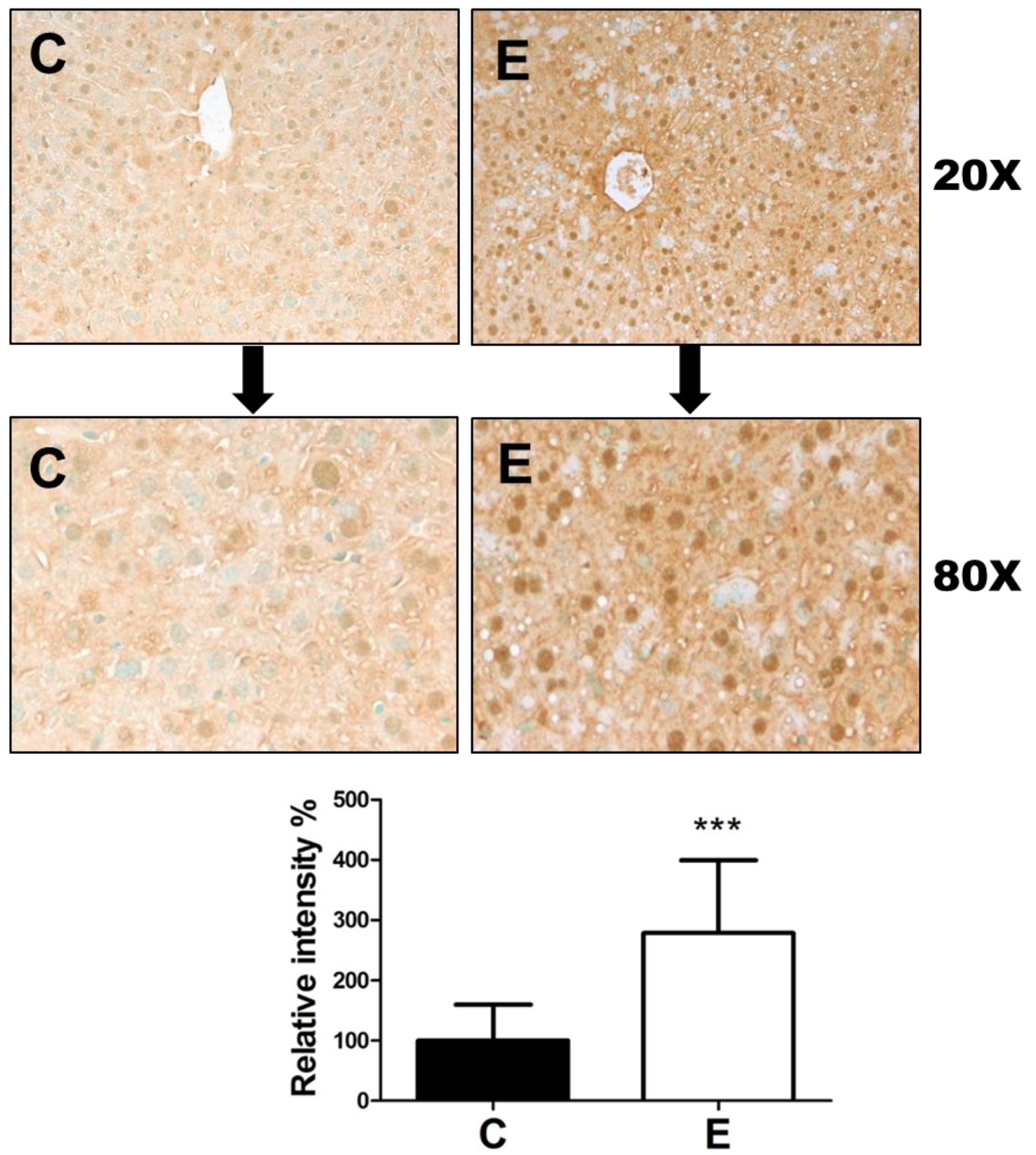

Figure 7A. Alcohol consumption leads to accumulation of acrolein-protein adducts in mice livers.

Top: Accumulation of acrolein adducts in mice livers by immunohistochemistry using specific FDP-lysine antibodies (20x and 80x magnification).

Bottom: Quantification of acrolein adducts in (A). Mean \pm SEM, $n=6$ mice, ${ }^{* * *} p<0.001$ compared to control by student's t-test. $\mathrm{C}=$ Control; $\mathrm{E}=\mathrm{Alcohol}$. 


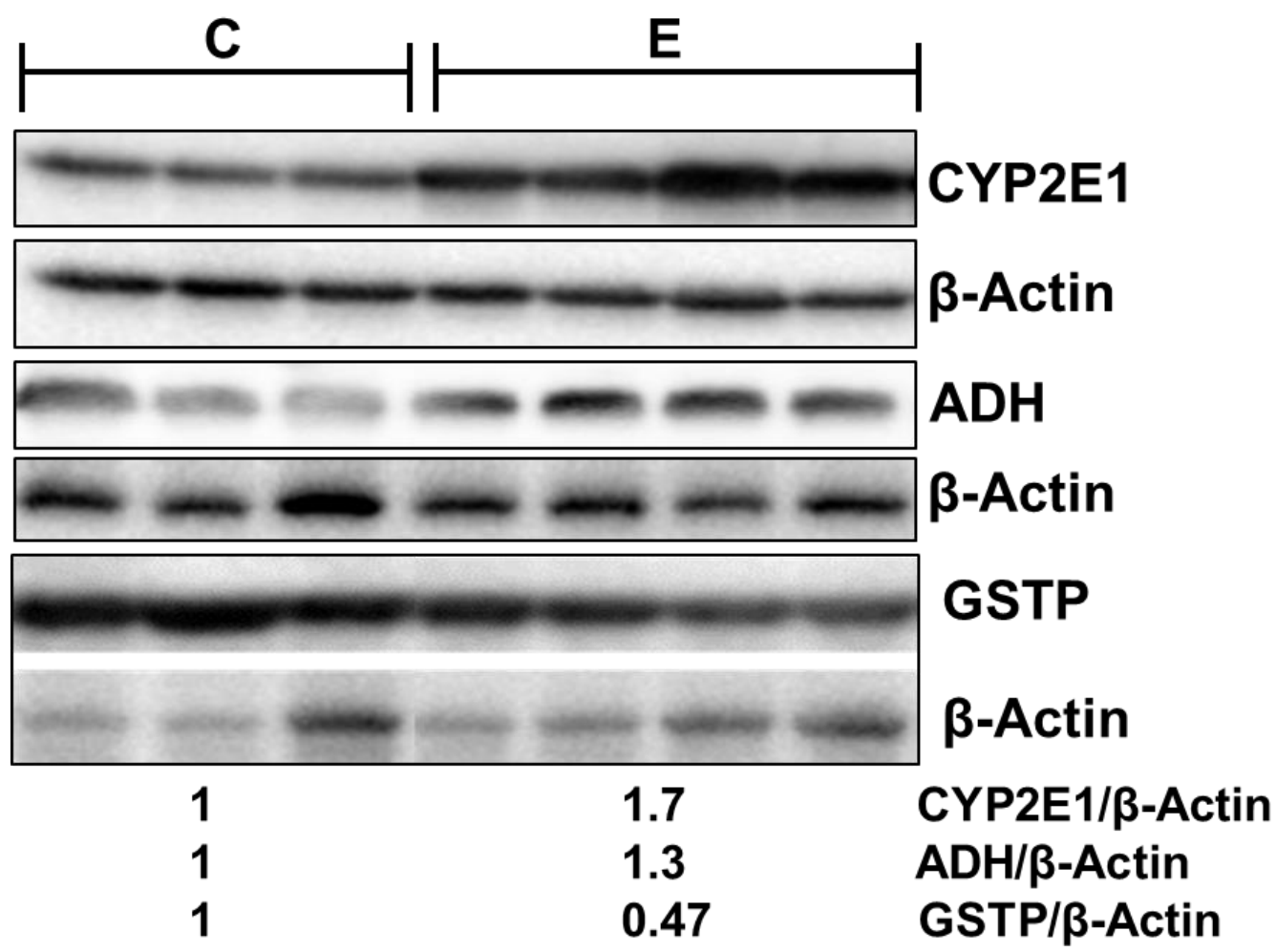

Figure 7B. Alcohol consumption leads to upregulation of ADH and CYP2E1, and downregulation of GSTP in mice livers.

Western blot analysis of ADH, CYP2E1 and GSTP was performed using total cell lysates. Blots were reprobed with antibody to $\beta$-actin to ensure equivalent loading. Densitometry analysis was performed using Imagelab software. Density ratio was calculated using $\beta$-actin as control. Numbers represent the mean of density ratio for each group of mice pair-fed the control or ethanol diet. $\mathrm{C}=$ Control; $\mathrm{E}=$ Alcohol. 


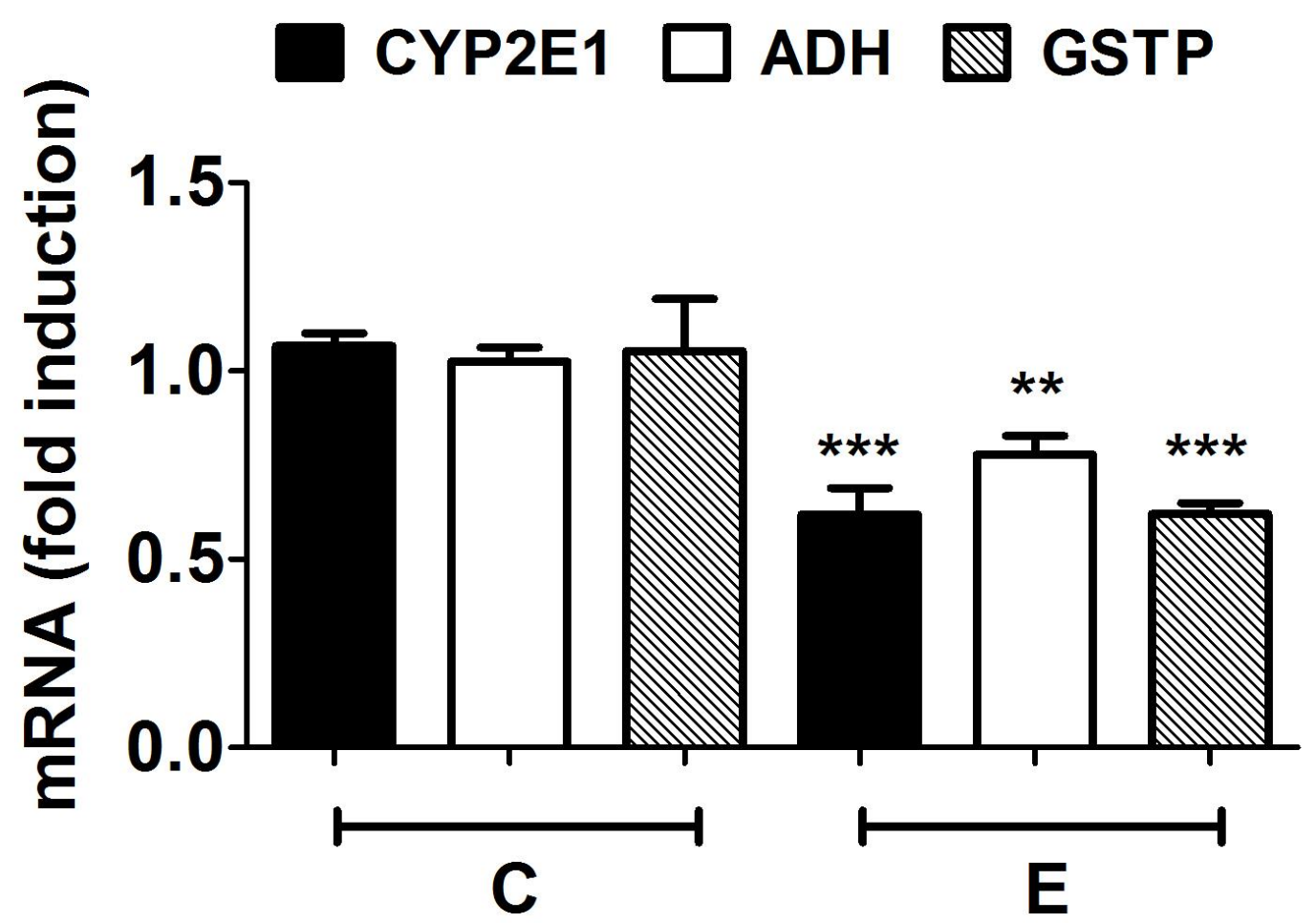

Figure 7C. Alcohol consumption leads to downregulation of ADH, CYP2E1, and GSTP in mice livers.

Total RNA was evaluated for mRNA expression by real-time PCR using primers specific for ADH, CYP2E1 and GSTP genes. Data are presented as the mean \pm SEM $(n=6)$. Statistical analysis was performed using GraphPad Prism Software using one-way ANOVA followed by Bonferroni posttest. ${ }^{* *} \mathrm{p}<0.01,{ }^{* *} \mathrm{p}<0.001$. $\mathrm{C}=$ Control; $\mathrm{E}=$ Alcohol. 
Using cultured rodent hepatic cells that are known to metabolize alcohol (H4IIEC cells), we assessed whether in vitro exposure to alcohol resulted in the accumulation of acrolein adducts, similar to that seen in livers of alcohol-fed mice. Considerable acrolein adduct accumulation was seen in H4IIEC cells that were treated for $24 \mathrm{~h}$ with alcohol $(200 \mathrm{mM})$ compared to untreated cells (Figure $8 \mathrm{~A})$. Alcohol is metabolized in the liver by ADH or CYP2E1 into its primary metabolite, acetaldehyde, which causes increased ROS production leading to enhanced LPO. Based on the acrolein buildup seen in alcohol-exposed mice and cultured cells, we explored the direct role of the alcohol metabolite, acetaldehyde, in acrolein generation and adduct accumulation in hepatocytes. Cells were exposed in vitro to acetaldehyde $(50 \mu \mathrm{M}$ or $100 \mu \mathrm{M})$ for $24 \mathrm{~h}$, and acrolein adduct levels were examined. Similar to alcohol, direct exposure of cells to acetaldehyde resulted in significant acrolein adduct accumulation (Figure 8A). Quantification showed that treatment with alcohol and $100 \mu \mathrm{M}$ acetaldehyde caused a statistically significant increase in acrolein adducts, and a similar trend (but not statistically significant) was seen with the lower concentration of acetaldehyde (Figure 8A). Once formed, acetaldehyde is further metabolized by aldehyde dehydrogenase (ALDH) enzymes, particularly ALDH2, which is also known to metabolize and remove reactive unsaturated aldehydes such as acrolein. Hence, we tested whether ALDA1, a known agonist of ALDH2, affected alcohol-induced acrolein buildup. Pretreatment of cells with ALDA1 prior to alcohol exposure considerably decreased the accumulation of acrolein adducts (Figure 8B), showing that stimulating ALDH2 attenuated alcohol-induced acrolein accumulation. Further, to determine whether 
acrolein buildup was dependent on alcohol metabolism, H4IIEC cells were also exposed to various known inhibitors of alcohol metabolism, prior to alcohol exposure. Treatment of cells with either pyrazole or 4-methylpyrazole (which suppress both ADH and CYP2E1 dependent alcohol metabolism [70]) substantially attenuated the accumulation of alcohol-induced acrolein adducts (Figure 8B). In comparison, allyl sulfide, which is a more selective CYP2E1 inhibitor, was less effective in blocking alcohol-induced acrolein buildup, suggesting that both ADH and CYP2E1 pathways led to acrolein generation, and that inhibition of only one metabolic pathway was insufficient to eliminate total acrolein adduct accumulation (Figure 8B). Thus, alcohol consumption and metabolism causes acrolein accumulation by simultaneously upregulating enzymes that lead to acrolein generation and downregulating the enzyme that metabolizes and removes acrolein. In order to further investigate the role of downregulation of GSTP in the alcohol-induced buildup of acrolein adducts, we used siRNA in H4IIEC cells to effectively inhibit GSTP mRNA and protein (Figure 8C). Genetic inhibition of GSTP by siRNA led to an increase in the accumulation of acrolein adducts (Figure 8D) following treatment with alcohol or acrolein, which was not seen in cells transfected with scrambled RNA control. These data confirmed that the downregulation of GSTP by alcohol may be a novel mechanism that contributes to enhanced alcohol-induced hepatic acrolein generation and adduct accumulation. Thus, our data show that alcohol consumption and metabolism causes acrolein accumulation by simultaneously upregulating 
enzymes that lead to acrolein generation and downregulating the enzyme that metabolizes and removes acrolein. 

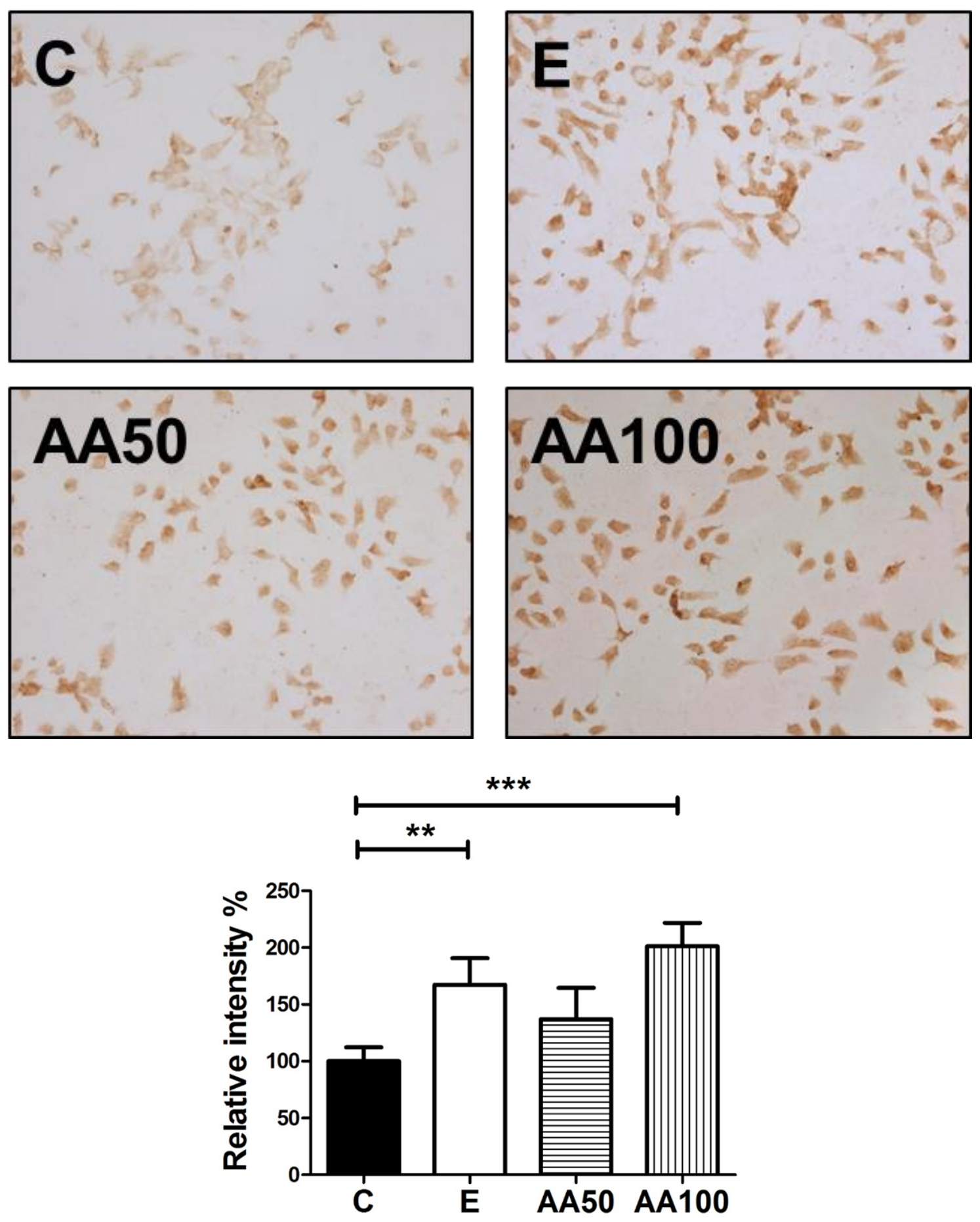

Figure 8A. Effect of acetaldehyde on alcohol-induced acrolein adduct accumulation in cultured hepatic cells.

Top: Accumulation of acrolein adducts by immunocytochemistry using specific FDP-lysine antibodies (20x magnification) in H4IIEC cells treated for $24 \mathrm{~h}$ as 
follows: Untreated control cells $(C)$ or cells treated with $200 \mathrm{mM}$ alcohol $(\mathrm{E}), 50 \mu \mathrm{M}$ acetaldehyde (AA50) or 100 $\mu \mathrm{M}$ acetaldehyde (AA100).

Bottom: Quantification of acrolein adducts in (A). Mean \pm SEM, $n=4,{ }^{* *} p<0.01$ and ${ }^{* * *} p<0.001$ compared to control by ANOVA - Bonferroni analysis. 

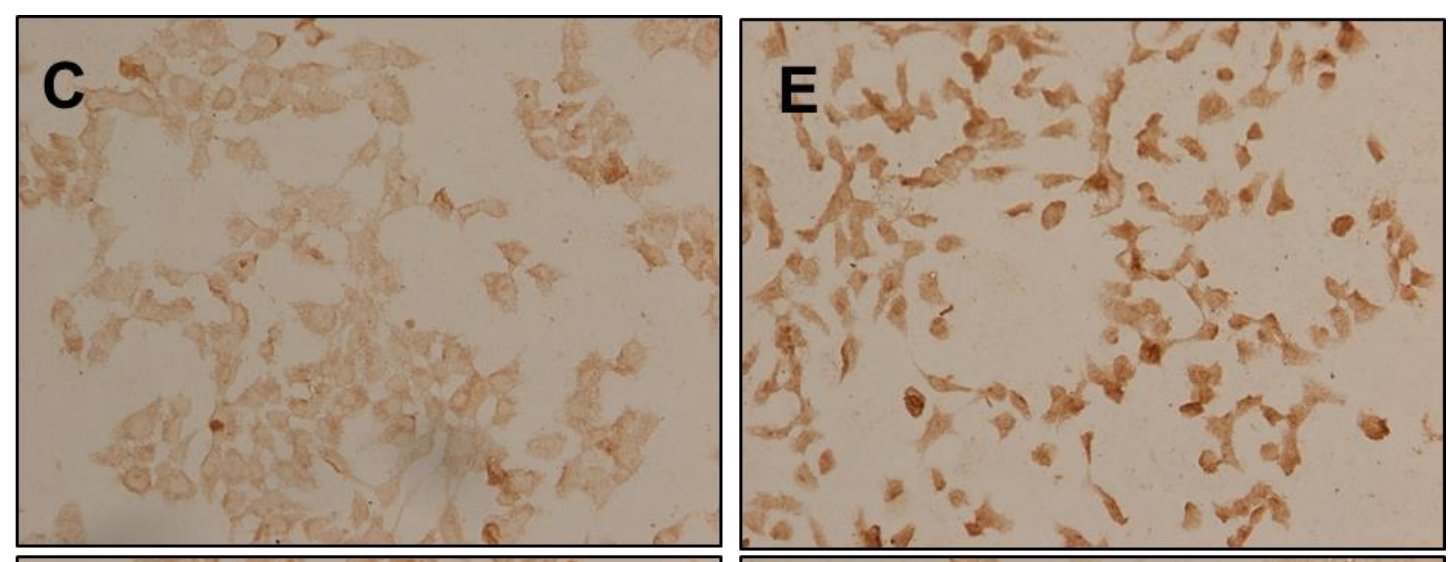

\section{ALDA1+E}
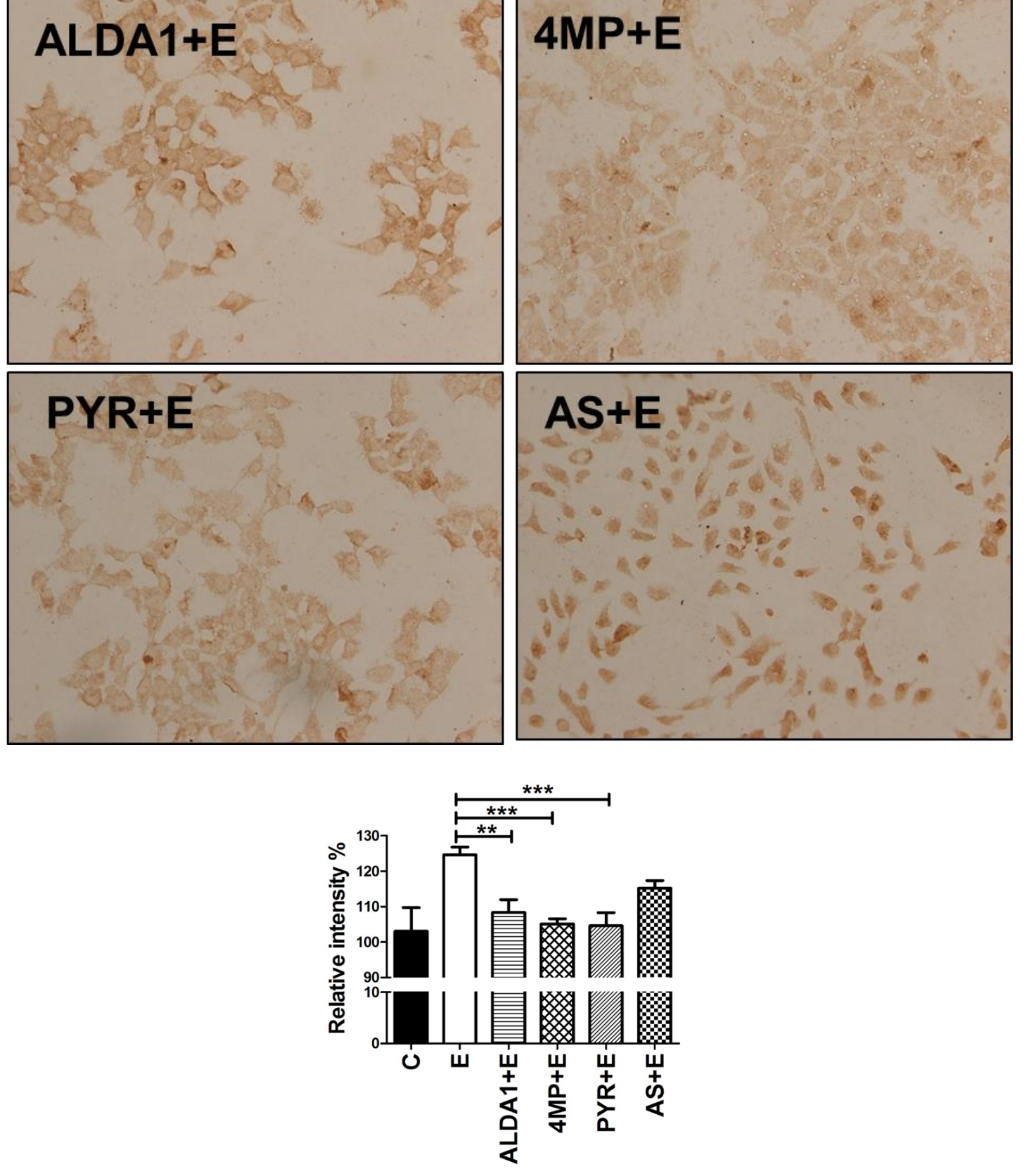
Figure 8B. Effect of pharmacologic modulators of alcohol metabolism on alcohol-induced acrolein adduct accumulation in cultured hepatic cells. Top: Accumulation of acrolein adducts (20x magnification) in H4IIEC cells treated for $24 \mathrm{~h}$ as follows: Untreated control cells (C); cells treated with $200 \mathrm{mM}$ alcohol alone (E); or 200mM alcohol in the presence of $10 \mu \mathrm{M}$ Alda1 (ALDA1+E); $10 \mu \mathrm{M}$ 4-methyl pyrazole (4MP+E); $10 \mu \mathrm{M}$ Pyrazole (PYR+E); or $10 \mu \mathrm{M}$ allyl sulfide $(A S+E)$.

Bottom: Quantification of acrolein adducts in (C). Mean \pm SEM, $n=4,{ }^{* *} p<0.01$ and ${ }^{* * *} \mathrm{p}<0.001$ compared to control by ANOVA - Bonferroni analysis. 

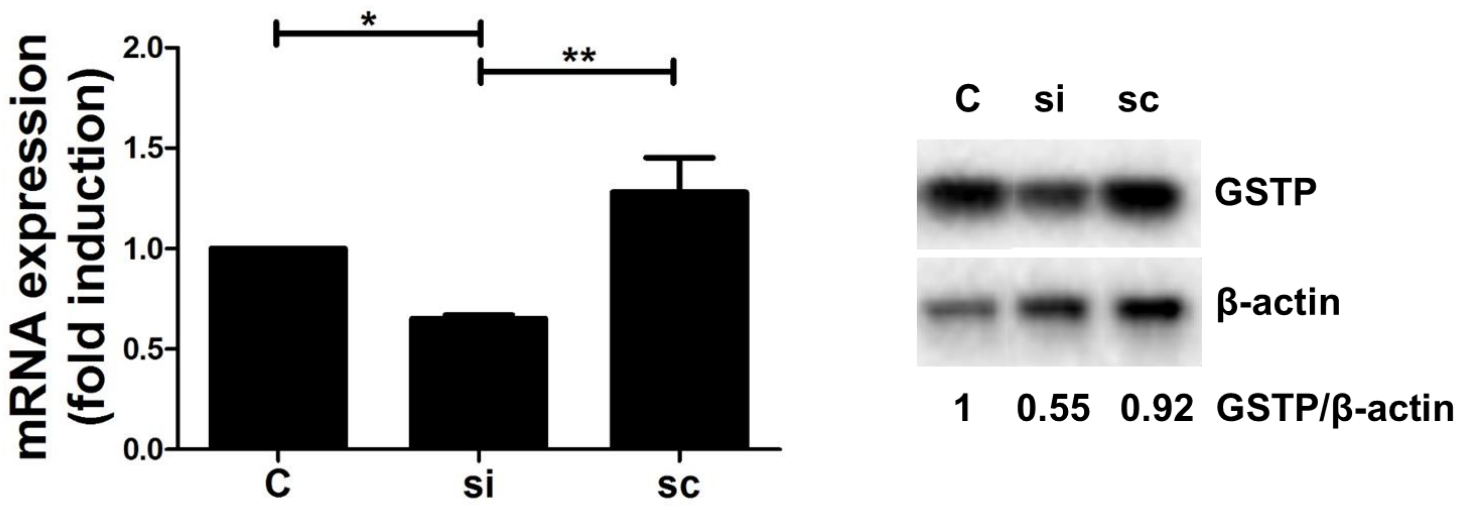

Figure 8C. Effect of inhibition of GSTP on alcohol-induced acrolein adduct accumulation in cultured hepatic cells.

Inhibition of GSTP mRNA and protein by siRNA transfection in H4IIEC cells. For PCR analysis ${ }^{*} p<0.05$ compared to control. For Western blot, the numbers represent mean densitometry ratios normalized to $\beta$-actin. $\mathrm{C}=$ =Control; si=GSTP siRNA; and sc=scrambled RNA as negative control. 

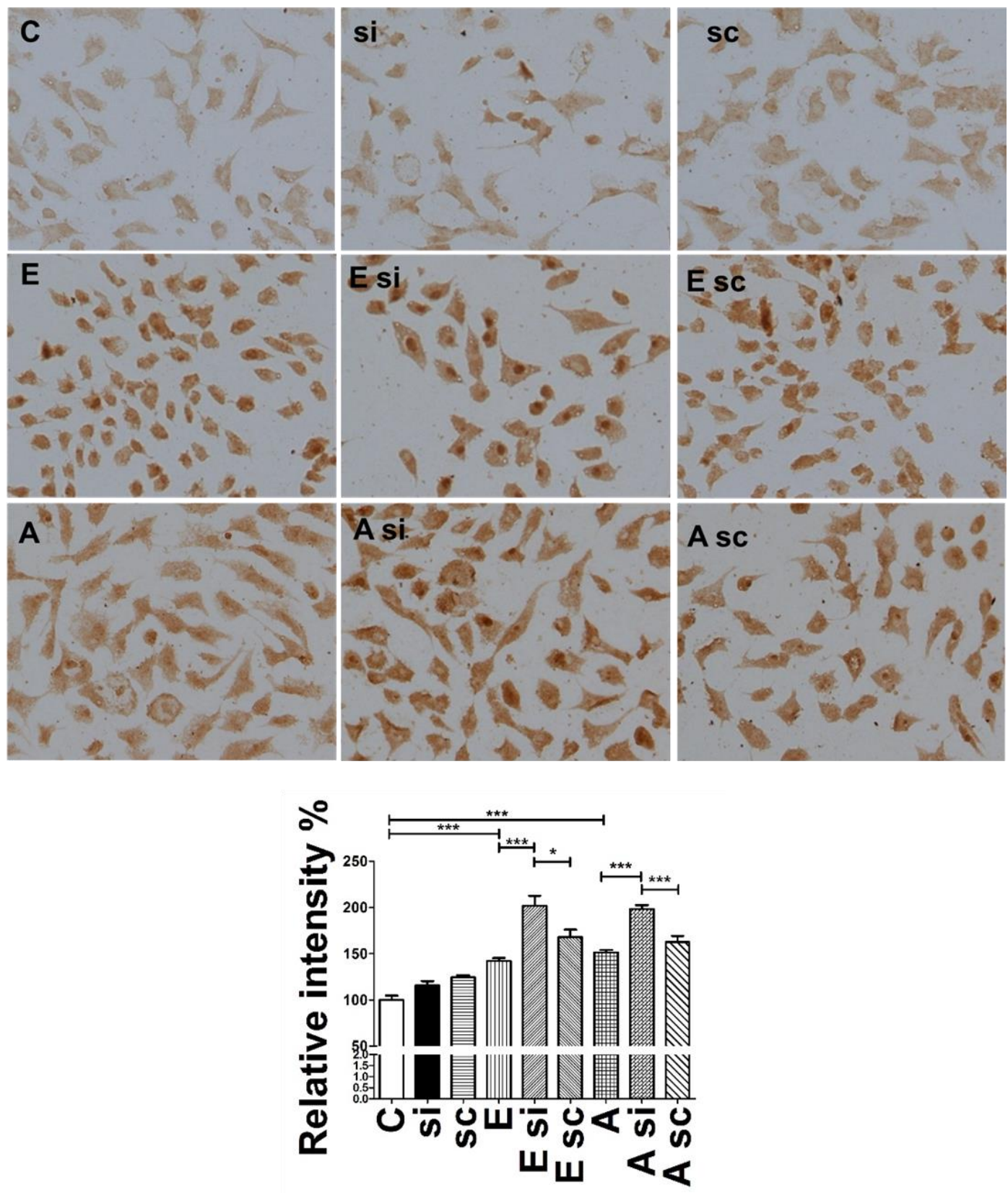

Figure 8D. Effect of inhibition of GSTP on alcohol-induced acrolein adduct accumulation in cultured hepatic cells.

Accumulation of acrolein adducts (20x magnification) in transfected H4IIEC cells, and quantification of acrolein adducts. ${ }^{*} p<0.05$ and ${ }^{* * *} p<0.001$ compared to control by ANOVA - Bonferroni analysis. $C=$ Control; $E=200 \mathrm{mM}$ Alcohol; $A=20 \mu \mathrm{M}$ Acrolein; si=GSTP siRNA; and sc=scrambled RNA as negative control. 


\section{Alcohol-induced hepatic acrolein adduct accumulation is associated with ER stress, with minimal activation of ER adaptive/protective responses}

The alcohol-induced accumulation of acrolein-adducted proteins in the liver is likely to increase the burden on the cellular ER protein folding machinery, which if overwhelmed, can trigger ER stress. Indeed, in our study, alcohol feeding and the resultant acrolein adduct accumulation was associated with upregulation of the prototypical ER stress markers, activating transcription factors ATF3 and ATF4, at the mRNA (Figure 9A) and protein levels (Figure 9B). The increase in ATF3 and ATF4 was attributed to phospho-activation of the upstream signaling proteins, PERK and eukaryotic translation initiation factor 2A, (elF2 $\alpha$ ) (Figure 9C). 


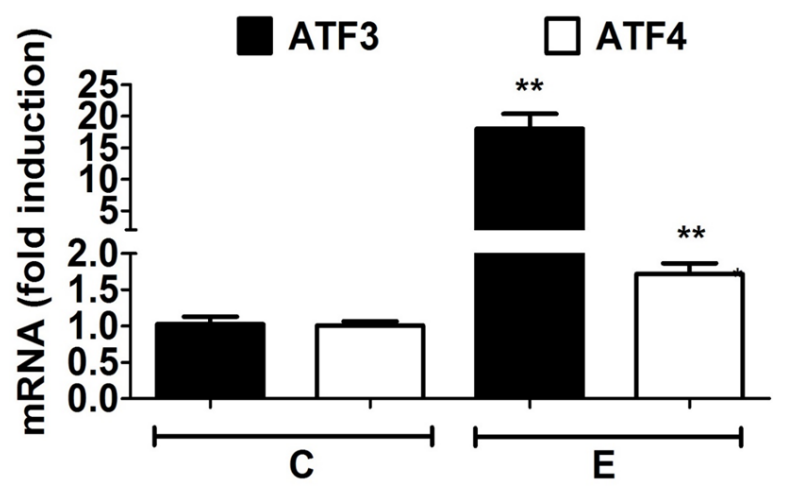

Figure 9A. Alcohol-induced accumulation of acrolein adducts causes hepatic ER stress in mice livers.

Total RNA was evaluated for mRNA expression by real-time PCR using primers specific for ATF3 and ATF4 genes. Data are presented as the mean \pm SEM $(n=6)$. Statistical analysis was performed using Student's t-test. ${ }^{* *} \mathrm{p}<0.01$. $\mathrm{C}=$ Control; $\mathrm{E}=$ Alcohol. 

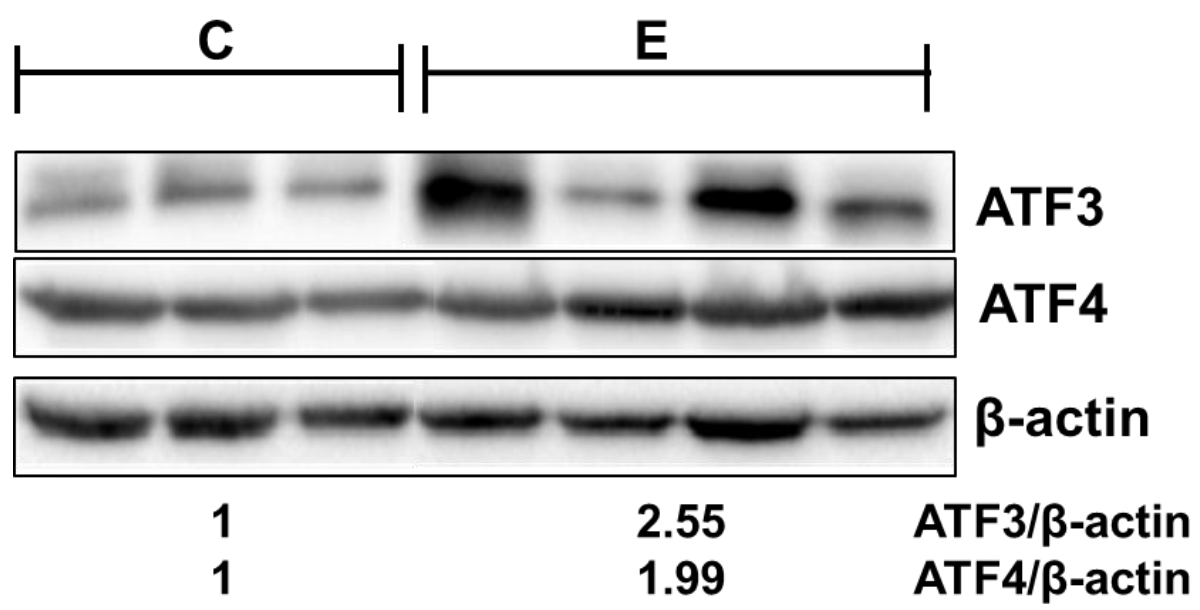

Figure 9B. Alcohol-induced accumulation of acrolein adducts causes hepatic ER stress in mice livers.

Western blot analysis of ATF3 and ATF4 was performed using total cell lysates.

Blots were reprobed with antibody to $\beta$-actin to ensure equivalent loading.

Densitometry analysis was performed using Imagelab software. Density ratio was calculated using $\beta$-actin as control. Numbers represent the mean of density ratio for each group of mice pair-fed control or ethanol diet. $\mathrm{C}=$ Control; $\mathrm{E}=\mathrm{Alcohol}$. 

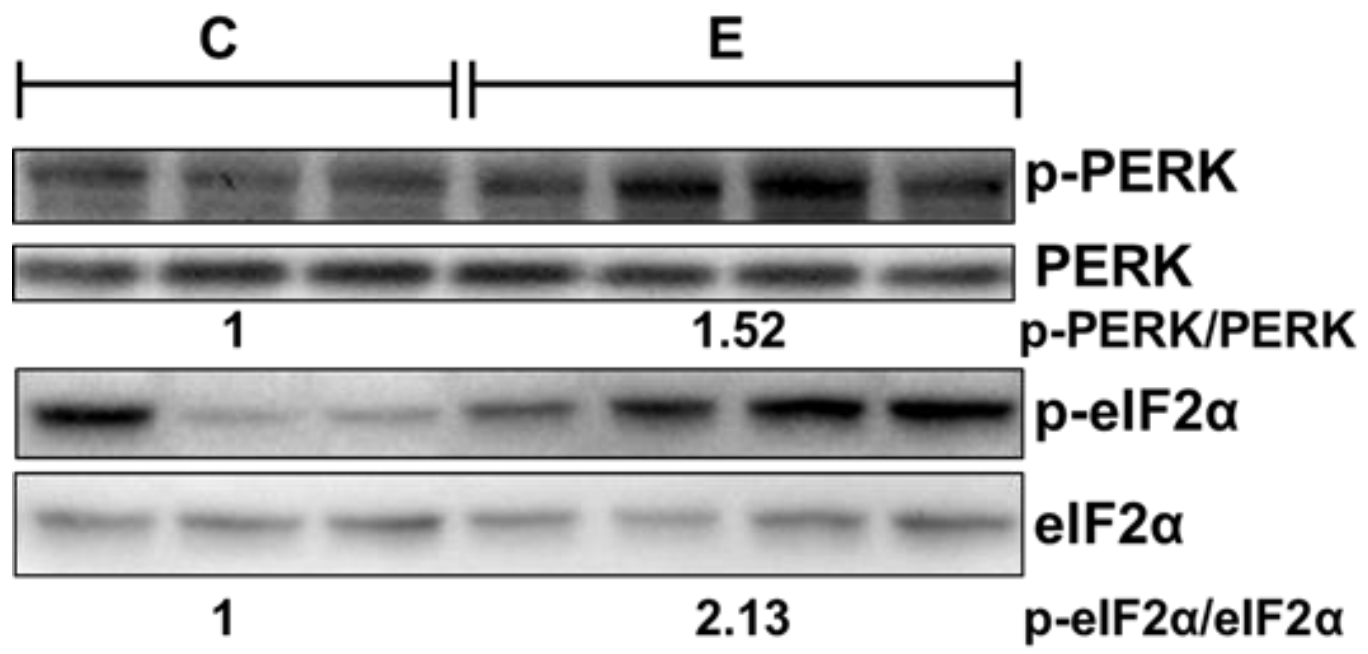

Figure 9C. Phospho-PERK and phospho-elF2 $\alpha$ protein levels. Western blot analysis of Phospho-PERK and phospho-elF2 $\alpha$ was performed using total cell lysates. Blots were reprobed with antibody to $\beta$-actin to ensure equivalent loading. Densitometry analysis was performed using Imagelab software. Density ratio was calculated using $\beta$-actin, total PERK or elF2 $\alpha$ as control. Numbers represent the mean density ratio for each group of mice pair-fed control or ethanol diet. $\mathrm{C}=$ Control; $\mathrm{E}=\mathrm{Al}$ cohol. 
Further, the effect of alcohol on the adaptive/protective responses associated with ER stress was also studied by examining the gene expression of hallmark ER chaperones, GRP78 and GRP94; these chaperones can promote protein folding, and thereby relieve ER stress and provide protection. A small (but statistically significant) increase was seen in the mRNA levels of both GRP78 and GRP94 (Figure 10A); while at the protein level, only GRP78 was marginally elevated (Figure 10B) in alcohol fed mice, suggesting that cellular ER-adaptive responses were not robustly activated. Next, to determine the underlying cause, we examined the transcription factors that are thought to regulate chaperone gene expression, namely, ATF6 and X-box binding protein-1 (XBP1). Activation of ATF6 by proteolytic cleavage from the inactive $\sim 90 \mathrm{kDa}$ fragment into the active $\sim 50 \mathrm{kDa}$ cleaved form (Figure 10C) was seen in alcohol-fed mice, showing that the ATF6mediated ER stress pathway was also triggered in this model of alcohol consumption in mice. XBP1 is activated by unconventional splicing of XBP1 mRNA due to ER stress-induced activation of IRE1 endonuclease activity via oligomerization, and subsequent autophosphorylation. Accordingly, we examined IRE1 phosphorylation and XBP1 splicing in alcohol-fed mice and observed that alcohol consumption slightly decreased IRE1 phosphorylation (Figure 10C). Also, we observed a lack of spliced XBP1 (Figure 10D), and consequently, no induction of XBP1s protein in the majority of livers of alcohol-fed mice (Figure 10E). Interestingly, the unspliced XBP1u ( 26kDa) was slightly increased in alcohol fed mice at the mRNA and protein level; the relevance of this finding remains to be clarified. These data show that alcohol feeding induced ER stress and activated 
ATF6, but failed to splice XBP1, and these actions did not upregulate ER chaperones. 


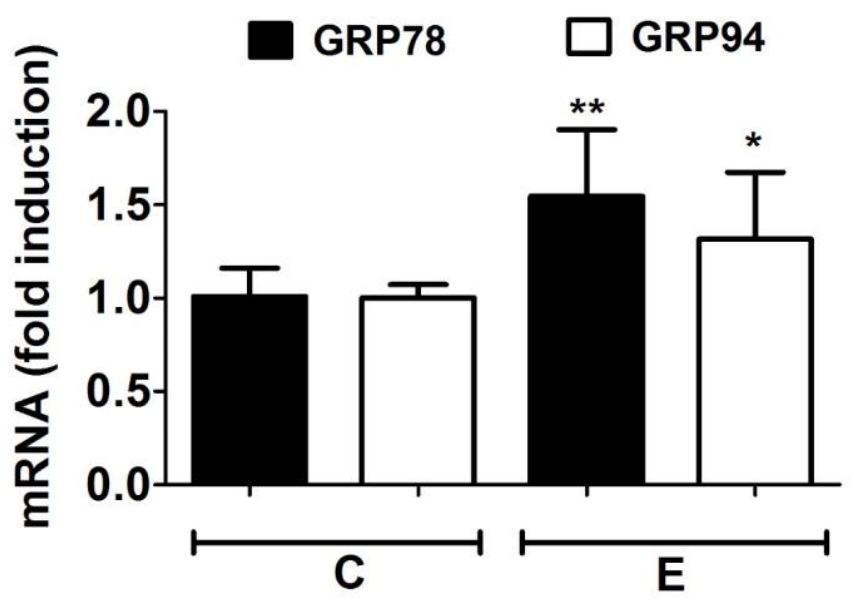

Figure 10A. Alcohol-induced accumulation of acrolein adducts induces ER chaperone mRNA expression in mice livers.

Total RNA was evaluated for chaperone gene expression by real-time PCR using primers specific for GRP78 and GRP94 genes. Data are presented as the mean \pm SEM $(n=6)$. Statistical analysis was performed using Student's t-test. " $p<0.05$ and ${ }^{* *} \mathrm{p}<0.01$. $\mathrm{C}=$ Control; $\mathrm{E}=$ Alcohol. 


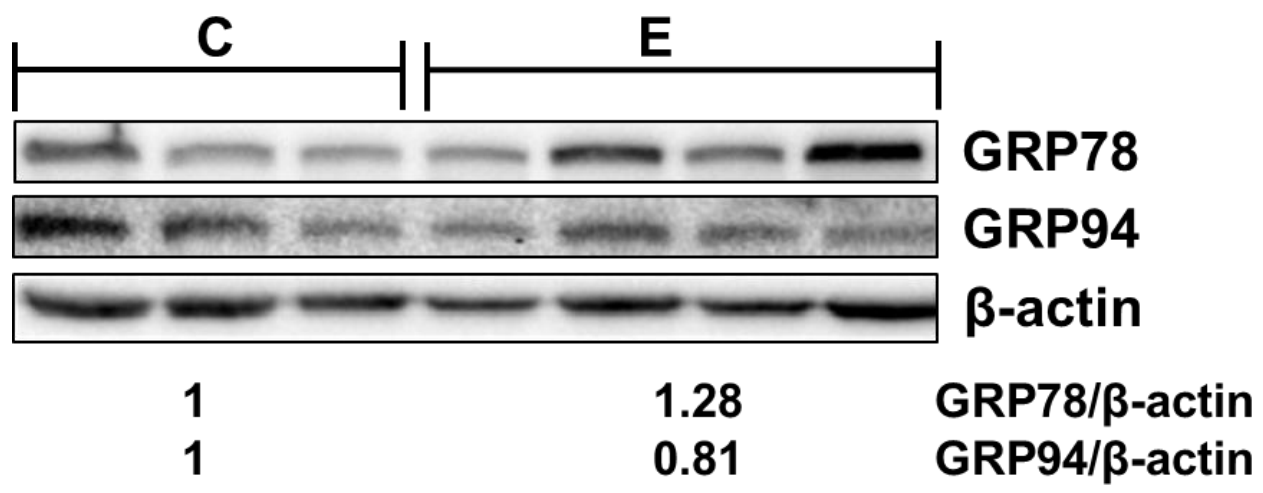

Figure 10B. Alcohol-induced accumulation of acrolein adducts induces minimal ER chaperone proteins in mice livers.

Western blot analysis of GRP78 and GRP94 was performed using total cell lysates. Blots were reprobed with antibody to $\beta$-actin to ensure equivalent loading. Densitometry analysis was performed using Imagelab software. Density ratio was calculated using $\beta$-actin as control. Numbers represent the mean density ratio for each group of mice pair-fed control or ethanol diet. $\mathrm{C}=\mathrm{Control}$; $\mathrm{E}=$ Alcohol. 

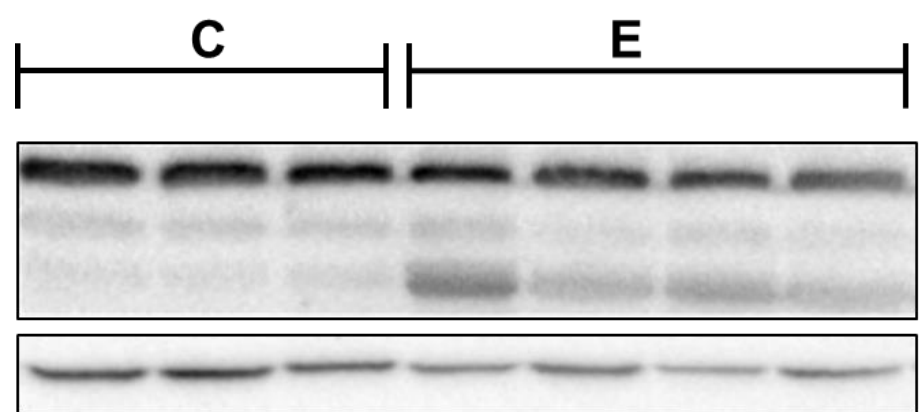

ATF6

Cleaved ATF6

$\beta$-actin

1

0.45

ATF6/ $\beta$-actin

1

16

Cleaved ATF6/ $\beta$-actin

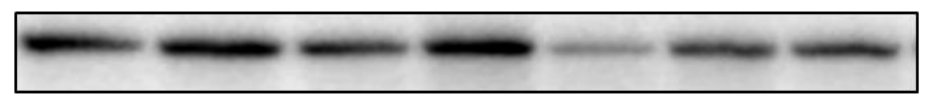

p-IRE

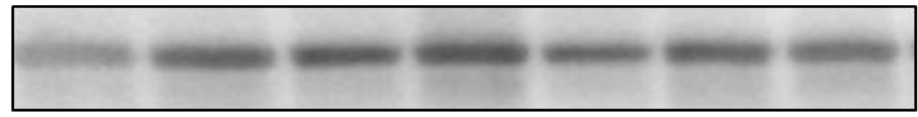

IRE

1

0.72

p-IRE/IRE

Figure 10C. Alcohol-induced accumulation of acrolein adducts activates ATF6 cleavage and decreases IRE phosphorylation in mice livers.

Western blot analysis of ATF6 and cleaved/active ATF6, and phospho-IRE1 and total IRE1 was performed using total cell lysates. Blots were reprobed with antibody to $\beta$-actin to ensure equivalent loading. Densitometry analysis was performed using Imagelab software. Density ratio was calculated using $\beta$-actin or total IRE1 as control. Numbers represent the mean density ratio for each group of mice pair-fed control or ethanol diet. $\mathrm{C}=$ Control; $\mathrm{E}=\mathrm{Alcohol}$. 

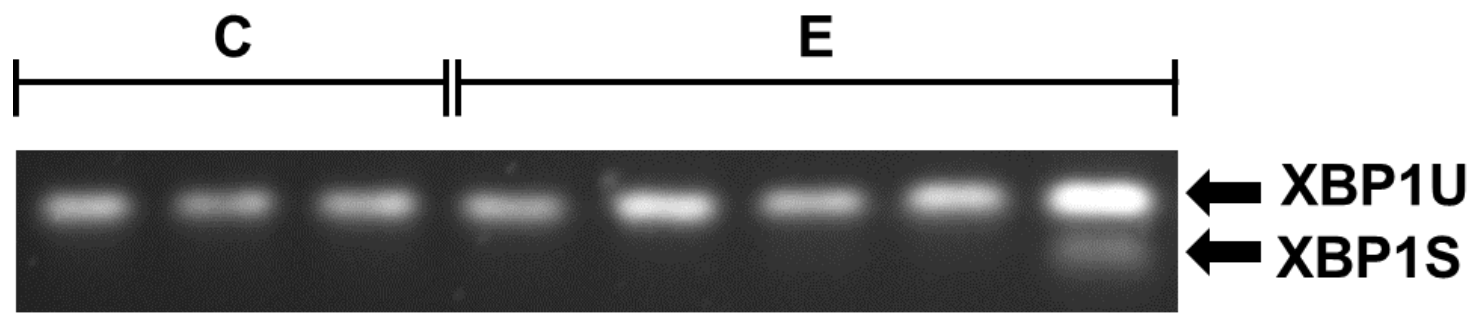

Figure 10D. Alcohol-induced accumulation of acrolein adducts does not induce XBP1 splicing in mice livers.

XBP1 splicing by semi-quantitative endpoint reverse transcription-PCR visualized by agarose gel electrophoresis. 

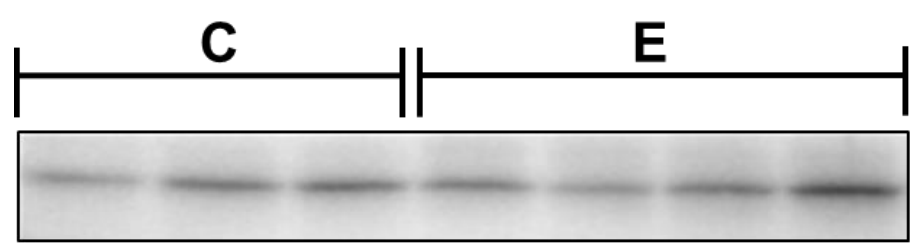

\section{XBP1s}

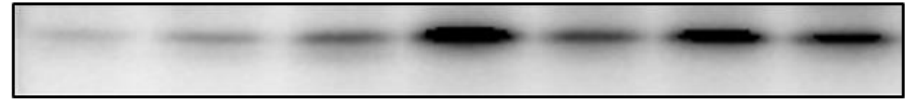

\section{XBP1u}

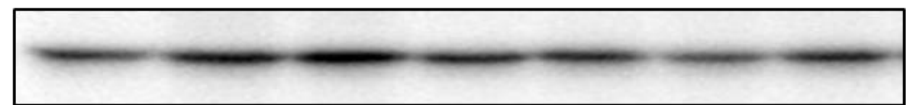

$\beta$-Actin

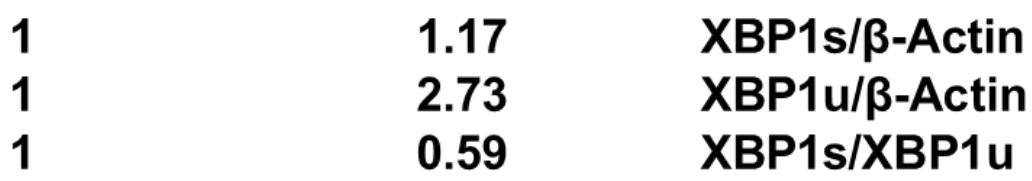

Figure 10E. Alcohol-induced accumulation of acrolein adducts does not induce spliced XBP1 protein in mice livers.

Western blot analysis of XBP1s and XBP1u was performed using total cell lysates. Blots were reprobed with antibody to $\beta$-actin to ensure equivalent loading. Densitometry analysis was performed using Imagelab software. Density ratio was calculated using $\beta$-actin as control. Numbers represent the mean density ratio for each group of mice pair-fed control or ethanol diet. $\mathrm{C}=\mathrm{Control}$; $\mathrm{E}=$ Alcohol. 


\section{Acrolein adduct accumulation and ER stress occur concurrently with proapoptotic signaling}

Uncontrolled ER stress in cells can result in apoptosis, which is thought to occur by various proapoptotic pathways [71]. Accordingly, we examined the effects of alcohol consumption on relevant ER-associated apoptotic signaling in alcohol fed mice. The activation of IRE1 leads to its interaction with TRAF2 and ASK1, and subsequent activation of the mitogen/stress kinase, JNK, which is associated with ER stress-induced apoptosis. The sustained activation of JNK by phosphorylation is implicated in hepatocyte apoptosis and several forms of liver injury [72]. Hepatocytes express both isozymes JNK1 (46kDa) and JNK2 (54kDa) which regulate inflammation, cell proliferation and death in a cell-type dependent and contextual manner. Our data show that alcohol consumption caused significant phospho-activation of both JNK1 and JNK2, while total JNK was very slightly reduced compared to control mice (Figure 11A).

Another relevant ER-driven apoptotic pathway involves the proteolytic activation of the ER-resident caspase-12, which is known to subsequently activate caspase-9 and caspase-3, resulting in apoptotic cell death [73]. Alcohol consumption also caused proteolytic activation of caspase-12, as demonstrated by a decrease in pro-caspase-12 $(\sim 55 \mathrm{kDa})$ and a concurrent increase in the cleaved form ( 38kDa) (Figure 11B). The third, and possibly the most well characterized, pathway of ER stress-induced apoptosis is via upregulation of $\mathrm{CHOP}$, which is primarily responsible for ER stress-induced cell death [74, 75]. Activated $\mathrm{CHOP}$ leads to alterations in $\mathrm{Bcl} 2$ and $\mathrm{BAX}$ leading to activation of the 
mitochondrial death pathway that is known to be involved in alcohol-induced liver injury. The CHOP gene promoter contains binding sites for transcription factors ATF4 and ATF6 which are both activated by alcohol (Figures 9 and 10, respectively). Correspondingly, alcohol consumption resulted in a $\sim 5$-fold upregulation of CHOP mRNA (Figure 11C) and a concomitant increase in $\mathrm{CHOP}$ protein (Figure 11D). Thus, alcohol consumption and consequent acrolein build up in mice activates ER-associated proapoptotic signaling pathways. 

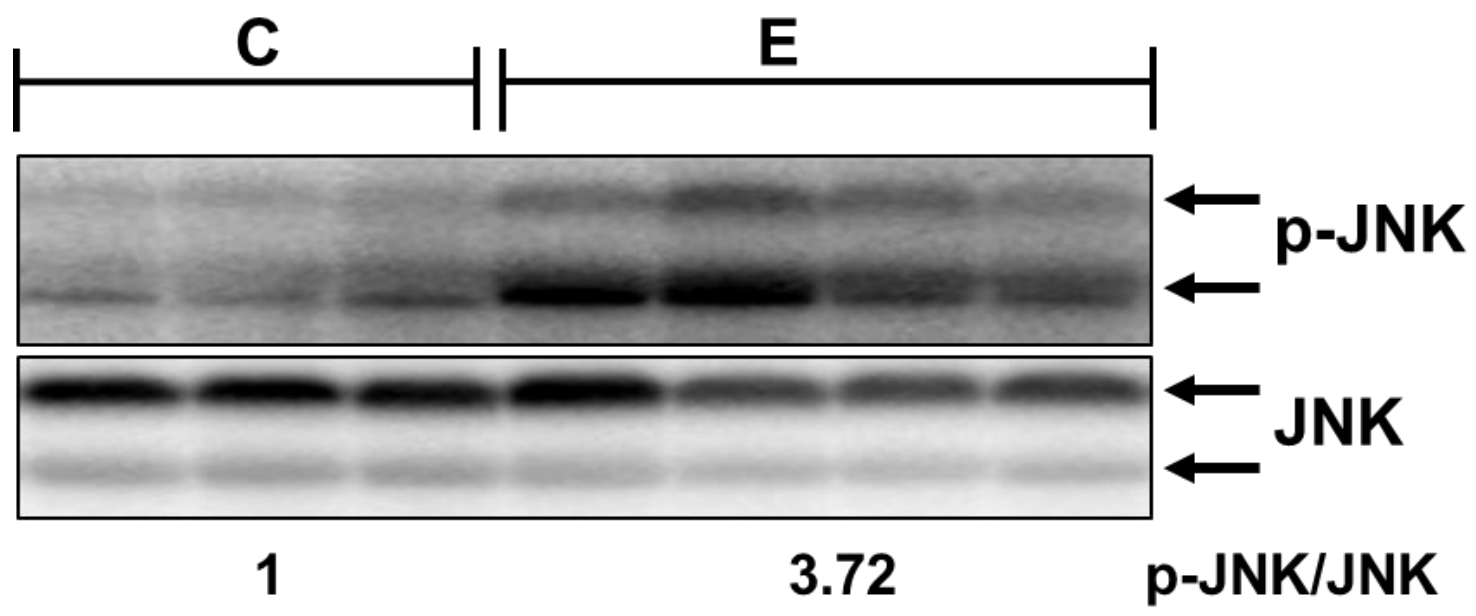

Figure 11A. Alcohol-induced hepatic acrolein build-up and consequent ER stress leads to proapoptotic signaling in mice livers.

Western blot analysis of Phospho-JNK and total JNK was performed using total cell lysates. Blots were reprobed with antibody to $\beta$-actin to ensure equivalent loading. Densitometry analysis was performed using Imagelab software. Density ratio was calculated using total JNK or $\beta$-actin as control. Numbers represent the mean density ratio for each group of mice pair-fed control or ethanol diet. $\mathrm{C}=$ Control; $\mathrm{E}=$ Alcohol. 


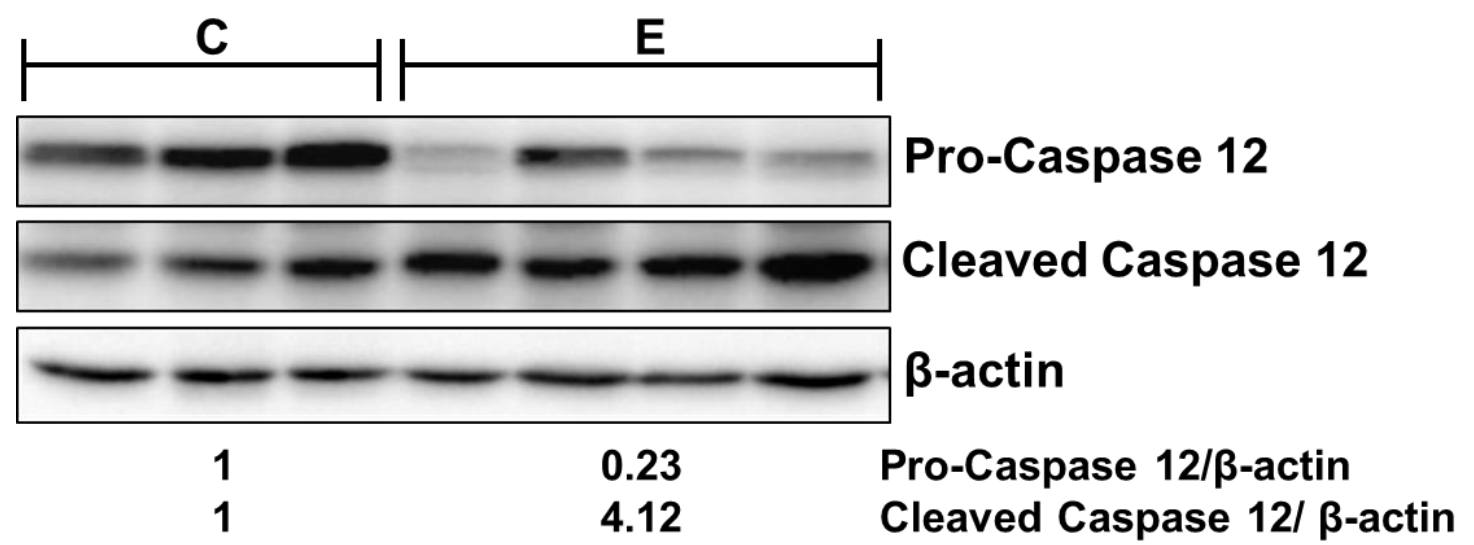

Figure 11B. Alcohol-induced hepatic acrolein build-up and consequent ER stress leads to proapoptotic signaling in mice livers.

Western blot analysis of Pro- and cleaved/active caspase 12 protein levels was performed using total cell lysates. Blots were reprobed with antibody to $\beta$-actin to ensure equivalent loading. Densitometry analysis was performed using Imagelab software. Density ratio was calculated using $\beta$-actin as control. Numbers represent the mean density ratio for each group of mice pair-fed control or ethanol diet. $\mathrm{C}=$ Control; $\mathrm{E}=\mathrm{Alcohol}$. 


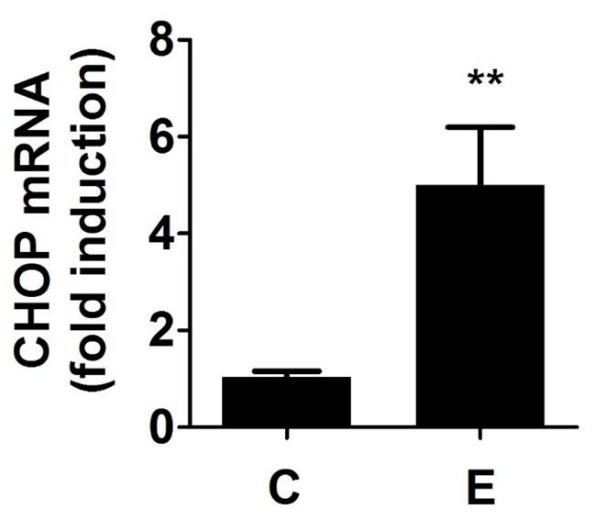

Figure 11C. Alcohol-induced hepatic acrolein build-up and consequent ER stress leads to proapoptotic signaling in mice livers.

Total RNA was evaluated for mRNA expression by real-time PCR using primers specific for CHOP genes. Data are presented as the mean \pm SEM $(n=6)$.

Statistical analysis was performed using Student's t-test. ${ }^{* *} \mathrm{p}<0.01$. C=Control; $E=$ Alcohol. 

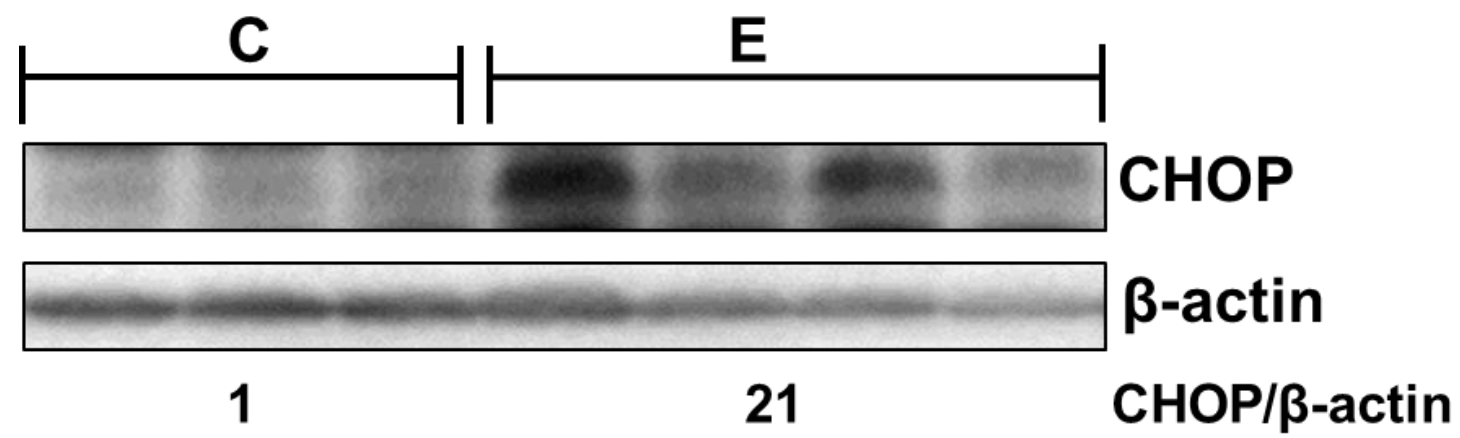

Figure 11D. Alcohol-induced hepatic acrolein build-up and consequent ER stress leads to proapoptotic signaling in mice livers.

Western blot analysis of $\mathrm{CHOP}$ was performed using total cell lysates. Blots were reprobed with antibody to $\beta$-actin to ensure equivalent loading. Densitometry analysis was performed using Imagelab software. Density ratio was calculated using $\beta$-actin as control. Numbers represent the mean density ratio for each group of mice pair-fed control or ethanol diet. $\mathrm{C}=$ Control; $\mathrm{E}=\mathrm{Alcohol}$. 


\section{Hepatic acrolein accumulation and ER stress are accompanied by steatosis, hepatocyte apoptosis and liver injury}

Previous studies have shown that hepatic ER stress is causally linked to steatosis [76]. We examined fat accumulation in the liver. Consistent with hepatic acrolein and ER stress, we observed substantial microvesicular and macrovesicular hepatic steatosis in alcohol-exposed mice compared to controls, as seen by lipid droplet accumulation by Hematoxylin and Eosin (H\&E) and Oil Red O staining (Figure 12A). Further, the activation of proapoptotic signaling culminated in apoptotic cell death in the livers of alcohol-fed mice, as seen by a significant increase in TUNEL (Terminal deoxynucleotidyl transferase dUTP nick-end labeling assay) positive staining in the livers of alcohol-fed mice compared to control (Figure 12A), which led to greater liver injury as shown by elevated serum ALT (Alanine aminotransferase) and AST (Aspartate aminotransferase) (Figure 12B). Thus, concomitant with alcohol-induced accumulation of acrolein and ER stress, an increase was observed in hepatic steatosis and hepatocyte apoptosis, leading to liver injury in alcohol-fed mice. 


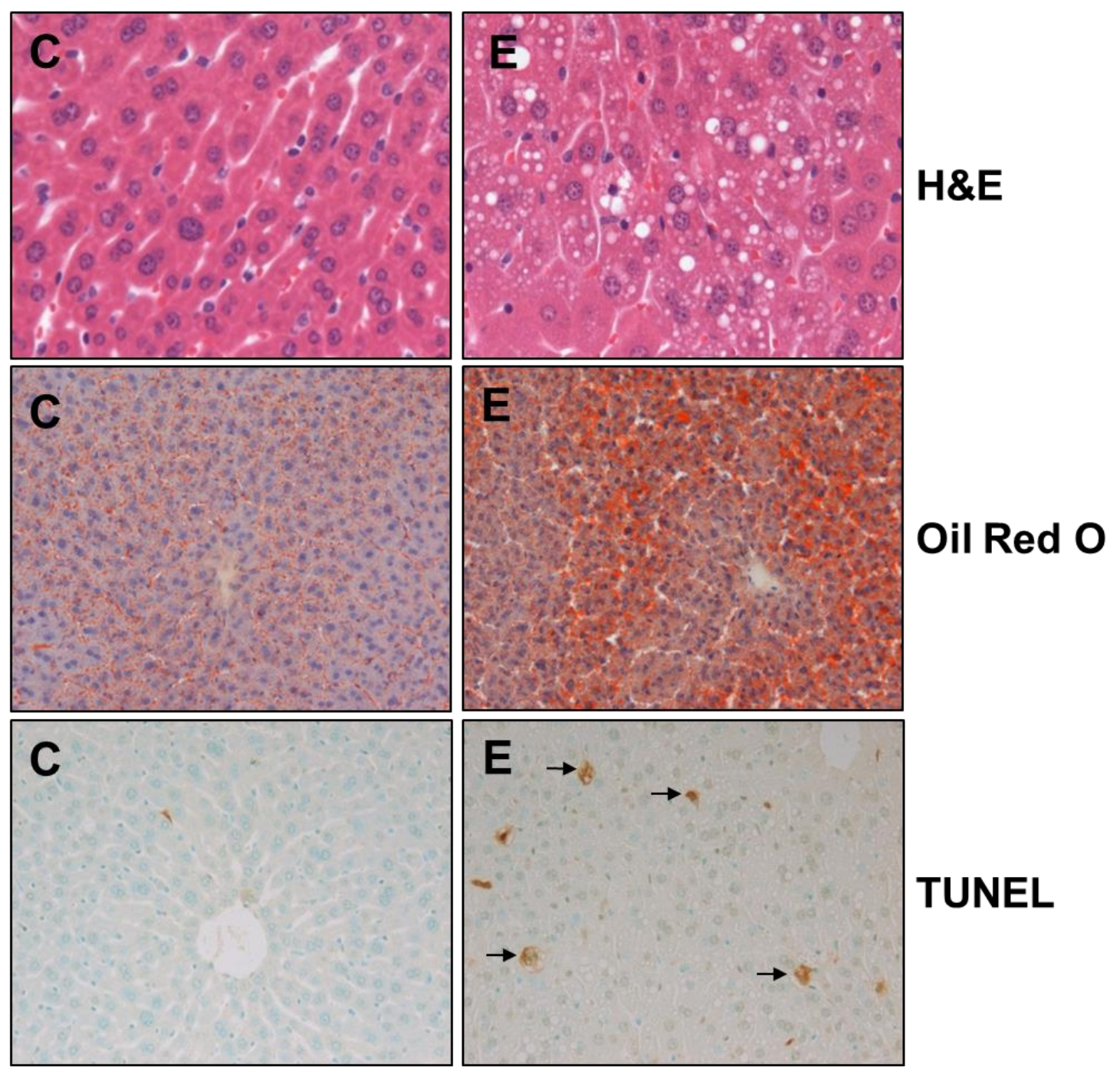

Figure 12A. Alcohol-induced acrolein and ER stress leads to steatosis, hepatocyte apoptosis and liver injury in mice livers.

Hepatic steatosis [by H\&E (40x magnification) and Oil Red O staining (20x magnification)], and apoptosis [by TUNEL staining (20x magnification)]. $\mathrm{C}=$ Control; $\mathrm{E}=$ Alcohol. 

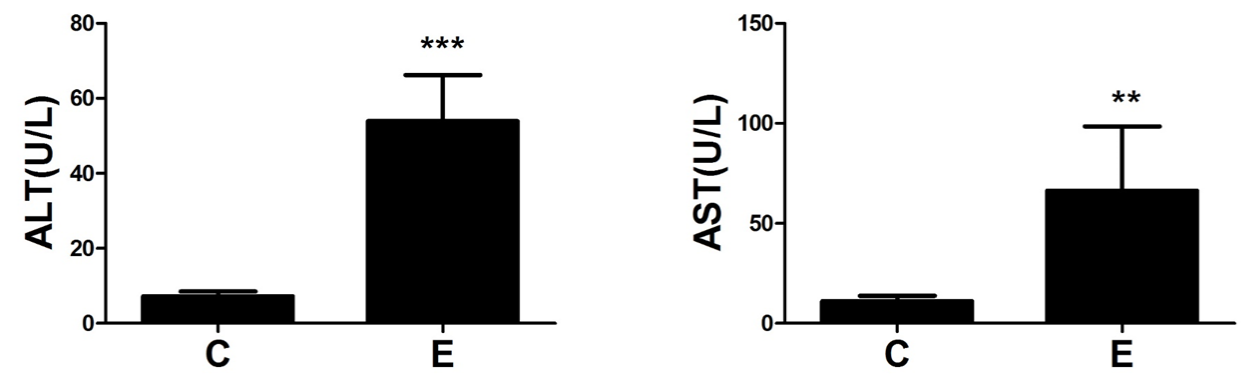

Figure 12B. Alcohol-induced acrolein and ER stress leads to steatosis, hepatocyte apoptosis and liver injury in mice livers.

Liver injury - serum ALT and AST. Mean \pm SEM, $n=6$ mice. ${ }^{* *} \mathrm{p}<0.01$ and ${ }^{* * *} p<0.001$ compared to control by Student's t-test. C=Control; E=Alcohol. 


\section{Acrolein mimics the in vivo effects of alcohol in cultured hepatic cells}

Along with acrolein, alcohol metabolism in the liver is capable of giving rise to many toxic metabolites including HNE and malondialdehyde. Hence, to isolate and determine the sole contribution of acrolein in alcohol-induced hepatic injury, we used H4IIEC cells to examine the direct in vitro effects of acrolein in comparison to alcohol, particularly pertaining to induction of ER stress and hepatocyte cell death. Similar to alcohol exposure, direct acrolein exposure of H4IIEC cells resulted in considerable acrolein adduct accumulation (Figure 13A). Further, analogous to alcohol exposure, direct in vitro acrolein exposure of hepatocytes resulted in ER stress and cell death. We exposed H4IIEC cells to variable concentrations of either alcohol $(50 \mathrm{mM}, 100 \mathrm{mM}$ or $200 \mathrm{mM})$ or acrolein $(20 \mu \mathrm{M}$ or $30 \mu \mathrm{M}$ ) for either $6 \mathrm{~h}$ (for mRNA) or $24 \mathrm{~h}$ (for protein). These concentrations of alcohol represent levels that may be encountered with moderate to high alcohol consumption in humans. The exact levels of acrolein that may be generated in the liver following alcohol consumption are difficult to predict, and the acrolein concentrations used here are based on published studies [77] and represent pathophysiological levels that caused hepatocyte apoptosis. Exposure of H4IIEC cells to either acrolein or alcohol yielded similar results; both triggered ER stress and increased ATF3 and ATF4, with minimal upregulation of GRP78 and GRP94, but a robust increase in proapoptotic CHOP (Figure 13B - mRNA and Figure 13C - protein). Further, consistent with the induction of $\mathrm{CHOP}$, we observed a corresponding loss of cell survival (Figure 13D). Thus, our data demonstrate that acrolein duplicates the effects of alcohol in cultured hepatic cells, indicating that 
the adverse effects of alcohol may be attributed to elevated acrolein in the liver occurring as a result of alcohol consumption. 

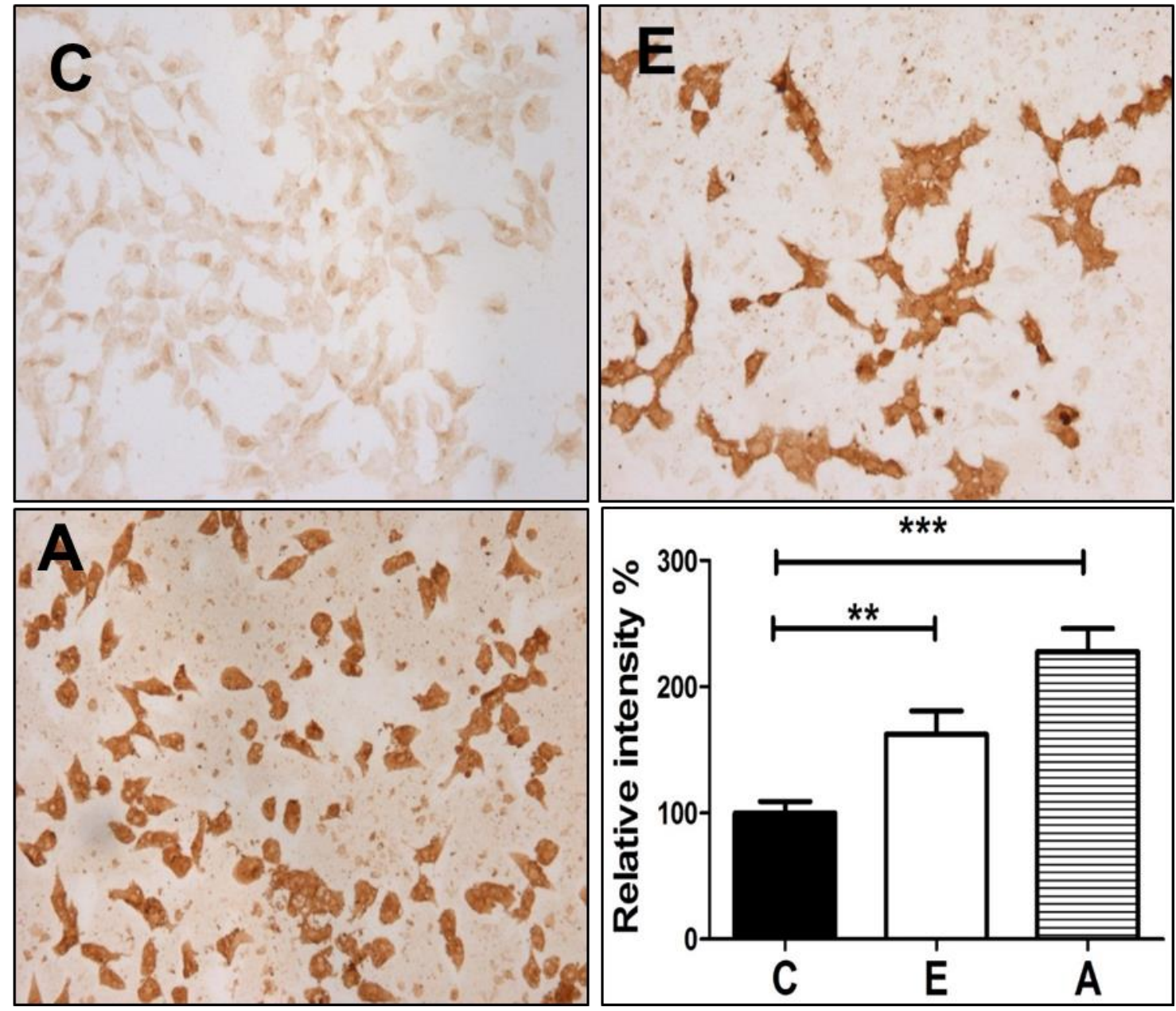

Figure 13A. Acrolein mimics the in vivo effects of alcohol and causes ER stress and cell death in cultured hepatic cells.

Accumulation and quantification of acrolein adducts (immunocytochemistry using specific FDP-lysine antibodies - 20x magnification) in H4IIEC cells treated for 24h. ${ }^{* *} p<0.01$ and ${ }^{* * *} p<0.001$ compared to control by ANOVA - Bonferroni analysis. $\mathrm{C}=$ control; $\mathrm{E}=200 \mathrm{mM}$ alcohol; and $\mathrm{A}=30 \mu \mathrm{M}$ acrolein . 


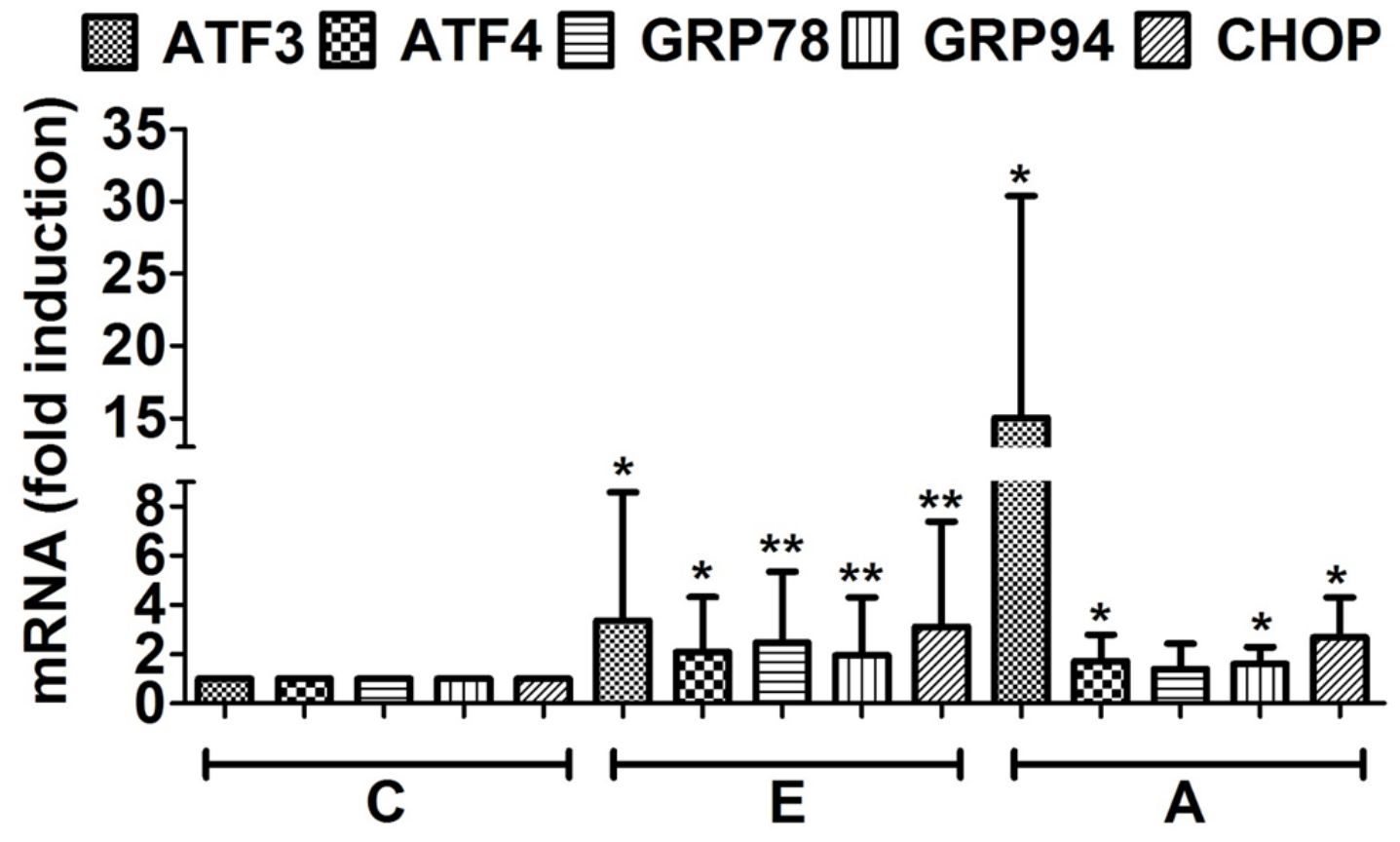

Figure 13B. Acrolein mimics the in vivo effects of alcohol and causes ER stress and cell death in cultured hepatic cells.

Total RNA was evaluated for mRNA expression ATF3, ATF4, GRP78, GRP94 and $\mathrm{CHOP}$ by real-time $\mathrm{PCR}$ at $6 \mathrm{~h}$ $\mathrm{C}=$ Control; $\mathrm{E}=$ alcohol $(200 \mathrm{mM}) ; \mathrm{A}=\mathrm{Acrolein}(30 \mu \mathrm{M})$. Mean $\pm \mathrm{SEM}, \mathrm{n}=3$ experiments. ${ }^{*} p<0.05$ and ${ }^{* *} p<0.01$ compared to control by ANOVA - Bonferroni analysis. 


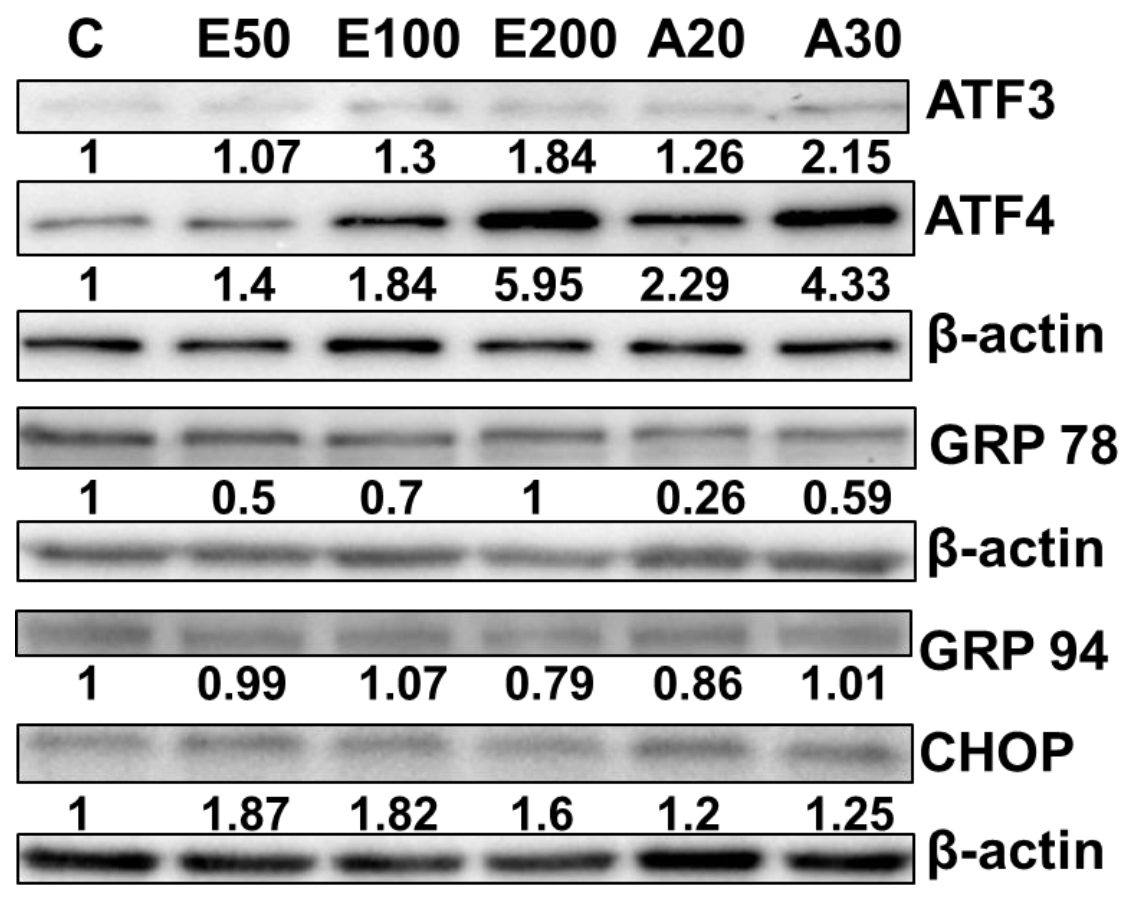

Figure 13C. ER stress gene expression ATF3, ATF4, GADD/CHOP, GRP78, and GRP94 by alcohol (E) or acrolein (A).

H4IIEC cells were treated with varying concentrations of ethanol $(50 \mathrm{mM}, 100 \mathrm{mM}$, and $200 \mathrm{mM})$ and acrolein $(20 \mu \mathrm{M}$ and $30 \mu \mathrm{M})$ for 24 hours. Western blot analysis was performed using total cell lysates. Blots were probed with ATF3, ATF4, GADD/CHOP, GRP78, and GRP94 antibodies, then stripped and reprobed with antibody to $\beta$-actin to ensure equivalent loading. Densitometry analysis was performed using Imagelab software. Density ratio was calculated using $\beta$-actin as control. Numbers represent mean density ratio for each treatment. 


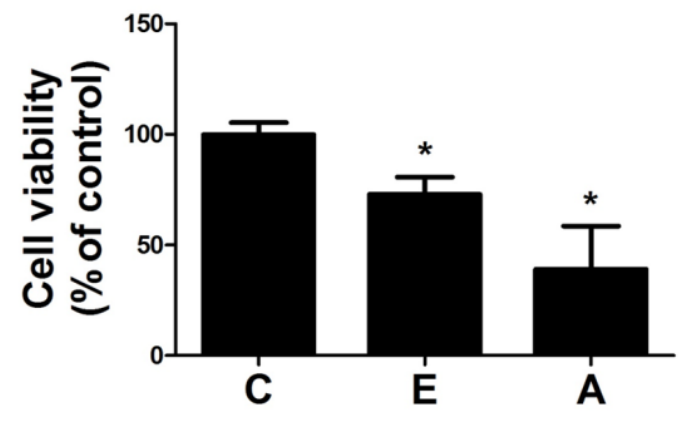

Figure 13D. Cell viability of cultured hepatocytes from alcohol $(E)$ or acrolein (A) toxicity.

Cell viability was measured by MTT assay as described in methods. E=Alcohol $200 \mathrm{mM}$ and $\mathrm{A}=$ acrolein $30 \mu \mathrm{M}$. Data are presented as the mean \pm SEM.

Statistical analysis was performed using GraphPad Prism Software using oneway ANOVA followed by Bonferroni posttest. ${ }^{*} p<0.05$ compared to Control $(n=3)$. 


\section{Acrolein scavengers exhibited protective effects both in vitro and in vivo}

In order to confirm the contribution of acrolein and establish its pathogenic role in alcohol-induced ER stress and liver injury, we tested known acrolein scavengers to mitigate the injurious effects of acrolein build-up. In this study, we used an acrolein scavenger, hydralazine, which is known to neutralize free acrolein $[78,79]$ and acrolein-protein adducts $[47,80]$, both of which are cytotoxic. Also, hydralazine was shown to effectively prevent acrolein-mediated cell death and tissue damage in spinal cord injury $[46,81]$. Additionally, we also used carnosine (beta-alanyl histidine, an endogenous dipeptide) that is known to scavenge aldehydes such as acrolein, and to have beneficial effects against acetaminophen-induced liver injury [82]. H4IIEC cells were pretreated with hydralazine or carnosine for $1 \mathrm{~h}$ prior to alcohol or acrolein exposure, and the effect on cell viability was monitored by the MTT assay. Acrolein- or alcohol-induced cell death was significantly attenuated by both acrolein scavengers, and hydralazine was slightly more effective (Figure 14A).

Finally, to firmly establish the role of acrolein in the development of ALD in vivo, we also tested the efficacy of hydralazine in scavenging acrolein and protecting against alcohol-induced liver injury in the NIAAA murine model of ALD. Hydralazine $(5 \mathrm{mg} / \mathrm{kg}$ body weight, dose based on previous literature $[79,81])$ was administered by daily intraperitoneal injection during the 10-day alcohol feeding regimen. As noted previously, consumption of the alcohol diet resulted in significant hepatic acrolein adduct accumulation and steatosis in the liver, and the acrolein scavenger hydralazine effectively blocked alcohol-induced acrolein formation and adduct accumulation (Figure 14B) and dramatically reduced hepatic 
steatosis as seen by histological examination and confirmed by Oil Red $O$ staining (Figure 14B). Further, alcohol-induced hepatic ER stress and transcriptional upregulation of hallmark ER stress mRNAs (ATF3, ATF4, CHOP and GRP78) were also substantially attenuated by hydralazine, most to near control levels (Figure 14C). Importantly, hydralazine prevented alcohol-induced proapoptotic signaling and activation of JNK, procaspase12 and CHOP (Figure 14D). A corresponding decrease was seen in alcohol-induced hepatic apoptosis as seen by decreased TUNEL positive cells (Figure 14E). Lastly, hydralazine showed marked protective effects against alcohol-induced liver injury with significantly reduced serum ALT and AST levels (Figure 14F). These data demonstrate that targeting and sequestration/neutralization of alcohol-induced hepatic acrolein accumulation by hydralazine administration significantly attenuated ER stress, apoptosis and liver injury, and protected against ALD in mice (Schematic - Figure 25). 


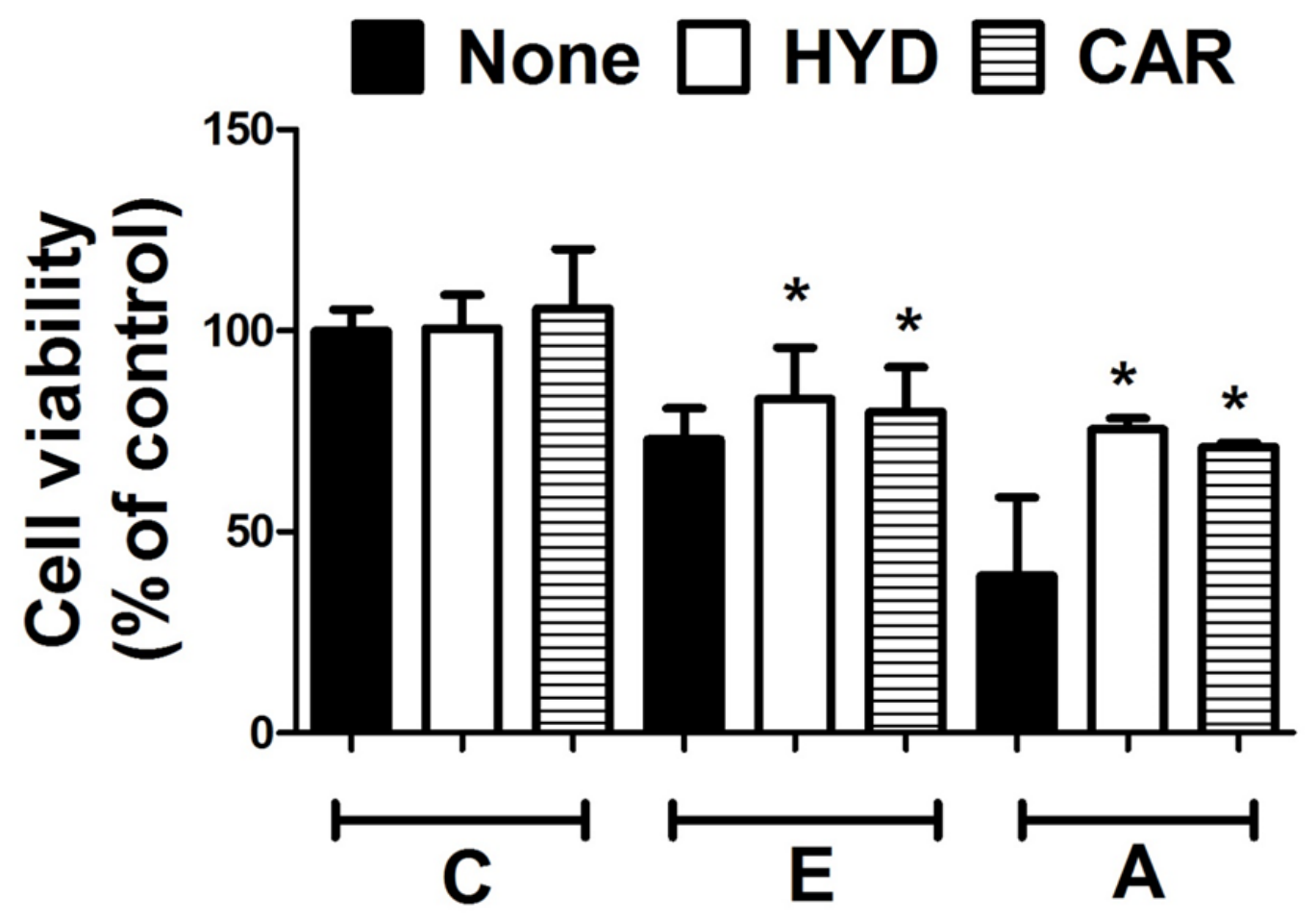

Figure 14A. Acrolein scavengers exhibit protective effects in vitro.

Cell survival in H4IIEC cells by MTT assay (24h). Mean \pm SEM, $n=3$ experiments.

${ }^{*} \mathrm{p}<0.05$ compared to the corresponding treatment of $E$ or A by ANOVA -

Bonferroni analysis. $\mathrm{C}=$ control; $\mathrm{E}=200 \mathrm{mM}$ alcohol; and $\mathrm{A}=30 \mu \mathrm{M}$ acrolein;

CAR=Carnosine; Hyd=Hydralazine. 

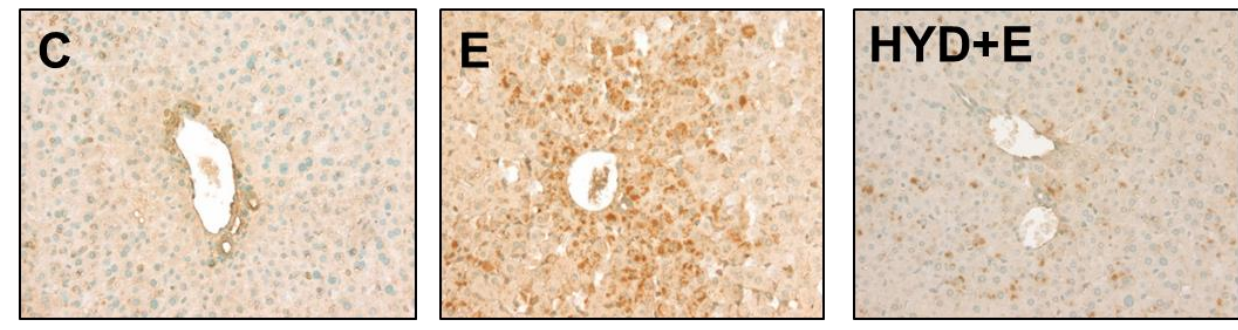

Acrolein
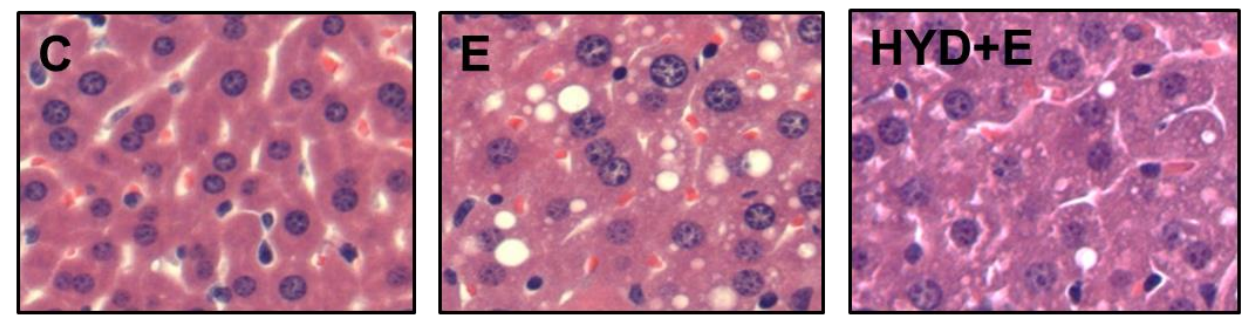
Adducts
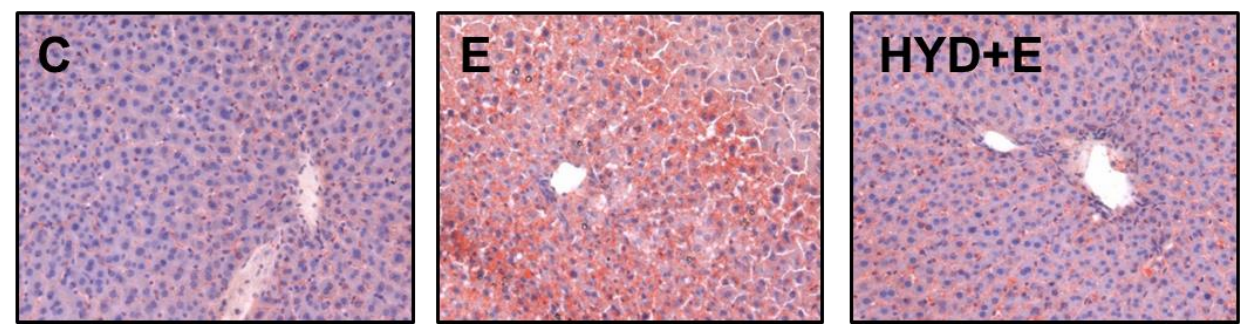

H\&E

Oil Red 0

Figure 14B. Acrolein scavengers exhibit protective effects in mice livers.

Hepatic acrolein adduct accumulation in mice (20x magnification), and hepatic steatosis by H\&E (80x magnification) and Oil Red O staining (20x magnification). $\mathrm{C}=$ Control; $\mathrm{E}=\mathrm{Alcohol} ; \mathrm{Hyd}+\mathrm{E}=$ Hydralazine+Alcohol . 


\section{$\square$ ATF3 囷 ATF4 因 GRP78 目 GRP94 $\square$ CHOP}

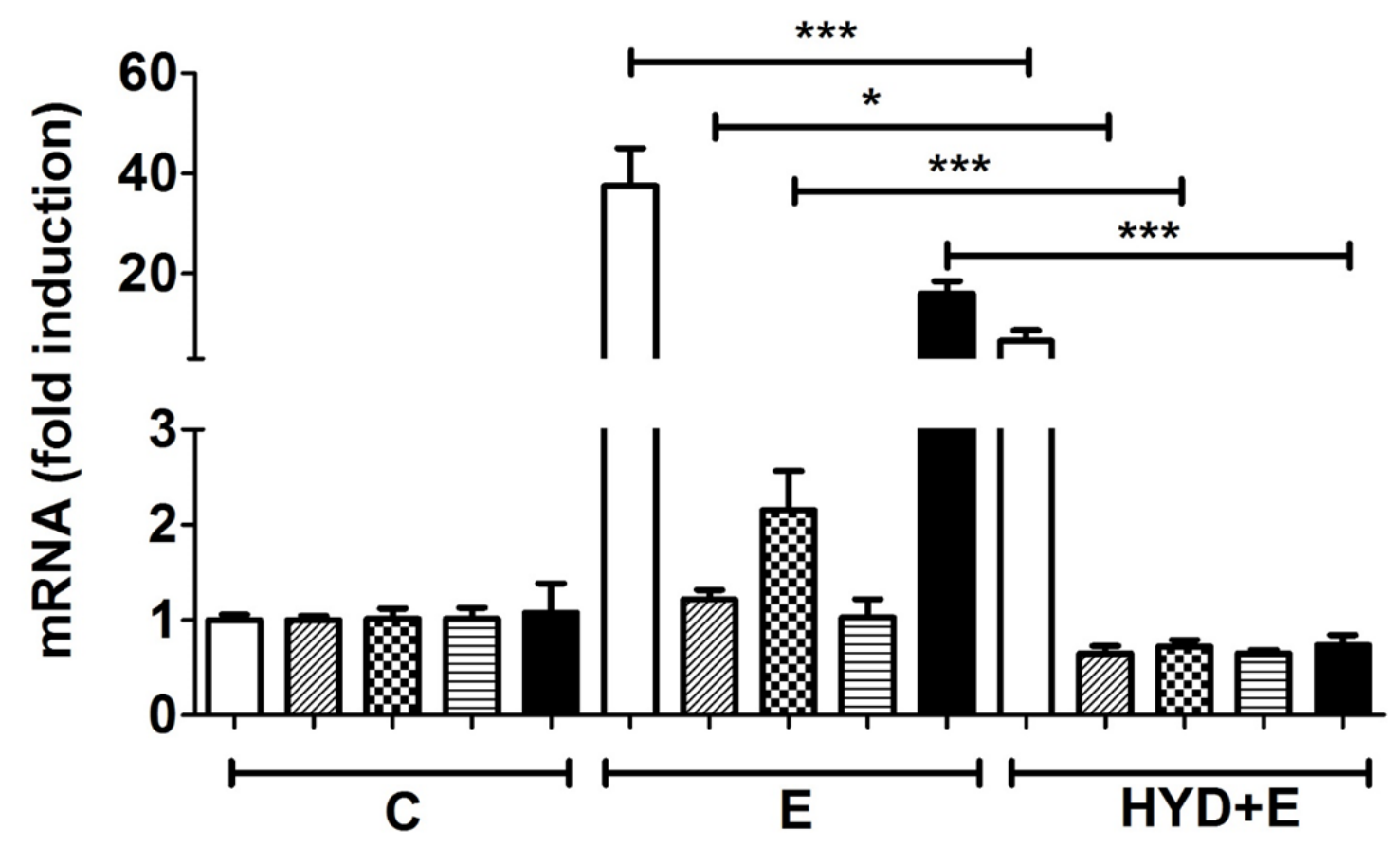

Figure 14C. Acrolein scavengers exhibit protective effects in mice livers.

ATF3, ATF4, GRP78, GRP94 and CHOP mRNA levels. Data are presented as mean \pm SEM ( $n=6$ mice). ${ }^{*} p<0.05$ and ${ }^{* * *} p<0.001$ compared to $E$ by ANOVA Bonferroni analysis. $\mathrm{C}=$ Control; $\mathrm{E}=\mathrm{Alcohol}$; $\mathrm{Hyd}+\mathrm{E}=\mathrm{Hydralazine+Alcohol}$. 


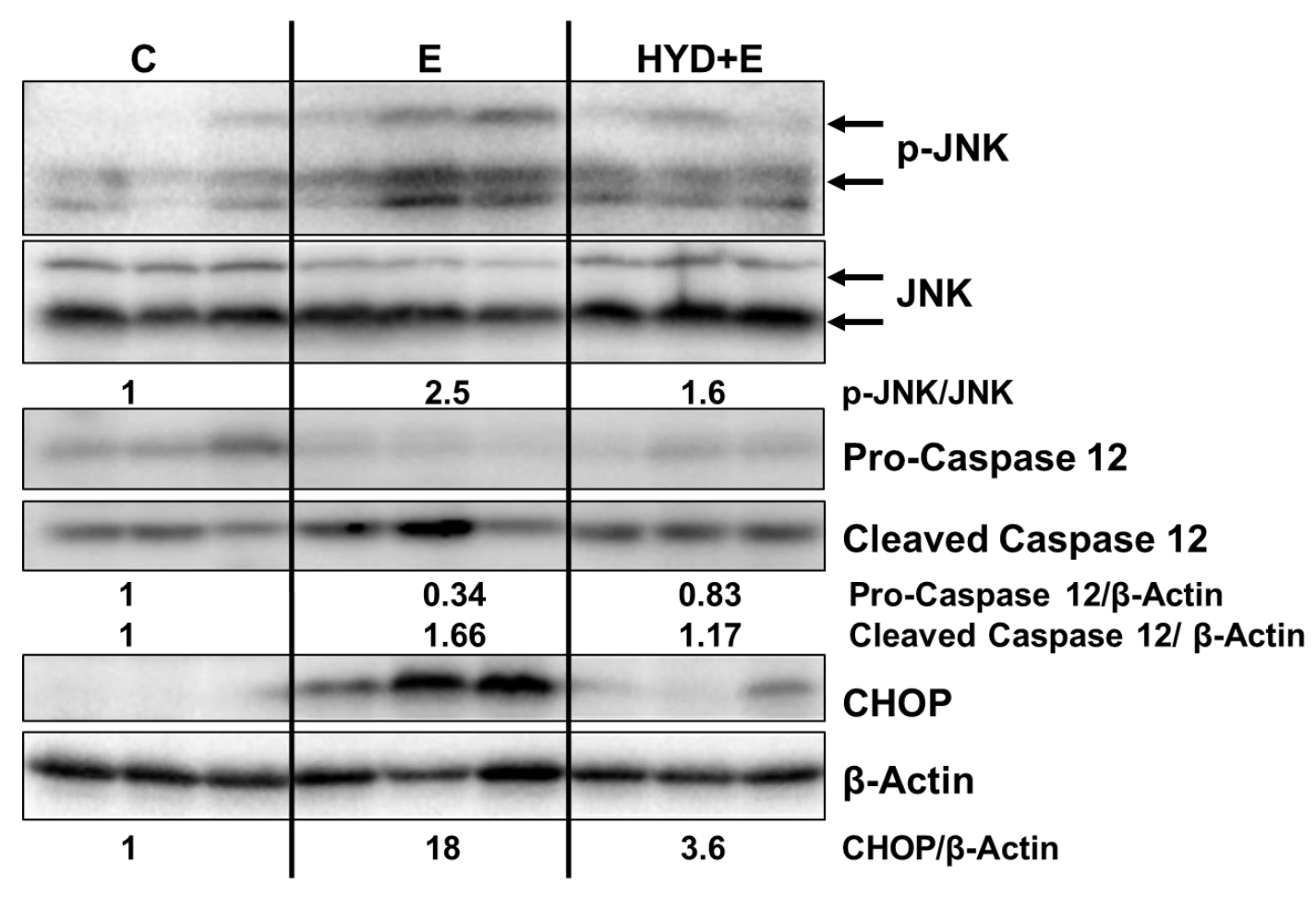

Figure 14D. Acrolein scavengers exhibit protective effects in mice livers.

Protein levels of phospho-JNK and total JNK, pro- and cleaved/active caspase 12, and CHOP. Numbers represent mean densitometry ratios normalized to corresponding control proteins (total JNK or $\beta$-actin). $\mathrm{C}=\mathrm{C}$-ontrol; $\mathrm{E}=\mathrm{Alcohol}$; $\mathrm{Hyd}+\mathrm{E}=$ Hydralazine + Alcohol . 


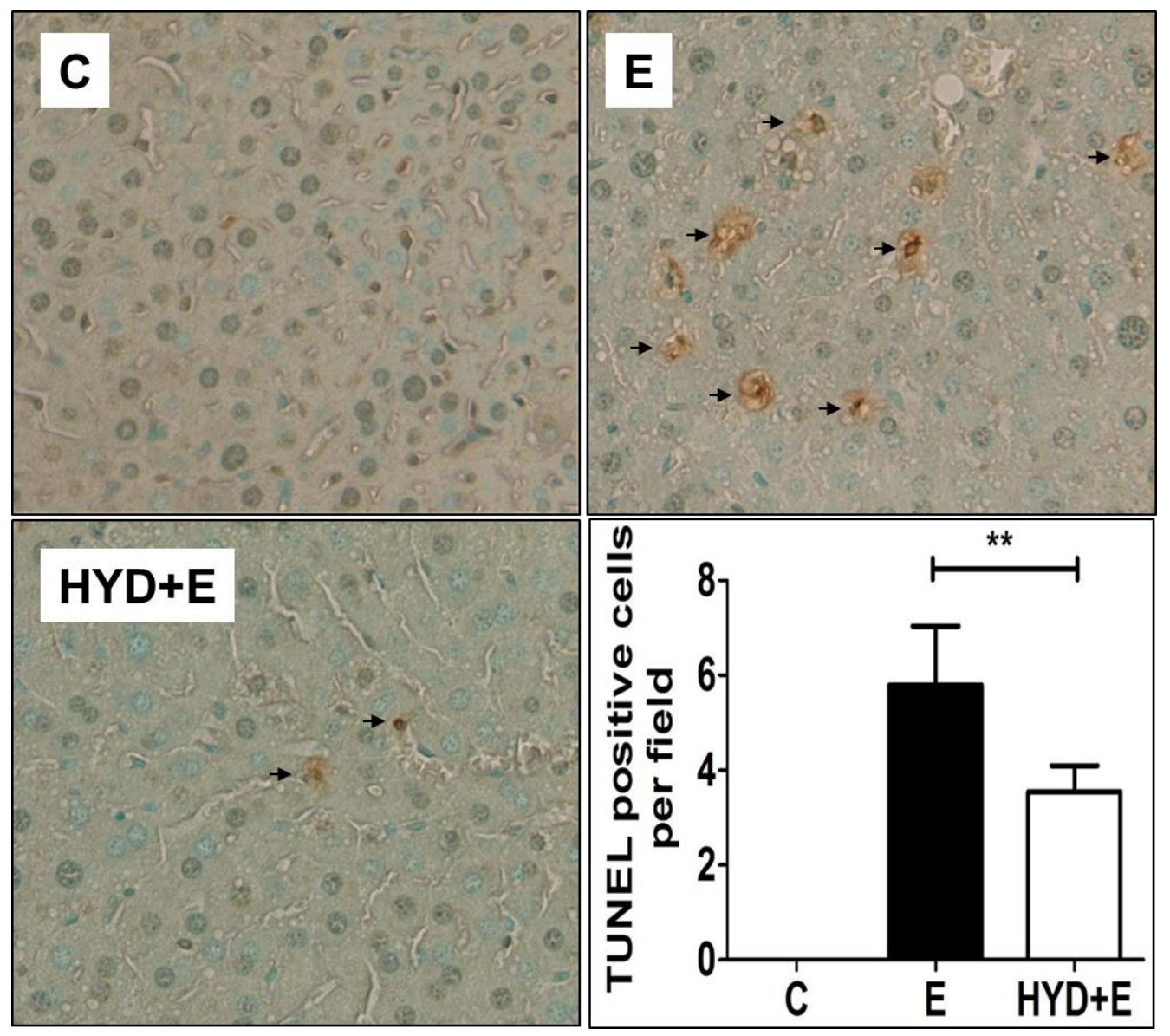

Figure 14E. Acrolein scavengers exhibit protective effects in mice livers.

Apoptosis by TUNEL staining (80x magnification), with quantification of apoptosis. ${ }^{* *} \mathrm{p}<0.01$ compared to $\mathrm{E}$ by ANOVA - Bonferroni analysis. $\mathrm{C}=\mathrm{Control}$; $\mathrm{E}=$ Alcohol; Hyd $+\mathrm{E}=$ Hydralazine+Alcohol. 

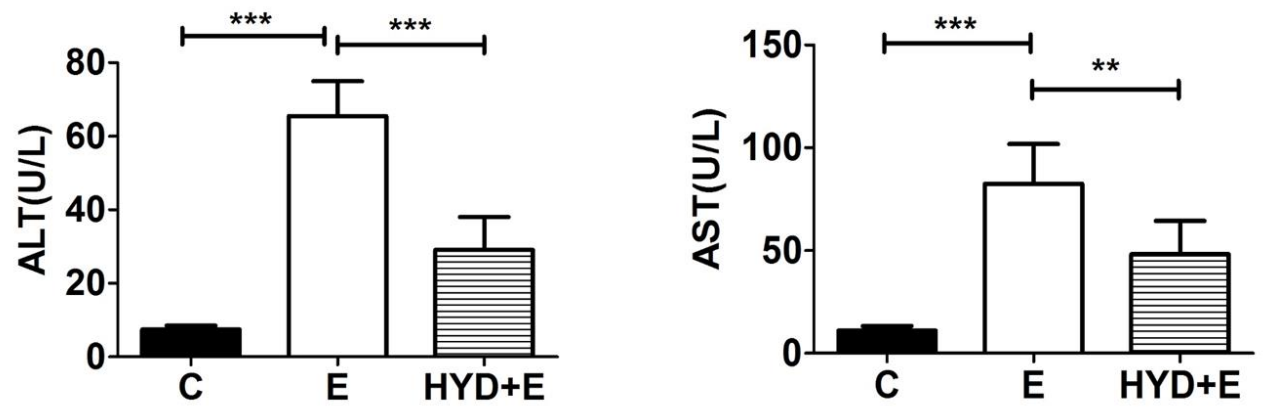

Figure 14F. Acrolein scavengers exhibit protective effects in mice livers.

Liver injury - serum ALT and AST. Mean \pm SEM, $n=6$ mice. ${ }^{* *} p<0.01$ and ${ }^{* * *} \mathrm{p}<0.001$ by ANOVA - Bonferroni analysis. $\mathrm{C}=$ Control; $\mathrm{E}=\mathrm{Alcohol}$;

$\mathrm{Hyd}+\mathrm{E}=$ Hydralazine + Alcohol . 


\section{RESULTS SECTION II.}

Increasing evidence suggests that interactions between the intestine and liver (gutliver axis) play a critical role in the development/progression of ALD [49].

In the Results Section I above, we discussed the contributory role of acrolein to the hepatic aspect of alcohol-induced liver injury. This section describes the intestinal effects of acrolein in ALD using cultured human intestinal epithelial cells (Caco-2), and the chronic+binge murine model of ALD.

\section{Alcohol consumption leads to intestinal acrolein generation, accumulation of acrolein adducts and serum endotoxemia}

Alcohol consumption causes lipid peroxidation and leads to acrolein adducts accumulation. In order to validate our hypothesis that acrolein disrupts the intestinal epithelial barrier leading to increased gut permeability, we first examined whether alcohol consumption in mice led to acrolein buildup in the intestine. The ileum was investigated since it is critical for nutrient absorption, and it has been demonstrated to be affected under pathological disease conditions, such as ulcerative colitis and alcoholic liver disease [83]. From our data, a marked increase was observed in the levels of acrolein-protein adducts (brown staining of acroleinFDP-lysine adducts) in the ileum of alcohol-fed mice (Figure 15) compared to controls, showing that alcohol consumption led to the intestinal generation/accumulation of acrolein. The alcohol-induced acrolein adduct accumulation was largely in the epithelium, lacteal, and lamina propria. Hydralazine, an acrolein scavenger, which effectively trapped acrolein-protein adducts, lowered acrolein adduct accumulation in the ileum. These data show that 
hydralazine can reduce the accumulation of acrolein protein adducts in the ileum of alcohol fed animals.

In order to test if the clearance of acrolein-protein adducts by hydralazine improves intestinal barrier function, we examined the level of endotoxin in the serum of the mice. An elevated LPS level was detected in the alcohol-fed mice compared to control (Figure 16) which indicated disruption of intestinal barrier function. In our previous liver data, hydralazine, the acrolein scavenger, showed a protective effect against alcohol-induced ER stress and liver injury. Interestingly, the increased LPS level was significantly attenuated by hydralazine in the serum compared to the alcohol group. These data demonstrate that alcohol causes intestinal barrier dysfunction and leads to increased gut permeability which, in turn, allows elevated LPS levels in the serum. Hydralazine, which is known to neutralize free acrolein $[78,79]$ and acrolein-protein adducts $[47,80]$, exhibits a protective effect in alcohol induced endotoxemia. Thus, hydralazine, the acrolein scavenger, attenuated alcohol induced endotoxemia. 

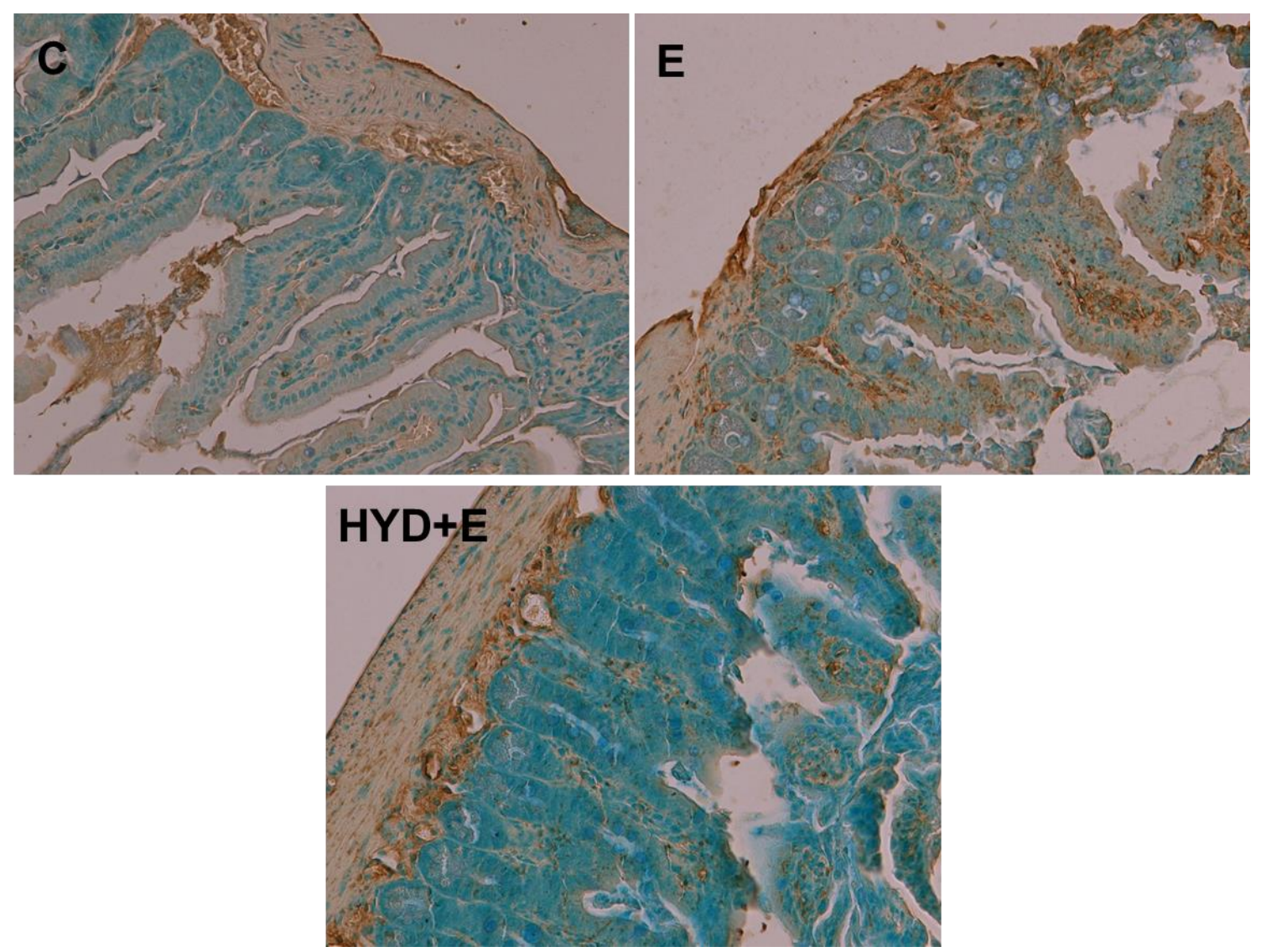

Figure 15: Exposure to alcohol causes acrolein adduct buildup in the ileum of mice.

Accumulation of acrolein adducts in mice ileum by immunohistochemistry using specific FDP-lysine antibodies (20x).

$\mathrm{C}=$ Control; $\mathrm{E}=$ Ethanol; HYD+E=Hydralazine+ethanol. 


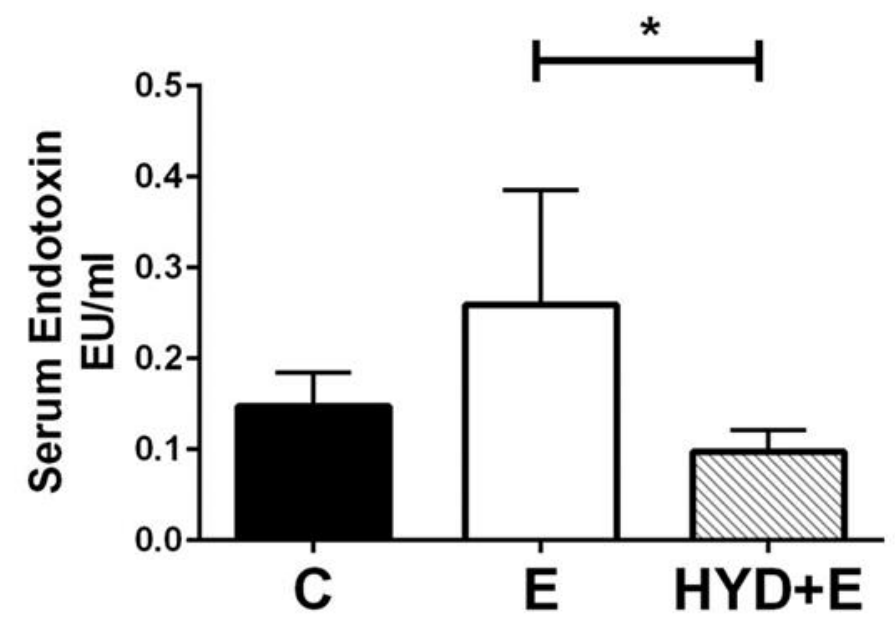

Figure 16: Hydralazine attenuated alcohol-induced serum endotoxemia.

Serum LPS levels. Data are presented as mean \pm SEM ( $n=6$ mice). ${ }^{*} p<0.05$ compared to $\mathrm{E}$ by ANOVA - Bonferroni analysis. $\mathrm{C}=\mathrm{Control}$; $\mathrm{E}=\mathrm{Ethanol}$; HYD+E=Hydralazine+ethanol. $(n=6)$. 


\section{Caco-2 cells were used to study the involvement of acrolein in the intestine}

in ALD.

From our animal data, we saw accumulation of acrolein protein adducts in the ileum and a corresponding rise of serum endotoxemia was observed in the alcoholfed mice. In order to mimic the in vivo acrolein effect on the intestine, the direct effects of acrolein were investigated using a well characterized and accepted model of the intestine: murine cultured intestinal epithelial Caco-2 cells, which form a polarized confluent monolayer that mimics the intestinal epithelium [66]. Cells were grown for 21 days to form epithelial monolayers and were then treated with alcohol or acrolein to compare their effects. Acrolein adduct accumulation was seen in Caco- 2 cells that were treated for $24 \mathrm{~h}$ with alcohol $(200 \mathrm{mM})$ or acrolein (20uM) compared to untreated cells (Figure 17). To determine whether acrolein adduct accumulation affected the Caco-2 epithelial barrier, we measured intestinal barrier function and permeability using Trans Epithelial Electrical Resistance (TEER) and FD-4 leakage, respectively. Alcohol treatment for $6 \mathrm{~h}$ caused a significant decrease in the epithelial TEER measurements in a dose-dependent manner. Consistent with these results, the paracellular permeability to FD-4 was significantly increased by alcohol exposure in a dose-dependent manner (Figure 18). Similar to alcohol, direct acrolein exposure for $6 \mathrm{~h}$ also caused a significant decrease in the epithelial TEER measurements, and a corresponding increase in paracellular permeability to FD-4 (Figure 19). These data indicated that alcohol or alcohol induced acrolein protein adducts promote disruption of intestinal barrier 
function and an increase in intestinal barrier permeability with increasing concentrations of alcohol or acrolein. 

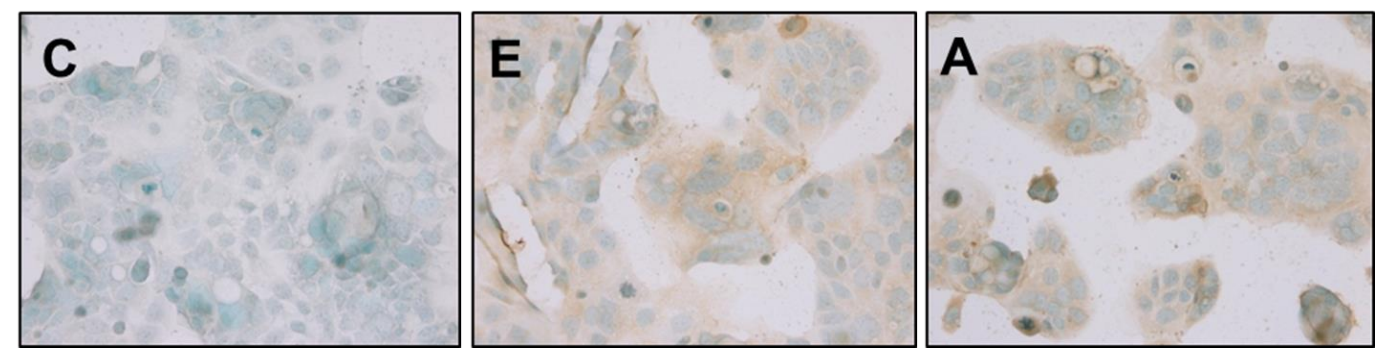

Figure 17: Exposure to alcohol or acrolein leads to acrolein adduct accumulation in Caco-2 cell monolayers.

Accumulation of acrolein adducts by immunocytochemistry using specific FDPlysine antibodies (20x magnification) in Caco-2 cells treated for $24 \mathrm{~h}$ as follows: $\mathrm{C}=$ Control; E=Ethanol $(200 \mathrm{mM}) ; \mathrm{A}=$ Acrolein $(20 \mu \mathrm{M})$. 

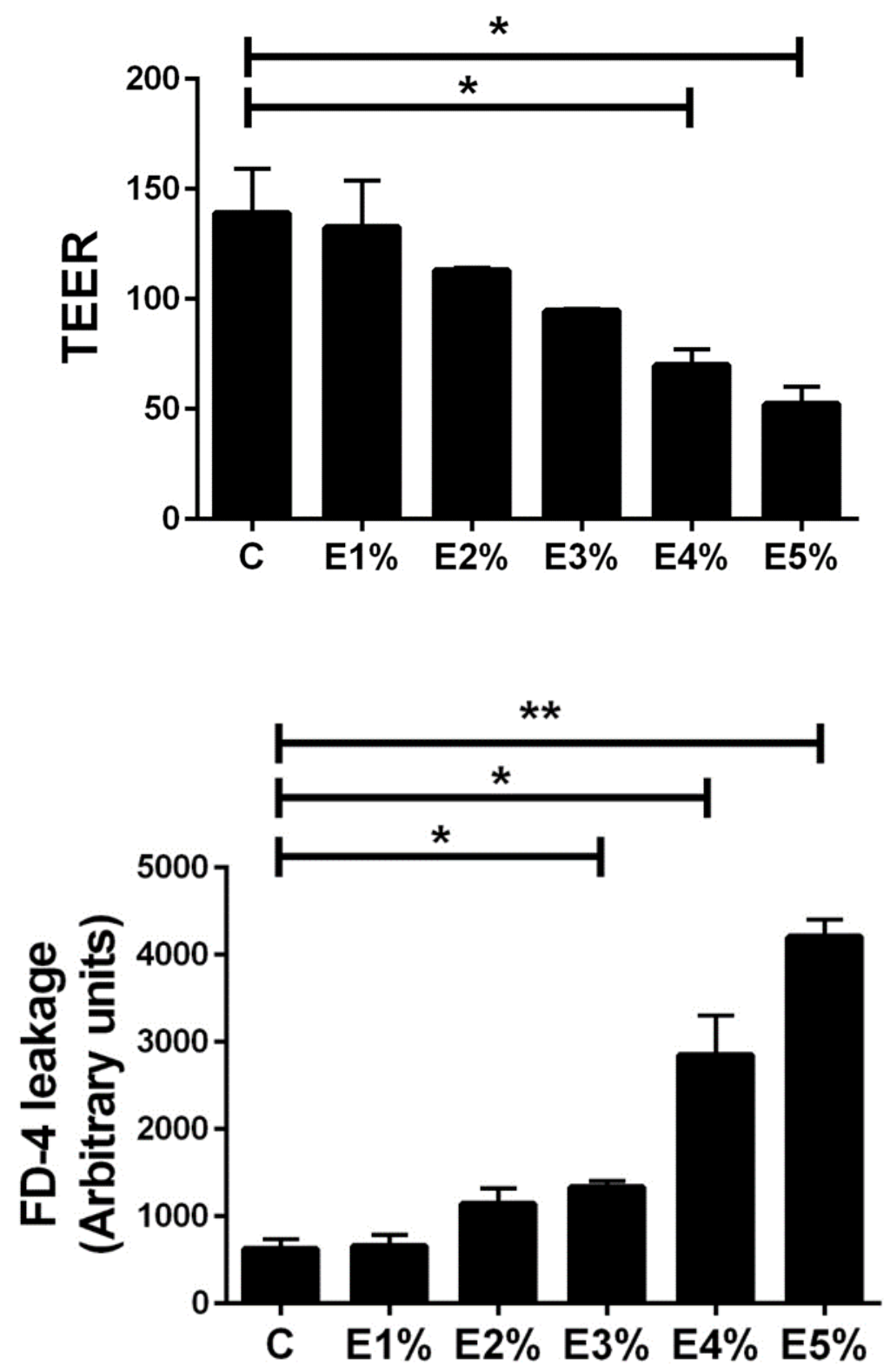

Figure 18. Alcohol exposure decreases barrier function and increases permeability (FD-4) (TEER) Caco-2 cells.

$\mathrm{C}=$ Control; $\mathrm{E}=\mathrm{Ethanol}(\%)$ Data are presented as the mean \pm SEM. Statistical analysis was performed using GraphPad Prism Software using one-way ANOVA followed by Bonferroni posttest. ${ }^{*} p<0.05$ and ${ }^{* *} p<0.01$ compared to Control $(n=3)$. 

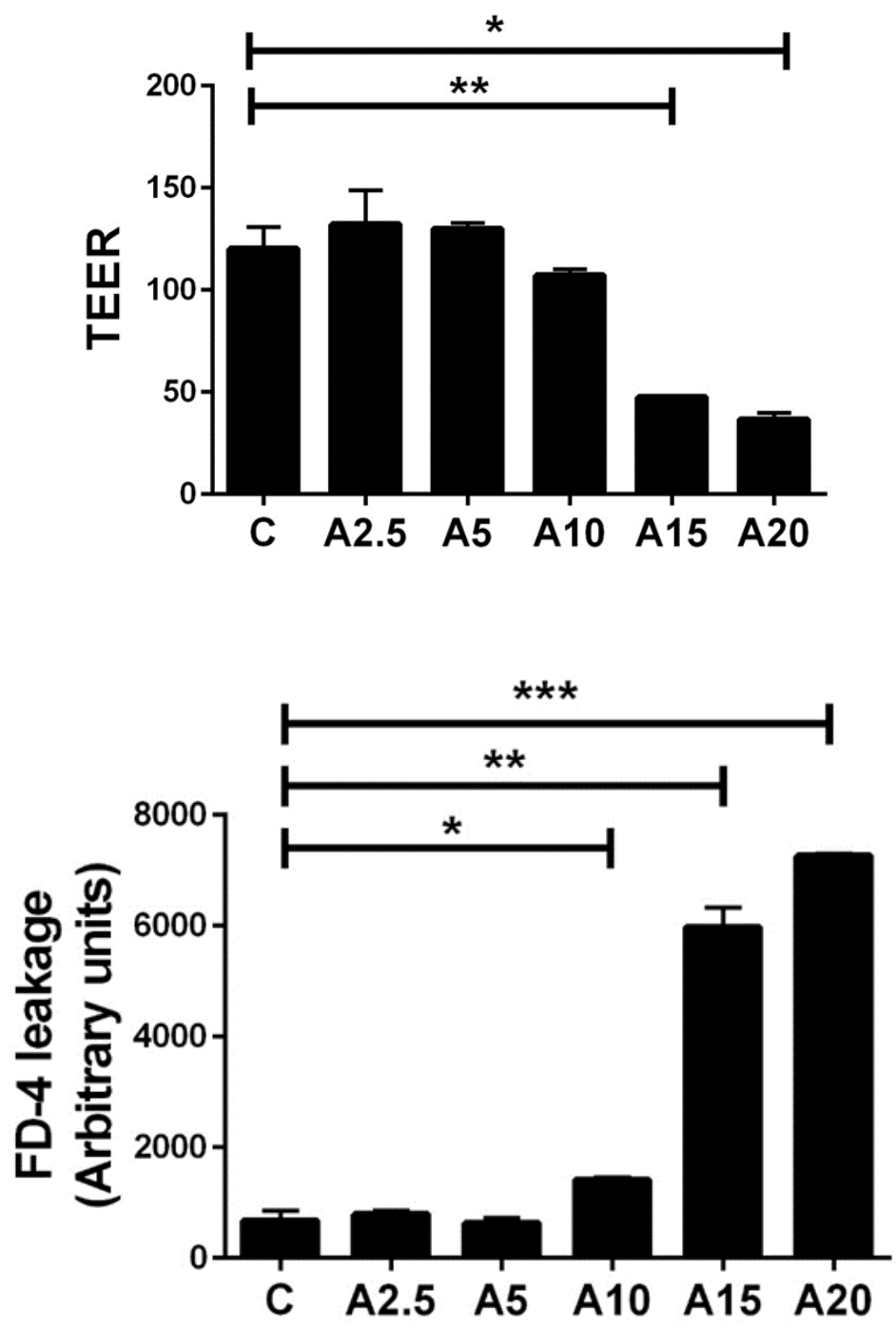

Figure 19. Acrolein exposure decreases and increases permeability (FD-4) barrier function (TEER) in Caco-2 cells.

$\mathrm{C}=$ Control; $\mathrm{A}=$ Acrolein $(\mu \mathrm{M})$. Data are presented as the mean \pm SEM. Statistical analysis was performed using GraphPad Prism Software using one-way ANOVA followed by Bonferroni posttest. ${ }^{*} p<0.05,{ }^{* *} p<0.01$ and ${ }^{* *} p<0.001$ compared to Control $(n=3)$. 


\section{Alcohol or acrolein exposure leads to down regulation and redistribution of TJPs}

Tight junction proteins (TJP; e.g., ZO-1, claudin-1 and occludin) are critical components of the intestinal barrier. ZO-1 is the tight junction scaffolding protein and it has been reported that reduced level of ZO-1 leads to disruption of the intestinal mucosa and an increase in permeability after intestinal ischemiareperfusion injury [84]. Claudin-1 plays an important role in the maintenance of gut integrity. Decreased claudin-1 leads to increased intestinal permeability while overexpressed claudin-1 reduces paracellular permeability and maintains the integrity of intestinal barrier [85]. We demonstrated that alcohol exposure downregulated TJP mRNAs (ZO-1, occludin and claudin-1) from 3\% to 5\% (Figure 20) in 24 hours. Similar to alcohol treatment, the treatment of acrolein at 10 to 20 uM also showed downregulation of TJP mRNAs (ZO-1, occludin and claudin-1) (Figure 19) in 24 hours. The effect of acrolein (10 and $20 \mathrm{uM}$ ) on the protein levels of TJPs (ZO-1, occludin, claudin-1) was also evaluated (Figure 21). The effects on total protein levels were variable; claudin-1 and occludin were downregulated by acrolein, in agreement with the decrease in mRNA levels, but ZO-1 was increased slightly (Figure 21). Since the location and membrane association of the TJPs is critical for their function, any alterations in their localization can greatly impact the regulation of barrier permeability. Hence, we examined the protein levels in the membrane-bound (insoluble in detergent) and the soluble, or nonmembrane associated, fractions (Figure 22). Acrolein treatment resulted in a dramatic increase in the soluble protein levels of all three TJPs, indicating that their 
membrane association was significantly disrupted. We observed a corresponding decrease in the insoluble levels of both ZO-1 and claudin, but not occludin (Figure 22).

To further support these findings and to examine TJP redistribution in whole cells rather than extracted protein samples, we used immunofluorescence microscopy to assess the localization of ZO-1 (as a representative TJP) upon exposure to alcohol or acrolein. Both alcohol (2\% or $5 \%$ ) and acrolein (10 and 20 $\mu \mathrm{M})$ showed significant and similar dose-related effects on the distribution pattern of ZO-1 (Figure 23). Compared with the untreated control cells which exhibited a smooth regular pattern of ZO-1 staining at the cell borders between the adjacent epithelial cells, cells treated with either acrolein or alcohol displayed an uneven (ruffled) distribution. ZO-1 was decreased and discontinuous at some locations, while a random accumulation and clumping of ZO-1 was seen in other cells.

Overall, our data showed that alcohol or acrolein exposure caused the downregulation and/or redistribution of TJPs, leading to consequent intestinal barrier dysfunction and increased permeability. 


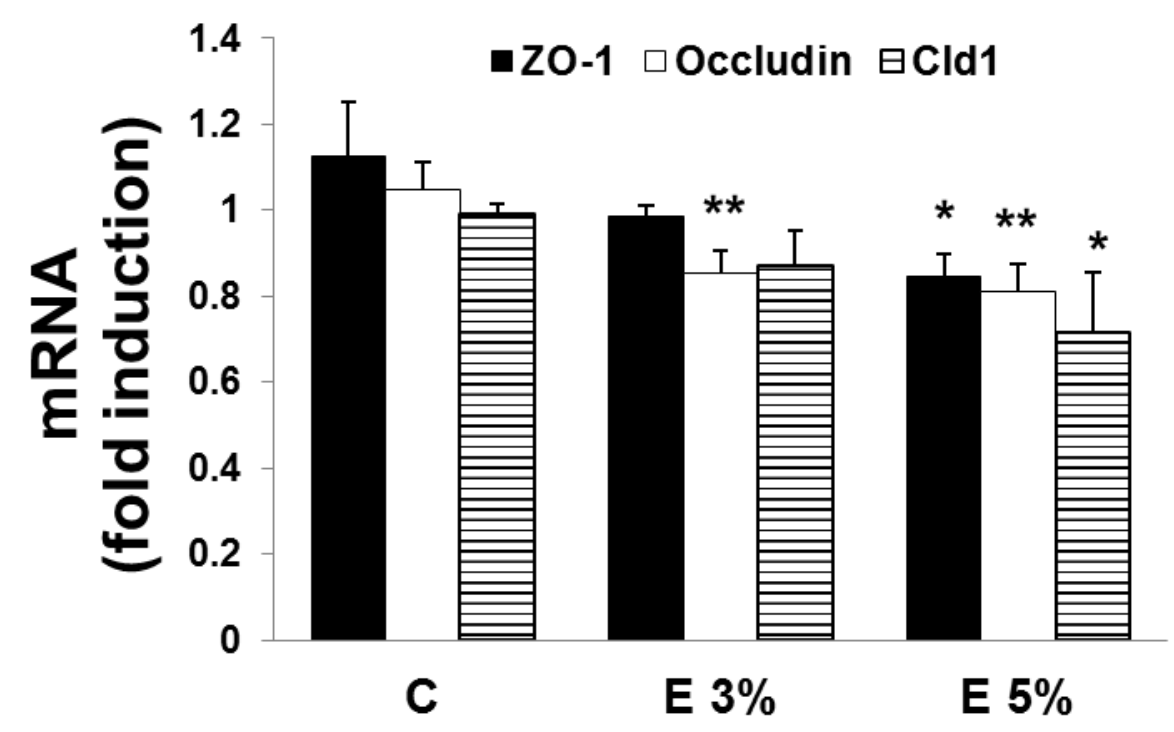

Figure 20. In vitro alcohol exposure down-regulates TJP mRNAs. (Real time qPCR).

Total RNA was evaluated for mRNA expression ZO-1, Occludin, and Claudin in Caco-2 cells treated for $24 \mathrm{~h}$.

$\mathrm{C}=$ Control; $\mathrm{E}=$ Alcohol. Mean $\pm \mathrm{SEM}, \mathrm{n}=3$ experiments. ${ }^{*} \mathrm{p}<0.05$ and

${ }^{* *} \mathrm{p}<0.01$ compared to control by ANOVA - Bonferroni analysis. 


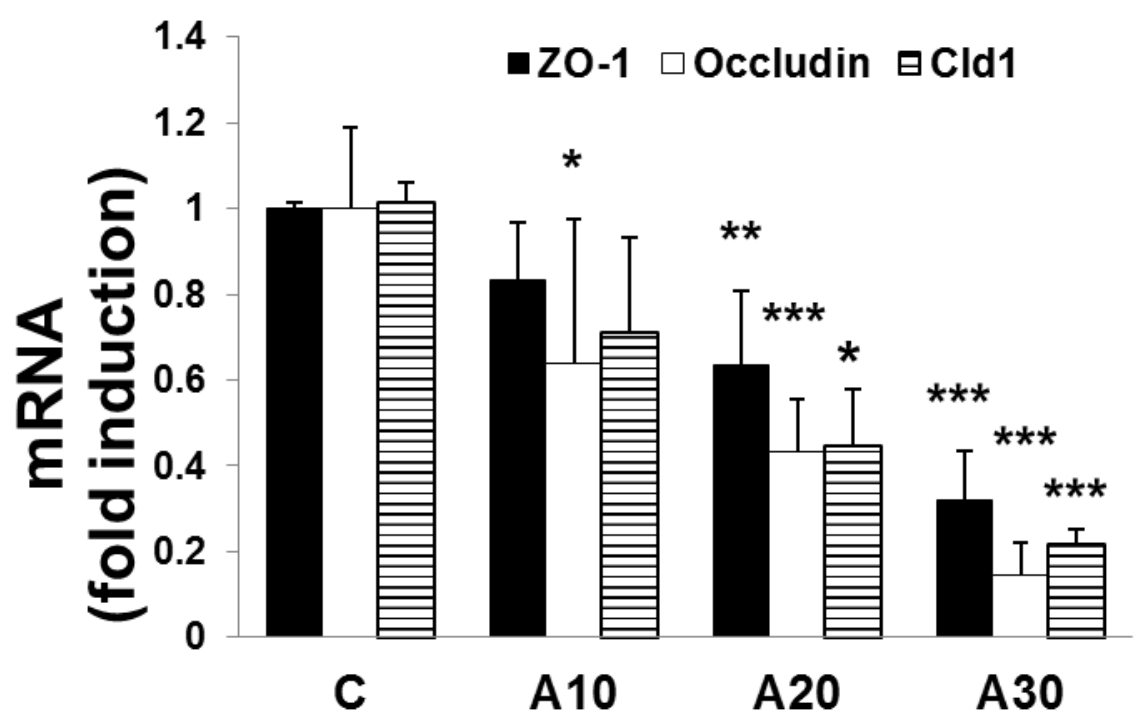

Figure 21. In vitro acrolein exposure down-regulates TJP mRNAs. (Real time qPCR).

Total RNA was evaluated for mRNA expression ZO-1, Occludin, and Claudin in Caco-2 cells treated for $24 \mathrm{~h}$.

$\mathrm{C}=$ Control; $\mathrm{A}=$ Acrolein. Mean $\pm \mathrm{SEM}, \mathrm{n}=3$ experiments. ${ }^{*} \mathrm{p}<0.05,{ }^{* *} \mathrm{p}<0.01$, and ${ }^{* * *} \mathrm{p}<0.001$ compared to control by ANOVA - Bonferroni analysis. 

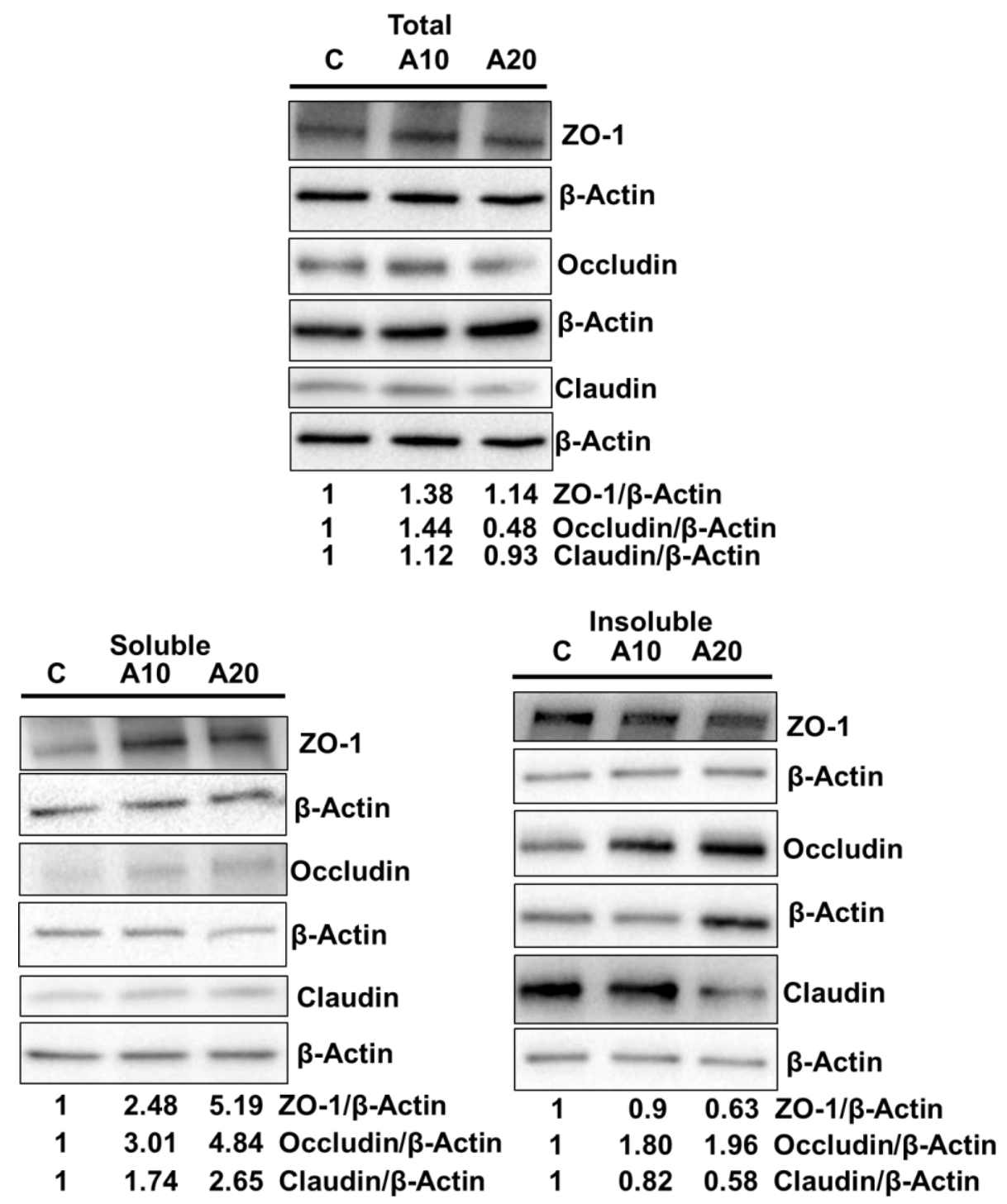

Figure 22. Acrolein exposure alters protein levels of TJP.

Western blot analysis of ZO-1, Occludin, and Claudin were performed using tight junction total, soluble, and insoluble protein lysates. Blots were reprobed with antibody to $\beta$-actin to ensure equivalent loading. Densitometry analysis was performed using Imagelab software. Density ratio was calculated using $\beta$-actin as control. Numbers represent the average density ratio for each treatment. $\mathrm{C}=$ Control; $\mathrm{A}=$ Acrolein $(\mathrm{UM})$. 

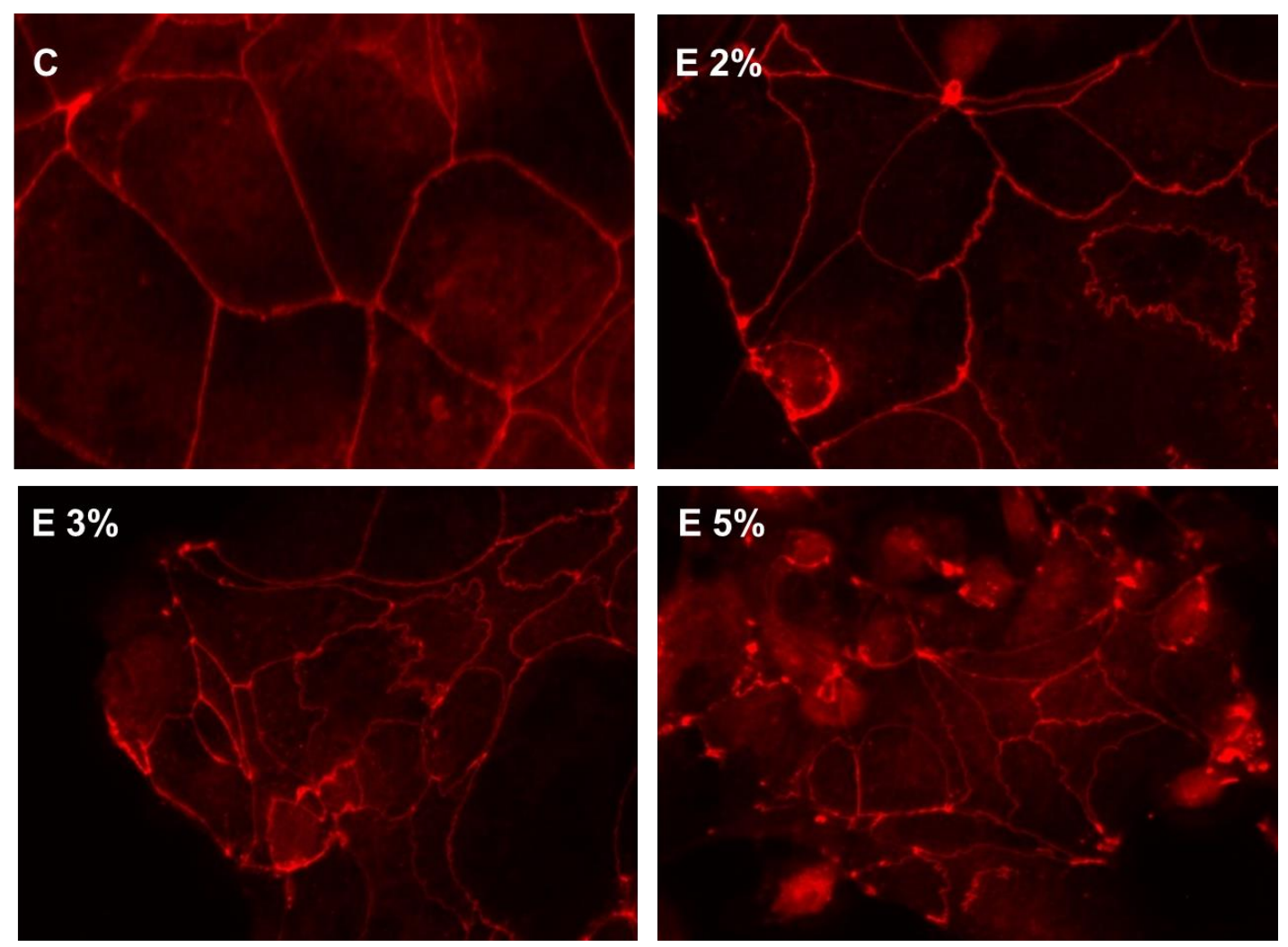

Figure 23. Alcohol treatment causes disrupted distribution of ZO-1 protein in Caco-2.

Caco2 cells were treated with or without alcohol for 24 hours, and ZO-1 protein localization was assessed by immunofluorescent microscopy. Results are representative of 3 experiments. (magnification, 40X). $\mathrm{C}=\mathrm{C}$ Control; $\mathrm{E}=\mathrm{Alcohol}(\%)$. 


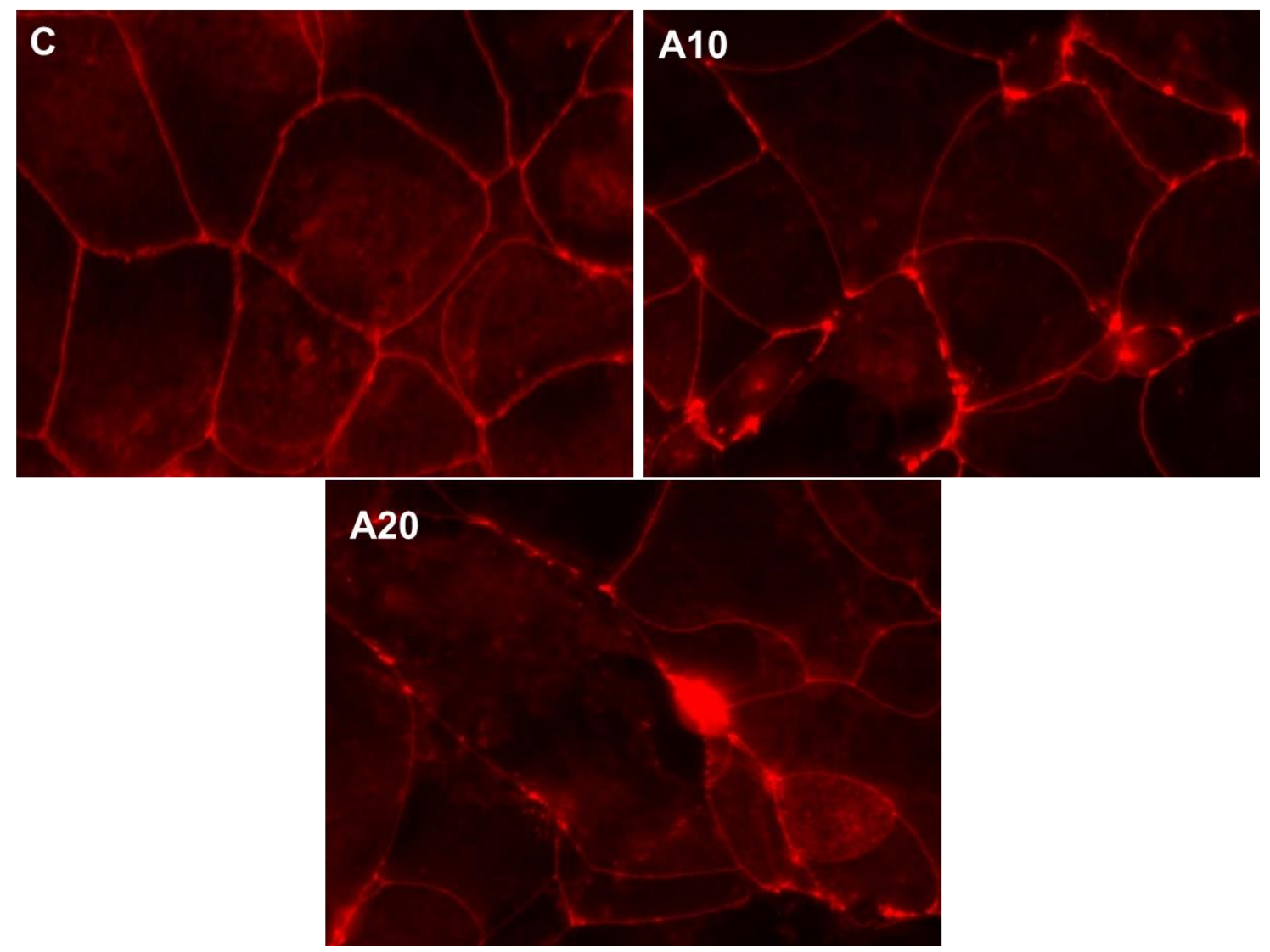

Figure 24. Acrolein treatment causes disrupted distribution of ZO-1 protein in Caco-2.

Caco2 cells were treated with or without acrolein for 24 hours, and ZO-1 proteins localization was assessed by immunofluorescent microscopy. Results are representative of 3 experiments. (magnification, 40X). $\mathrm{C}=\mathrm{Control}$; $\mathrm{A}=\mathrm{Acrolein}$ $(\mu \mathrm{M})$. 


\section{CHAPTER 4}

\section{DISCUSSION}

Alcohol consumption is a major health problem worldwide and in the USA; it has no FDA-approved therapy for any stage of ALD. Therefore, it is critical to investigate the mechanisms involved in ALD, and to identify novel therapeutic targets and strategies for the prevention and treatment of ALD. In this study, we pursued in vitro and in vivo studies to demonstrate the role of the lipid peroxidation byproduct, acrolein, in contributing to alcohol-induced gut barrier dysfunction and hepatic ER stress, steatosis, cell death and liver injury in the pathogenesis of gutliver injury of ALD. Our study demonstrates that: (i) alcohol consumption generates hepatic acrolein, triggers pathological ER stress and hepatocyte apoptosis, with insufficient activation of ER-adaptive/protective responses; (ii) alcohol-induced acrolein is a major mediator of hepatic ER stress, cell death and injury, and is a potential therapeutic target in ALD; (iii) removal/clearance of acrolein by scavengers has therapeutic potential in ALD; and (iv) alcohol consumption generates intestinal acrolein, down-regulates tight junction proteins, alters their localization, and disrupts intestinal barrier function (Figure 25). Acrolein is formed both endogenously from lipid peroxidation and exogenously from environmental and dietary exposures such as air pollution, cigarette smoking, automobile exhausts, charred meat, and fried foods. The accumulation of acrolein can occur 
and cause problems in other diseases that involve increased lipid peroxidation and oxidative stress.

Our hepatic results demonstrated significant generation/accumulation of acrolein in the liver following alcohol exposure resulting in cytoplasmic and nuclear accumulation of acrolein adducted proteins. The accumulation of acrolein-protein adducts triggered subsequent hepatic ER stress leading to hepatocyte cell death and liver injury. Notably, all three ER stress pathways involving the ER sensors PERK, IRE1 and ATF6, were activated; however, not all ER stress genes were affected in the same way. Alcohol consumption appeared to trigger ER stress and induce apoptotic signaling (JNK, Caspase12 and CHOP), while simultaneously suppressing UPR-protective responses (XBP1 splicing and ER chaperones (GRP78 and 94)). The differential regulation of ER stress genes may be via altered signal transduction, or by direct or indirect protein adduction. Also, the nuclear accumulation of acrolein adducts may indicate adduction and alteration of function of transcription factors or chromatin-modifying proteins such as histone deacetylases and DNA/histone methylases, which likely have significant effects on downstream gene expression. The exact mechanisms by which acrolein adduct accumulation causes ER stress, and the identity and functionality of the proteins adducted by acrolein are currently under investigation.

Oxidative stress is known to induce ER stress, however, the mediators responsible for such effects have not been clearly identified. Alcohol-induced oxidative stress and aldehyde generation is shown to occur in the absence of ER stress in a model of early ALD, suggesting that ER stress may be a downstream 
consequence of oxidant burden [86]. Our data clearly demonstrate a direct mechanistic link and identify acrolein as a major pathogenic initiator of alcoholinduced hepatic injury. A causal role of acrolein in the pathogenesis of ALD is further supported by the observation that clearance of this toxic molecule by hydralazine (a known acrolein scavenger) largely prevented alcohol-induced acrolein buildup, ER stress, cell death and injury. With regard to acrolein accumulation during the development of ALD, our novel results show that alcoholmediated downregulation of GSTP may be a key mechanism that reduces the normal metabolism/clearance of acrolein, thereby contributing to hepatic accumulation of acrolein and consequent liver injury. Indeed, GSTP may be a potential therapeutic target, and pharmacological activation of GSTP may be of benefit to attenuate of ALD. Additionally, our data indicate that alcohol metabolism is essential, and that both ADH- and CYP2E1-dependent pathways contribute to acrolein accumulation. Importantly, our data show that acetaldehyde, the first metabolite of alcohol, plays a key role in alcohol-induced acrolein formation since direct exposure to acetaldehyde resulted in substantial acrolein accumulation in H4IIEC cells. Moreover, acetaldehyde may further support acrolein formation via upregulation of spermine oxidase which catalyzes the formation of free acrolein by oxidation of spermine as reported by Uemura et al. [87]. The vital role of ALDH2 in detoxification of aldehydes (both acetaldehyde and acrolein) was clearly shown by the observation that Alda1 (ALDH2 agonist) prevented alcohol-induced acrolein accumulation in cultured hepatocytes; these data are in keeping with the recent 
demonstration that Alda1 protects against alcohol-induced steatosis and cell death [88].

To the best of our knowledge our data demonstrate, for the first time, that alcohol consumption causes pathological ER stress without sufficient induction of the adaptive/protective responses involving XBP1 splicing and upregulation of ER chaperones. Thus, insufficient adaptation to ER stress along with proapoptotic signaling may significantly contribute to hepatic injury in this model of alcohol consumption. Although XBP1 splicing occurred in a few alcohol-fed mice, GRP78 or GRP94 were not significantly upregulated, suggesting that additional factors (such as other proteins or epigenetic modifications) may also be involved. A similar discordant lack of correlation has been reported in dermal fibroblasts between chaperone gene expression and upstream signal transduction (cleavage of ATF6 and splicing of XBP1) [89]. Our data in the chronic binge murine model showing minimal to no increase in chaperones GRP78 and GRP94 differs from the murine intragastric alcohol feeding model, which showed substantial upregulation of GRP78, along with CHOP and hepatic steatosis and injury [37]. Also, in guineapigs fed alcohol, liver steatosis and apoptosis were accompanied by increased mRNA and protein levels of CYP2E1 and GRP78, and activated caspase-12 [38]. These variations in ER stress-associated gene expression may reflect differences in model systems pertaining to animals, routes of alcohol feeding or temporal patterns of gene expression. Our study emphasizes the important role of pathological ER stress in alcohol-induced liver injury in this model of ALD, and indicates that impaired ER adaptive responses (e.g., GRP78) may contribute 
significantly to injury in ALD, as has been described in other forms of liver injury [90]. ER stress is known to result in JNK activation through the IRE1-TRAF pathway; however, several other stimuli also activate JNK, including TNF and TRAIL, which are known to be elevated in alcohol-fed mice. The direct causative role of ER stress in JNK activation and alcohol-induced injury is clearly suggested but not established by our work; future studies to confirm this concept will involve investigating the effects of pharmacological or genetic inhibition of ER stress on JNK activation, apoptosis and liver injury.

Notably, in addition to showing the pathogenic contribution of acrolein in the development of experimental ALD, our study provides novel and exciting evidence that acrolein removal through scavenging by hydralazine is an effective way to mitigate alcohol-induced hepatic ER stress, steatosis and injury in experimental ALD, both in vitro and in vivo. Although the protective effects of hydralazine are primarily reported against acrolein [47], it is possible that hydralazine may also interact with and neutralize other toxic aldehydes such as HNE, thereby providing added protection in ALD. In our study, hydralazine prevented liver injury when administered from the start of alcohol feeding; additional studies are needed to test its efficacy in a treatment paradigm. Although, the data convincingly demonstrate that acrolein removal/clearance by hydralazine may be a novel and effective treatment modality, the use of hydralazine in the treatment of ALD patients may be somewhat limited since hydralazine has vasodilator properties. Alternative compounds, with the same hydrazine-based acrolein scavenging mechanism but without the vasodilator effects, such as dihydralazine and the antidepressant 
phenelzine [91], may need to be investigated for safety and efficacy in ALD. Further studies are needed to understand the exact mechanism by which alcoholinduced acrolein leads to ER stress, and to examine the contribution of acrolein in alcohol-induced hepatic inflammation.

In addition to hepatic effects, alcohol-induced increased intestinal permeability and consequent systemic endotoxemia are known to be important in the pathogenesis of ALD. Alcohol is associated with intestinal barrier dysfunction and increased intestinal permeability ("leaky gut") which results in translocation of bacteria and increased systemic endotoxemia. Therefore, it becomes important to determine the role of acrolein in intestinal barrier dysfunction and the mechanism(s) by which it occurs in order to assess potential therapeutic targets for prevention and/or treatment of ALD. Our results show that acrolein adducts accumulate in the lamina propria and in the border of epithelial cells in the villi of the ileum in alcohol treated mice. Similar to hepatic data where the acrolein scavenger, hydralazine, effectively protected against alcohol-induced hepatic ER stress, steatosis and injury in experimental ALD, our intestinal data also show that hydralazine significantly reduced acrolein adduct formation in the ileum and alleviated "leaky gut" as indicated by the decrease in serum endotoxin.

These observations led us to conduct a further examination of the molecular mechanisms underlying acrolein-mediated intestinal barrier dysfunction and intestinal permeability. A well-established in vitro model of intestinal barrier function and intestinal permeability (Caco-2 cell monolayers grown on permeable inserts) were used for this study. Direct in vitro exposure of Caco-2 cells to alcohol 
resulted in acrolein adduct accumulation. Alcohol consumption is known to result in the generation of acetaldehyde in the intestine; however, it remains unclear as to whether alcohol is metabolized by gut microbiota or by intestinal epithelial (and other) cells, or both. Our data clearly indicate that alcohol is metabolized by the epithelial cells giving rise to acrolein. Also, our data indicate that alcohol-induced acrolein adduct accumulation in the intestinal epithelial cells is a driving force to cause barrier dysfunction and leaky gut. Our in vitro data indicate that acrolein mimics the effect of alcohol in the epithelial cells and alters the localization and redistribution of tight junction proteins, hence causes the disruption of intestinal barrier function and enhances intestinal permeability. The similarity of the results observed with acrolein compared to alcohol provide further confirmation that alcohol-induced acrolein disrupts intestinal barrier function and leads to the increased intestinal permeability and further contributes to the pathogenesis of ALD.

In conclusion, acrolein is the most reactive and toxic aldehyde generated through lipid peroxidation. Accumulation of acrolein adducts was seen in response to alcohol consumption in mouse livers and intestines. Acrolein adduct accumulation correlated with disruption of TJPs, intestinal barrier dysfunction, and intestinal permeability in the gut. Moreover, accumulation of acrolein adducts is associated with hepatic steatosis, JNK activation, ER stress, apoptosis and liver injury, which are recognized etiologic factors in gut-liver injury of ALD. We used cultured hepatic and intestinal cells to examine the in vitro effects of acrolein compared to alcohol. Alcohol-induced in vivo intestinal effects were mimicked by 
acrolein in vitro Caco-2 cells, with downregulation of tight junction proteins, disruption of TEER, and increased FD-4 permeability. Similar to alcohol, in vitro acrolein triggered hepatocyte ER stress and induced apoptosis. Together the data indicate that acrolein may mediate the adverse intestinal and hepatic effects of alcohol. Notably, these effects were attenuated by the acrolein scavenger, hydralazine, suggesting its therapeutic potential in the gut-liver injury of ALD.

Limitation of the study: The concentration of free acrolein is difficult to measure in biological samples due to its high reactivity and lability. In this study, we used an indirect measure of acrolein and only assessed the levels of FDPlysine acrolein adducts, and no other adducts that may be formed with cysteines and histidines. The development of more efficient novel assays for free acrolein and adducts would facilitate more accurate measurement of acrolein in ALD. In the intestinal barrier function study, we focused on the TJPs. However, the mucus layer (mucins and intestinal trefoil factors) [90] and/or antimicrobial peptides also critically contribute to gut integrity and are affected by alcohol. These will be highly relevant studies as a future direction for the project.

Currently, there are several models of ALD that are similar in many aspects, but also differ somewhat. Moreover, no animal model perfectly simulates human ALD. Our data are limited to one single model of chronic binge. To emphasize the relevance of our findings, the reproducibility across animal models would need to be further examined. Moreover, mouse data may not exactly correlate to the responses in human ALD; hence, the role of acrolein in human ALD would need to be investigated and established. 
Clinical relevance: About $30 \%$ of heavy alcohol drinkers develop severe ALD, and dietary and environmental factors are thought to be critical determinants. Acrolein is a common environmental and dietary pollutant and our study shows that it is a major mediator of the adverse effects of alcohol and plays a causal role in ALD pathogenesis. Our group has shown that diets enriched in linoleic acid (common dietary PUFA) exacerbate alcohol-induced liver injury [83], and consumption of linoleic acid has increased more than 3-fold over the last century, thereby increasing the substrate availability for LPO and subsequent acrolein generation. Additionally, acrolein is a major aldehyde component of cigarette smoke [92]; cigarette smoking is common in persons consuming alcohol and is known to negatively impact alcoholic liver disease. Thus, environmental and dietary acrolein exposures may add to endogenously generated acrolein with significant and clinically relevant pathogenic consequences. Our results indicate that acrolein is a major pathogenic contributor and may be an important therapeutic target in ALD. Notably, our study demonstrates for the first time that acrolein neutralization/clearance may be an effective strategy for curtailing alcohol-induced acrolein-mediated gut-liver injury in the development of ALD. Hence, acrolein scavengers such as hydralazine may represent a novel therapeutic approach for the prevention/treatment of ALD. 


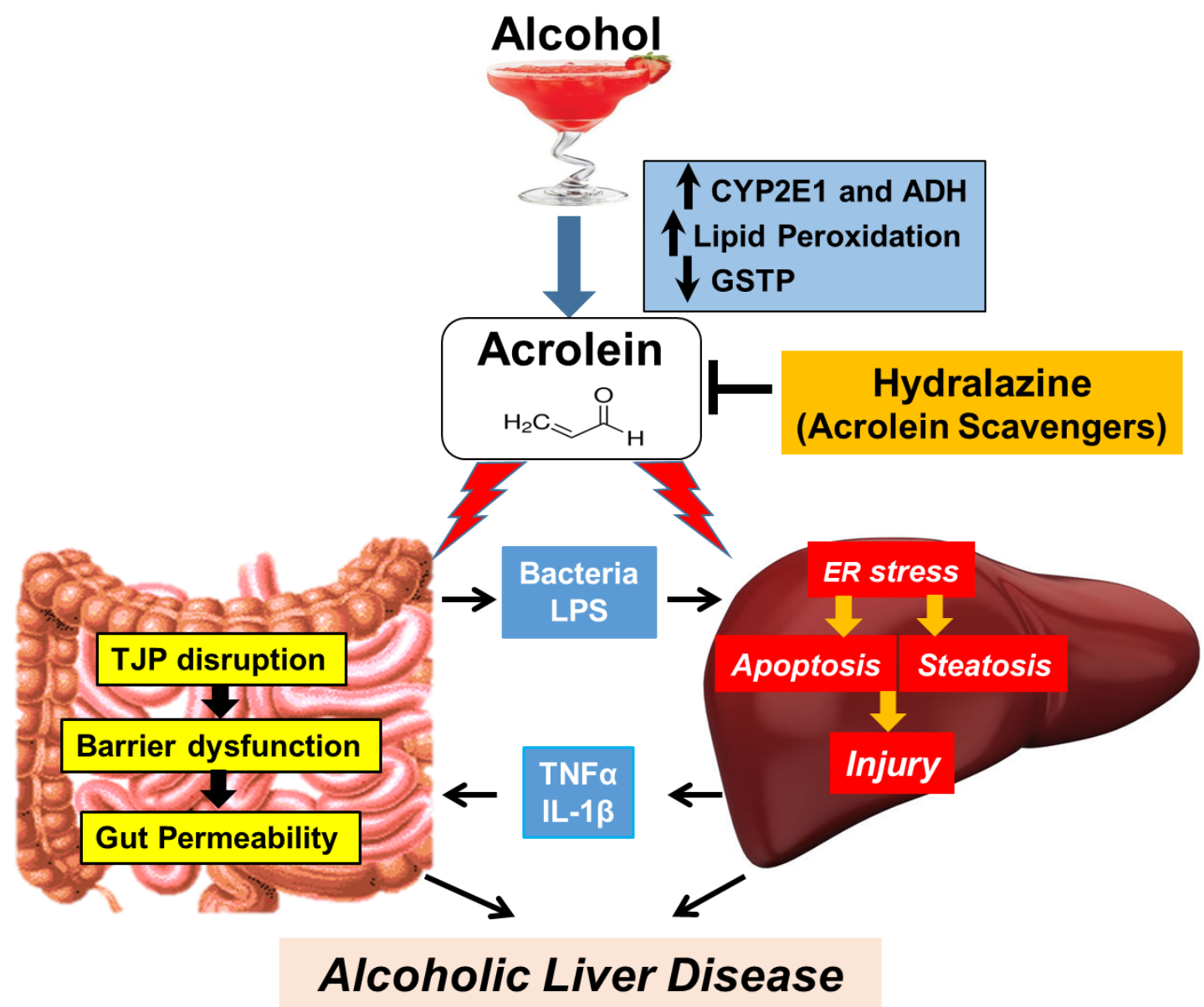

Figure 25. Schematic: Acrolein mediates alcohol-induced intestinal barrier dysfunction, permeability and hepatic ER stress, apoptosis and injury in ALD, and the scavenger, hydralazine, prevents these effects. 


\section{REFERENCES}

1. Rehm, J., A.V. Samokhvalov, and K.D. Shield, Global burden of alcoholic liver diseases. J Hepatol, 2013. 59(1): p. 160-8.

2. Louvet, A. and P. Mathurin, Alcoholic liver disease: mechanisms of injury and targeted treatment. Nat Rev Gastroenterol Hepatol, 2015. 12(4): p. 231-242.

3. French, S.W., The mechanism of organ injury in alcoholics: implications for therapy. Alcohol Alcohol Suppl, 1991. 1: p. 57-63.

4. Crabb, D.W., Pathogenesis of alcoholic liver disease: newer mechanisms of injury. Keio J Med, 1999. 48(4): p. 184-8.

5. NIAAA: Celebrating 40 Years of Alcohol Research. 2010. 33(No. 1\&2).

6. Ji, C., Mechanisms of alcohol-induced endoplasmic reticulum stress and organ injuries. Biochem Res Int, 2012. 2012: p. 216450.

7. Ambrozewicz, E., et al., Black-currant protection against oxidative stress formation. J Toxicol Environ Health A, 2013. 76(23): p. 1293-306.

8. Cederbaum, A.I., Y. Lu, and D. Wu, Role of oxidative stress in alcoholinduced liver injury. Arch Toxicol, 2009. 83(6): p. 519-48.

9. Rett, B.S. and J. Whelan, Increasing dietary linoleic acid does not increase tissue arachidonic acid content in adults consuming Westerntype diets: a systematic review. Nutr Metab (Lond), 2011. 8: p. 36. 
10. Kirpich, I., et al., Binge ethanol-induced HDAC3 down-regulates Cpt1alpha expression leading to hepatic steatosis and injury. Alcohol Clin Exp Res, 2013. 37(11): p. 1920-9.

11. Calder, P.C., n-3 polyunsaturated fatty acids, inflammation, and inflammatory diseases. Am J Clin Nutr, 2006. 83(6 Suppl): p. 1505S$1519 S$.

12. Smathers, R.L., et al., Overview of lipid peroxidation products and hepatic protein modification in alcoholic liver disease. Chem Biol Interact, 2011. 192(1-2): p. 107-12.

13. Keshavarzian, A., et al., Evidence that chronic alcohol exposure promotes intestinal oxidative stress, intestinal hyperpermeability and endotoxemia prior to development of alcoholic steatohepatitis in rats. J Hepatol, 2009. 50(3): p. 538-47.

14. Pizzimenti, S., et al., Interaction of aldehydes derived from lipid peroxidation and membrane proteins. Front Physiol, 2013. 4: p. 242.

15. Ohno, Y. and K. Ormstad, Formation, toxicity and inactivation of acrolein during biotransformation of cyclophosphamide as studied in freshly isolated cells from rat liver and kidney. Arch Toxicol, 1985. 57(2): p. 99103.

16. Lee, S.E. and Y.S. Park, Role of lipid peroxidation-derived alpha, betaunsaturated aldehydes in vascular dysfunction. Oxid Med Cell Longev, 2013. 2013: p. 629028. 
17. Tully, M., L. Zheng, and R. Shi, Acrolein detection: potential theranostic utility in multiple sclerosis and spinal cord injury. Expert Rev Neurother, 2014. 14(6): p. 679-85.

18. Stevens, J.F. and C.S. Maier, Acrolein: sources, metabolism, and biomolecular interactions relevant to human health and disease. Mol Nutr Food Res, 2008. 52(1): p. 7-25.

19. Huang, Y.J., et al., Acrolein induces Alzheimer's disease-like pathologies in vitro and in vivo. Toxicol Lett, 2013. 217(3): p. 184-91.

20. Daimon, M., et al., Increased urinary levels of pentosidine, pyrraline and acrolein adduct in type 2 diabetes. Endocr J, 2003. 50(1): p. 61-7.

21. Shamoto-Nagai, M., et al., In parkinsonian substantia nigra, alphasynuclein is modified by acrolein, a lipid-peroxidation product, and accumulates in the dopamine neurons with inhibition of proteasome activity. J Neural Transm, 2007. 114(12): p. 1559-67.

22. Park, Y.S. and N. Taniguchi, Acrolein induces inflammatory response underlying endothelial dysfunction: a risk factor for atherosclerosis. Ann $\mathrm{N}$ Y Acad Sci, 2008. 1126: p. 185-9.

23. Moghe, A., et al., Molecular mechanisms of acrolein toxicity: relevance to human disease. Toxicol Sci, 2015. 143(2): p. 242-55.

24. Kitaguchi, Y., et al., Acrolein induces endoplasmic reticulum stress and causes airspace enlargement. PLoS One, 2012. 7(5): p. e38038.

25. McClain, C.J., et al., Cytokines in alcoholic liver disease. Semin Liver Dis, 1999. 19(2): p. 205-19. 
26. Compare, D., et al., Gut--liver axis: the impact of gut microbiota on non alcoholic fatty liver disease. Nutr Metab Cardiovasc Dis, 2012. 22(6): p. $471-6$.

27. Bailey, S.M., A review of the role of reactive oxygen and nitrogen species in alcohol-induced mitochondrial dysfunction. Free Radic Res, 2003. 37(6): p. 585-96.

28. Knockaert, L., B. Fromenty, and M.A. Robin, Mechanisms of mitochondrial targeting of cytochrome P450 2E1: physiopathological role in liver injury and obesity. FEBS J, 2011. 278(22): p. 4252-60.

29. van Meer, G., D.R. Voelker, and G.W. Feigenson, Membrane lipids: where they are and how they behave. Nat Rev Mol Cell Biol, 2008. 9(2): p. 11224.

30. Ozcan, L. and I. Tabas, Role of endoplasmic reticulum stress in metabolic disease and other disorders. Annu Rev Med, 2012. 63: p. 317-28.

31. Cao, S.S. and R.J. Kaufman, Unfolded protein response. Curr Biol, 2012. 22(16): p. R622-6.

32. Verma, G. and M. Datta, The critical role of JNK in the ER-mitochondrial crosstalk during apoptotic cell death. J Cell Physiol, 2012. 227(5): p. 17915.

33. Yoshida, H., ER stress and diseases. FEBS J, 2007. 274(3): p. 630-58.

34. Malhi, H. and R.J. Kaufman, Endoplasmic reticulum stress in liver disease. J Hepatol, 2011. 54(4): p. 795-809. 
35. Magne, L., et al., ATF4 and the integrated stress response are induced by ethanol and cytochrome P450 2E1 in human hepatocytes. J Hepatol, 2011. 54(4): p. 729-37.

36. Ji, C. and N. Kaplowitz, Betaine decreases hyperhomocysteinemia, endoplasmic reticulum stress, and liver injury in alcohol-fed mice. Gastroenterology, 2003. 124(5): p. 1488-99.

37. Ji, C., et al., Role of CHOP in hepatic apoptosis in the murine model of intragastric ethanol feeding. Alcohol Clin Exp Res, 2005. 29(8): p. 1496503.

38. Esfandiari, F., et al., Chronic ethanol feeding and folate deficiency activate hepatic endoplasmic reticulum stress pathway in micropigs. Am J Physiol Gastrointest Liver Physiol, 2005. 289(1): p. G54-63.

39. Ji, C. and N. Kaplowitz, ER stress: can the liver cope? J Hepatol, 2006. 45(2): p. 321-33.

40. Ji, C., New Insights into the Pathogenesis of Alcohol-Induced ER Stress and Liver Diseases. Int J Hepatol, 2014. 2014: p. 513787.

41. Shah, H., et al., Protection of HepG2 cells against acrolein toxicity by 2cyano-3,12-dioxooleana-1,9-dien-28-imidazolide via glutathione-mediated mechanism. Exp Biol Med (Maywood), 2015. 240(10): p. 1340-51.

42. Beauchamp, R.O., Jr., et al., A critical review of the literature on acrolein toxicity. Crit Rev Toxicol, 1985. 14(4): p. 309-80. 
43. Parent, R.A., et al., Metabolism and distribution of [2,3-14C]acrolein in Sprague-Dawley rats. II. Identification of urinary and fecal metabolites. Toxicol Sci, 1998. 43(2): p. 110-20.

44. Berhane, K., et al., Detoxication of base propenals and other alpha, betaunsaturated aldehyde products of radical reactions and lipid peroxidation by human glutathione transferases. Proc Natl Acad Sci U S A, 1994. 91(4): p. $1480-4$.

45. Uemura, T., et al., Aggravation of brain infarction through an increase in acrolein production and a decrease in glutathione with aging. Biochem Biophys Res Commun, 2016. 473(2): p. 630-5.

46. Park, J., B. Muratori, and R. Shi, Acrolein as a novel therapeutic target for motor and sensory deficits in spinal cord injury. Neural Regen Res, 2014. 9(7): p. 677-83.

47. Burcham, P.C. and S.M. Pyke, Hydralazine inhibits rapid acrolein-induced protein oligomerization: role of aldehyde scavenging and adduct trapping in cross-link blocking and cytoprotection. Mol Pharmacol, 2006. 69(3): p. 1056-65.

48. Hipkiss, A.R. and H. Chana, Carnosine protects proteins against methylglyoxal-mediated modifications. Biochem Biophys Res Commun, 1998. 248(1): p. 28-32.

49. Szabo, G. and S. Bala, Alcoholic liver disease and the gut-liver axis. World J Gastroenterol, 2010. 16(11): p. 1321-9. 
50. Catalioto, R.M., C.A. Maggi, and S. Giuliani, Intestinal epithelial barrier dysfunction in disease and possible therapeutical interventions. Curr Med Chem, 2011. 18(3): p. 398-426.

51. Catanzaro, R., et al., The gut microbiota and its correlations with the central nervous system disorders. Panminerva Med, 2015. 57(3): p. 12743.

52. Szabo, G., Gut-liver axis in alcoholic liver disease. Gastroenterology, 2015. 148(1): p. 30-6.

53. Bischoff, S.C., et al., Intestinal permeability--a new target for disease prevention and therapy. BMC Gastroenterol, 2014. 14: p. 189.

54. Kucharzik, T., et al., Neutrophil transmigration in inflammatory bowel disease is associated with differential expression of epithelial intercellular junction proteins. Am J Pathol, 2001. 159(6): p. 2001-9.

55. Montalto, M., et al., Immunohistochemical analysis of ZO-1 in the duodenal mucosa of patients with untreated and treated celiac disease. Digestion, 2002. 65(4): p. 227-33.

56. Groschwitz, K.R. and S.P. Hogan, Intestinal barrier function: molecular regulation and disease pathogenesis. J Allergy Clin Immunol, 2009. 124(1): p. 3-20; quiz 21-2.

57. Robinson, K., et al., Regulation of the Intestinal Barrier Function by Host Defense Peptides. Front Vet Sci, 2015. 2: p. 57. 
58. Pereira, C., et al., Dissecting stromal-epithelial interactions in a 3D in vitro cellularized intestinal model for permeability studies. Biomaterials, 2015. 56: p. $36-45$.

59. Diamond, G., et al., The roles of antimicrobial peptides in innate host defense. Curr Pharm Des, 2009. 15(21): p. 2377-92.

60. Zhong, W., et al., The role of zinc deficiency in alcohol-induced intestinal barrier dysfunction. Am J Physiol Gastrointest Liver Physiol, 2010. 298(5): p. G625-33.

61. Suzuki, T., Regulation of intestinal epithelial permeability by tight junctions. Cell Mol Life Sci, 2013. 70(4): p. 631-59.

62. Gunzel, D. and A.S. Yu, Claudins and the modulation of tight junction permeability. Physiol Rev, 2013. 93(2): p. 525-69.

63. Herve, J.C., et al., Influence of the scaffolding protein Zonula Occludens (ZOs) on membrane channels. Biochim Biophys Acta, 2014. 1838(2): p. 595-604.

64. Luissint, A.C., A. Nusrat, and C.A. Parkos, JAM-related proteins in mucosal homeostasis and inflammation. Semin Immunopathol, 2014. 36(2): p. 211-26.

65. Price, D., L. Ackland, and C. Suphioglu, Nuts ' $n$ ' guts: transport of food allergens across the intestinal epithelium. Asia Pac Allergy, 2013. 3(4): p. $257-65$. 
66. Sambuy, Y., et al., The Caco-2 cell line as a model of the intestinal barrier: influence of cell and culture-related factors on Caco-2 cell functional characteristics. Cell Biol Toxicol, 2005. 21(1): p. 1-26.

67. Mathews, S., et al., Animals models of gastrointestinal and liver diseases. Animal models of alcohol-induced liver disease: pathophysiology, translational relevance, and challenges. Am J Physiol Gastrointest Liver Physiol, 2014. 306(10): p. G819-23.

68. Bertola, A., et al., Mouse model of chronic and binge ethanol feeding (the NIAAA model). Nat Protoc, 2013. 8(3): p. 627-37.

69. Roberts, B.J., et al., Ethanol induces CYP2E1 by protein stabilization. Role of ubiquitin conjugation in the rapid degradation of CYP2E1. J Biol Chem, 1995. 270(50): p. 29632-5.

70. Feierman, D.E. and A.I. Cederbaum, Inhibition of microsomal oxidation of ethanol by pyrazole and 4-methylpyrazole in vitro. Increased effectiveness after induction by pyrazole and 4-methylpyrazole. Biochem J, 1986. 239(3): p. 671-7.

71. Sovolyova, N., et al., Stressed to death - mechanisms of ER stressinduced cell death. Biol Chem, 2014. 395(1): p. 1-13.

72. Seki, E., D.A. Brenner, and M. Karin, A liver full of JNK: signaling in regulation of cell function and disease pathogenesis, and clinical approaches. Gastroenterology, 2012. 143(2): p. 307-20. 
73. Rao, R.V., et al., Coupling endoplasmic reticulum stress to the cell death program. An Apaf-1-independent intrinsic pathway. J Biol Chem, 2002. 277(24): p. 21836-42.

74. Sano, R. and J.C. Reed, ER stress-induced cell death mechanisms. Biochim Biophys Acta, 2013. 1833(12): p. 3460-70.

75. Logue, S.E., et al., New directions in ER stress-induced cell death. Apoptosis, 2013. 18(5): p. 537-46.

76. Henkel, A. and R.M. Green, The unfolded protein response in fatty liver disease. Semin Liver Dis, 2013. 33(4): p. 321-9.

77. Hamann, K. and R. Shi, Acrolein scavenging: a potential novel mechanism of attenuating oxidative stress following spinal cord injury. J Neurochem, 2009. 111(6): p. 1348-56.

78. Burcham, P.C., P.G. Kerr, and F. Fontaine, The antihypertensive hydralazine is an efficient scavenger of acrolein. Redox Rep, 2000. 5(1): p. 47-9.

79. Kaminskas, L.M., S.M. Pyke, and P.C. Burcham, Strong protein adduct trapping accompanies abolition of acrolein-mediated hepatotoxicity by hydralazine in mice. J Pharmacol Exp Ther, 2004. 310(3): p. 1003-10.

80. Kaminskas, L.M., S.M. Pyke, and P.C. Burcham, Reactivity of hydrazinophthalazine drugs with the lipid peroxidation products acrolein and crotonaldehyde. Org Biomol Chem, 2004. 2(18): p. 2578-84. 
81. Park, J., et al., Neuroprotective role of hydralazine in rat spinal cord injuryattenuation of acrolein-mediated damage. J Neurochem, 2014. 129(2): p. $339-49$.

82. Yan, S.L., et al., Protective effects from carnosine and histidine on acetaminophen-induced liver injury. J Food Sci, 2009. 74(8): p. H259-65.

83. Kirpich, I.A., et al., The type of dietary fat modulates intestinal tight junction integrity, gut permeability, and hepatic toll-like receptor expression in a mouse model of alcoholic liver disease. Alcohol Clin Exp Res, 2012. 36(5): p. 835-46.

84. Tian, S., et al., Curcumin protects against the intestinal ischemiareperfusion injury: involvement of the tight junction protein ZO-1 and TNFalpha related mechanism. Korean J Physiol Pharmacol, 2016. 20(2): p. $147-52$.

85. Zhou, Q., et al., MicroRNA 29 targets nuclear factor-kappaB-repressing factor and Claudin 1 to increase intestinal permeability. Gastroenterology, 2015. 148(1): p. 158-169 e8.

86. Galligan, J.J., et al., Oxidative Stress and the ER Stress Response in a Murine Model for Early-Stage Alcoholic Liver Disease. J Toxicol, 2012. 2012: p. 207594.

87. Uemura, T., et al., Acetaldehyde-induced cytotoxicity involves induction of spermine oxidase at the transcriptional level. Toxicology, 2013. 310: p. 1-7. 
88. Zhong, W., et al., Pharmacological activation of aldehyde dehydrogenase 2 by Alda- 1 reverses alcohol-induced hepatic steatosis and cell death in mice. J Hepatol, 2014.

89. Shang, J. and M.A. Lehrman, Discordance of UPR signaling by ATF6 and Ire1p-XBP1 with levels of target transcripts. Biochem Biophys Res Commun, 2004. 317(2): p. 390-6.

90. Ji, C., et al., Liver-specific loss of glucose-regulated protein 78 perturbs the unfolded protein response and exacerbates a spectrum of liver diseases in mice. Hepatology, 2011. 54(1): p. 229-39.

91. Zheng, L., et al., Determination of urine 3-HPMA, a stable acrolein metabolite in a rat model of spinal cord injury. J Neurotrauma, 2013. 30(15): p. 1334-41.

92. Smith, C.J. and T.H. Fischer, Particulate and vapor phase constituents of cigarette mainstream smoke and risk of myocardial infarction. Atherosclerosis, 2001. 158(2): p. 257-67. 


\author{
CURRICULUM VITAE \\ Name: Wei Yang (Jeremy), Chen \\ Address: 1501 Shelburne Circle, Unit 212, Louisville, KY, 40208 \\ Cell phone: 502-7445753 \\ Email:w0chen07@louisville.edu
}

Date of Birth : December 22, 1980

Birth Place : Taipei, Taiwan

Education

2014-2016

2010-2014

2007-2009

1999-2003

Positions Held

2010-2016

$2010-2010$

2007-2009

2004-2007

2003-2003
Doctor of Philosophy, Pharmacology \& Toxicology, University of Louisville, KY

Master of Science, Pharmacology and Toxicology, University of Louisville, KY

Thesis: Pathogenic role of acrolein in alcoholic liver disease

Master of Science in Biochemistry, University of North Carolina-Greensboro, NC

Thesis: The inhibition of human cytochrome P4502E1 by essential oil of Lemongrass and the primary aldehyde constituent of lemongrass, citral

Bachelor of Science in Chemistry, Tamkang University, Taipei, Taiwan

Graduate Student (PhD), University of Louisville, Louisville, KY

Research Assistant, Kaohsiung Medical University, Kaohsiung, Taiwan

Teaching Assistant, University of North CarolinaGreensboro, Greensboro, NC

Environmental Technician, SGS Group - Société Générale de Surveillance, Taipei, Taiwan

Infantry, Army of Taiwan, Taiwan

\title{
Awards and Honors
}

2016

2015

2015
SOT Graduate Student Travel Support, Society of Toxicology (SOT), New Orleans, LA Presidential Poster of Distinction, American Association for the Study of Liver Diseases (AASLD), San Francisco, CA RSA Student Merit Award, Research Society for Alcoholism (RSA), San Antonio, TX 
2015

2014

2014

2014-2015

2014

2013

2012-2013

2010-2012
Research Committee Travel Award for SOT 2015, University of Louisville

$1^{\text {st }}$ Place Student Poster Award, NIEHS Tamburro

Environmental Liver Symposium, Louisville, KY

Graduate School Council Travel Award for DDW 2014, University of Louisville

Sponsored Research Tuition Award, University of Louisville

UofL School of Medicine Travel Award for SOT 2014, University of Louisville

Graduate School Council Travel Award for AASLD 2013, University of Louisville

Sponsored Research Tuition Award, University of Louisville IPIBS Integrated Programs in Biomedical Sciences (IPIBS)

fellowship award, University of Louisville

\section{Professional Society Memberships}

The American Society for Pharmacology and Experimental Therapeutics

Society of Toxicology

Ohio Valley Society of Toxicology

\section{Peer-reviewed publications}

1. Wei-Yang Chen, Jingwen Zhang, Smita S. Ghare, Shirish Barve, Craig McClain, and Swati Joshi-Barve. Acrolein, a lipid-derived aldehyde, is a critical pathogenic mediator and potential therapeutic target for alcoholic liver disease.

(Cellular and Molecular Gastroenterology and Hepatology).

2. Smita S. Ghare, Hridgandh Donde, Wei-Yang Chen, David F. Barker, Leila Gobejishvilli, Craig J. McClain, Shirish S. Barve, and Swati Joshi-Barve.

Acrolein enhances epigenetic modifications, FasL expression and hepatocyte toxicity induced by anti-HIV drug Zidovudine.

(Toxicology in Vitro).

3. Wei-Yang Chen, Jingwen Zhang, Shirish Barve, Craig McClain, and Swati JoshiBarve. The role of Acrolein in the intestinal barrier dysfunction in alcoholic liver disease. (Manuscript under preparation).

\section{Abstracts}

1. Society of Toxicology (SOT) 2016. New Orleans, LA, March 13-17, 2016.

Wei-Yang Chen, Jingwen Zhang, Craig McClain, Shirish Barve, and Swati JoshiBarve. Acrolein, a lipid-derived aldehyde, is a pathogenic mediator of gut-liver injury in alcoholic liver disease. SOT Graduate Student Travel Support

2. AASLD 2015. The Liver meeting. San Francisco, CA, November 12-17, 2015. Wei-Yang Chen, Jingwen Zhang, Craig McClain, Shirish Barve, and Swati JoshiBarve. Acrolein, a lipid-derived aldehyde, is a critical pathogenic mediator and potential therapeutic target for alcoholic liver disease. Presidential Poster of Distinction

3. Research Society for Alcoholism (RSA) 2015 Annual Meeting, San Antonio, TX, June 20-24, 2015.

Wei-Yang Chen, Jingwen Zhang, Craig McClain, Shirish Barve, and Swati JoshiBarve. Lipid-derived aldehyde, acrolein, is a critical mediator of alcohol-induced 
gut-liver injury in alcoholic liver disease. RSA Student Merit Award

4. American Society for Pharmacology and Experimental Therapeutics (ASPET)

2015. Boston, MA, March 28- April 1, 2015.

Wei-Yang Chen, Jingwen Zhang, Craig McClain, Shirish Barve, and Swati JoshiBarve. Lipid-derived aldehyde, acrolein, is a critical mediator of alcohol-induced gut-liver injury in alcoholic liver disease.

5. Society of Toxicology (SOT) 2015. San Diego, CA, March 22-26, 2015.

Wei-Yang Chen, Jingwen Zhang, Craig McClain, Shirish Barve, and Swati JoshiBarve. Lipid-derived aldehyde, acrolein, is a critical mediator of alcohol-induced gut-liver injury in alcoholic liver disease. Research Committee Travel Award for SOT 2015

6. AASLD 2014 The Liver meeting. Boston, MA, November 7-11, 2014.

Wei-Yang Chen, Jingwen Zhang, Craig McClain, Shirish Barve, and Swati JoshiBarve. Lipid-derived aldehyde, acrolein, is a critical mediator of alcohol-induced gut-liver injury in alcoholic liver disease.

7. OVSOT (Ohio Valley Society of Toxicology) 2014 Annual Meeting. Dayton, OH, September 26, 2014.

Wei-Yang Chen, Jingwen Zhang, Craig McClain, Shirish Barve, and Swati JoshiBarve. Lipid-derived aldehyde, acrolein, is a critical mediator of alcohol-induced gut-liver injury in alcoholic liver disease.

8. Research! Louisville 2014. Louisville, KY, September 16-19, 2014.

Wei-Yang Chen, Jingwen Zhang, Craig McClain, Shirish Barve, and Swati JoshiBarve. Lipid-derived aldehyde, acrolein, is a critical mediator of alcohol-induced gut-liver injury in alcoholic liver disease.

9. NIEHS Tamburro Environmental Liver Symposium. Louisville, KY, September 11-12, 2014.

Wei-Yang Chen, Jingwen Zhang, Craig McClain, Shirish Barve, and Swati JoshiBarve. Lipid-derived aldehyde, acrolein, is a critical mediator of Alcohol- induced gut-liver injury in alcoholic liver disease. $1^{\text {st }}$ Place Student Poster Award

10. American Society for Pharmacology and Experimental Therapeutics (ASPET) 2014. San Diego, CA, April 26-30, 2014.

Wei-Yang Chen, Jingwen Zhang, Craig McClain, Shirish Barve, and Swati JoshiBarve. Lipid-derived aldehyde, acrolein, is a critical mediator of alcohol-induced gut-liver injury.

11. Society of Toxicology (SOT) 2014. Phoenix, AZ, March 24-27, 2014.

Wei-Yang Chen, Jingwen Zhang, Craig McClain, Shirish Barve, and Swati JoshiBarve. Acrolein, a Lipid-Derived Aldehyde Metabolite, Is a Critical Mediator of Alcohol-Induced Endoplasmic Reticulum Stress and Liver Injury. UofL School of Medicine Travel Award for SOT 2014

12. AASLD 2013 The Liver meeting. Washington, D.C., November 1-5, 2013. Wei-Yang Chen, Jingwen Zhang, Craig McClain, Shirish Barve, and Swati JoshiBarve. Acrolein, a reactive aldehyde metabolite, is a major mediator of alcoholinduced endoplasmic reticulum stress and liver injury. UofL Graduate Student Travel Award for AASLD 2013

13. Research! Louisville 2013, Louisville, KY, 2013.

Wei-Yang Chen, Jingwen Zhang, Craig McClain, Shirish Barve, and Swati JoshiBarve. Acrolein, a reactive aldehyde metabolite, is a major mediator of alcoholinduced endoplasmic reticulum stress and liver injury.

14. OVSOT (Ohio Valley Society of Toxicology) 2013 Annual Meeting. Louisville, KY, September 13, 2013. 
Wei-Yang Chen, Jingwen Zhang, Craig McClain, Shirish Barve, and Swati JoshiBarve. Acrolein, a reactive aldehyde metabolite, is a major mediator of alcoholinduced endoplasmic reticulum stress and liver injury.

15. Research Society for Alcoholism (RSA) 2013 Annual Meeting, Orlando, FL, June 22-26, 2013.

Wei-Yang Chen, Shirish Barve, Jingwen Zhang, Mohammad Mohammad, Craig McClain, Swati Joshi-Barve. Binge alcohol impairs IFNa antiviral gene expression in mice. Research Committee Travel Award for RSA 2013

16. OVSOT (Ohio Valley Society of Toxicology) 2012 Annual Meeting, Columbus, $\mathrm{OH}$, September 28, 2012.

Wei-Yang Chen, Smita Ghare, Hridgandh Donde, Swati Joshi-Barve, Shirish Barve, Craig McClain.

The role of neutrophil chemotactic cytokine - interleukin 8 in HAART-induced hepatotoxicity.

17. OVSOT (Ohio Valley Society of Toxicology) 2012 Annual Meeting, Columbus, $\mathrm{OH}$, September 28, 2012.

Wei-Yang Chen, Smita Ghare, Hridgandh Donde, Swati Joshi-Barve, Shirish Barve, Craig McClain.

The role of neutrophil chemotactic cytokine - interleukin 8 in HAART-induced hepatotoxicity.

18. Research! Louisville, Louisville, KY, 2012

Wei-Yang Chen, Smita Ghare, Hridgandh Donde, Swati Joshi-Barve, Shirish Barve, Craig McClain.

The role of neutrophil chemotactic cytokine - interleukin 8 in HAART-induced hepatotoxicity.

19. Research! Louisville 2011, Louisville, KY, 2011.

Wei-Yang Chen, Smita Ghare, Hridgandh Donde, Swati Joshi-Barve, Craig McClain and Shirish Barve. Induction of the neutrophil chemotactic cytokine interleukin8 (IL-8) by HAART (Highly Active Anti-Retroviral Therapy): Relevance to HAART induced hepatotoxicity,

20. SERMACS 2008 (Southeastern Regional Meeting of the American Chemical Society), Nashville, TN, November, 12-15.

Wei-Yang Chen, Jill Sollenberger, Katherine Ryan, Tamoor Asif, Gregory M. Raner. Inhibition of Human Cytochrome P4502E1 by Aldehyde-Containing Essential Oils.

\section{Lecture presentation}

2014

2014
Digestive Disease Week (DDW) 2014

Chicago, IL

Lipid-derived aldehyde, acrolein, is a critical mediator of alcohol-induced gut-liver injury

OVSOT (Ohio Valley Society of Toxicology) Summer Student Meeting Louisville, KY

Lipid-derived aldehyde, acrolein, is a critical mediator of alcohol-induced gut-liver injury 